University of Redlands

\title{
Promoting the Protection of Marine Biogeographic Regions
}

A Major Individual Project submitted in partial satisfaction of the requirements for the degree of Master of Science in Geographic Information Systems

\author{
by \\ Qian Song
}

Ruijin Ma, Ph.D., Committee Chair

Nader Afzalan, Ph.D.

September 2016 
Promoting the Protection of Marine Biogeographic Regions

Copyright (C) 2016

by

Qian Song 
The report of Qian Song is approved.
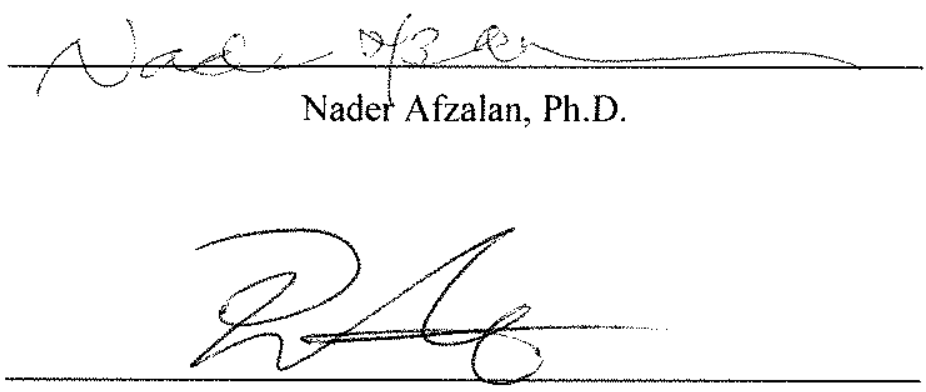

Ruijin Ma, Ph.D., Committee Chair

September 2016 



\section{Acknowledgements}

Special acknowledgments to my advisor, Dr. Ruijin Ma, who helped me complete the project smoothly. I am grateful for his suggestions and guidance throughout the project. I also would like to thank Dr. Nader Afzalan who took the time to be my second reader. Thank you to all the MS GIS program faculty, you provided assistance and guidance throughout the whole program. Thanks to Andrea Barrios. It's lucky that we have you as the program coordinator to ensure the smooth running of the program. Thanks to Ruben Ortiz. Without your help to setup a GIS server and web server for me, it would have been impossible to deliver this project. Thanks to Lynn Flewelling for spending time on editing the paper.

I want to thank my classmates in Cohort 28 for their support and friendship. You helped me through all the tough times and good times. I really enjoyed learning and working with you together.

Last but not the least, a big hug and thanks to my parents. During all the highs and lows in my life, you always believe in and encourage me. Without your support, I could not attend and finish this program. 



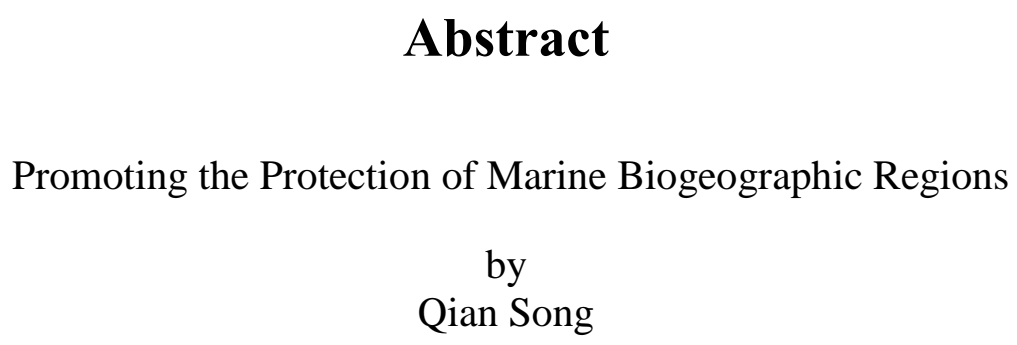

Losses in marine biodiversity are becoming increasingly severe. In order to safeguard marine biodiversity, the Marine Conservation Institute (MCI) wanted a streamlined interface for use in proposing new marine protected areas (MPAs) and making the protection more consistent among marine biogeographic regions (MBRs). The institute also needed a new way to collaborate with its partners to propose new MPAs. The project provided a useful tool - an interactive web GIS application developed using Hypertext Markup Language, Cascading Style Sheets, and JavaScript—-for MCI and its partners according to their needs. A geoprocessing service was consumed in the web application to calculate the protection coverage among MBRs. The web application contains four panels to help users share the information about MPAs and MBRs, design a new MPA graphically, and then update the MPA. It gives MPA designers an overview of the current and future protection status of each MBR and helps them propose new MPAs wisely and effectively. 



\section{Table of Contents}

Chapter 1 - Introduction ................................................................................................ 1

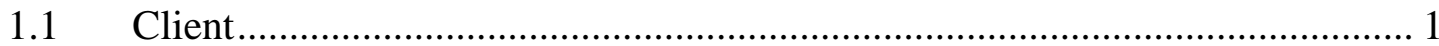

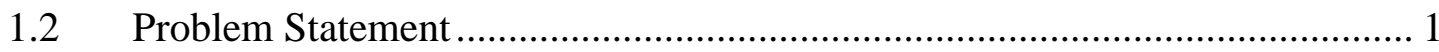

$1.3 \quad$ Proposed Solution ............................................................................... 1

1.3.1 Goals and Objectives ........................................................................... 2

1.3.2 Scope

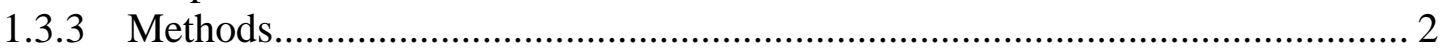

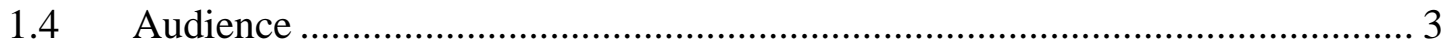

1.5 Overview of the Rest of this Report ........................................................... 3

Chapter 2 - Background and Literature Review ............................................................. 5

$2.1 \quad$ Marine Biogeographic Regions …………………................................. 5

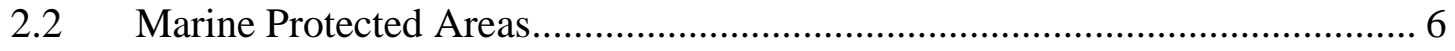

2.3 GIS Application for Marine Protection....................................................... 7

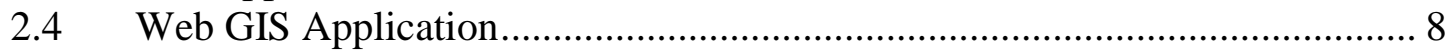

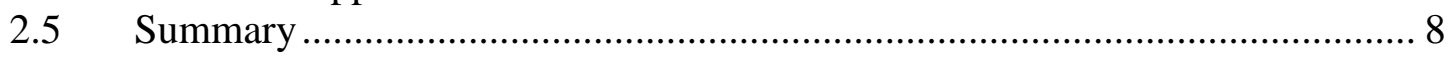

Chapter 3 - Systems Analysis and Design....................................................................... 9

$3.1 \quad$ Problem Statement .......................................................................... 9

3.2 Requirements Analysis ........................................................................... 9

3.2.1 Functional requirements...................................................................... 9

3.2.2 Non-functional requirements ................................................................... 10

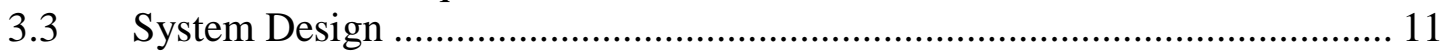

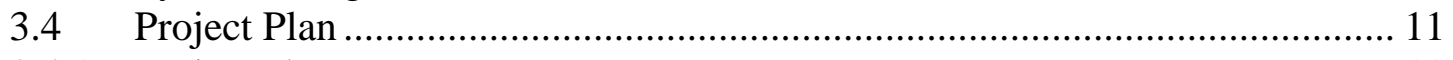

3.4.1 Project phases...................................................................................... 11

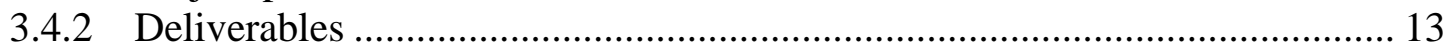

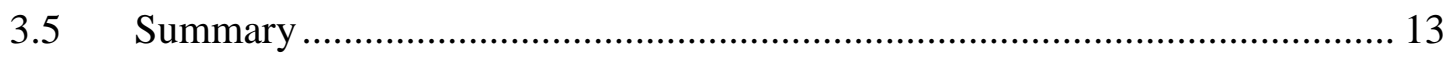

Chapter 4 - Database Design................................................................................... 15

4.1 Conceptual Data Model ................................................................... 15

4.2 Logical Data Model …………………………............................... 15

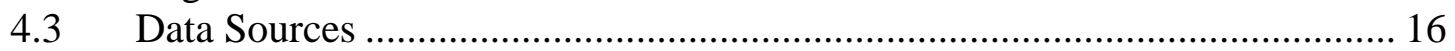

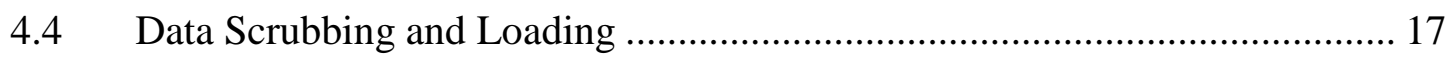

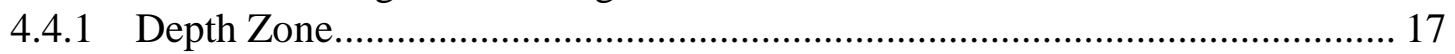

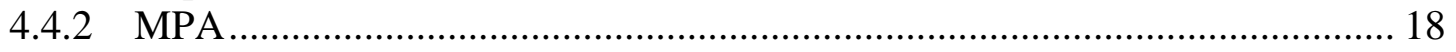

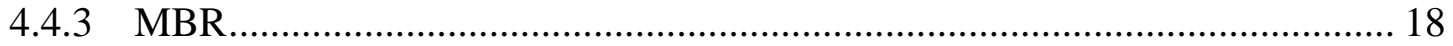

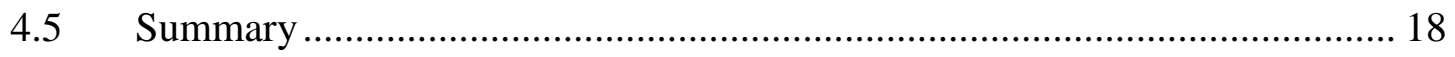

Chapter 5 - Implementation.................................................................................................. 21

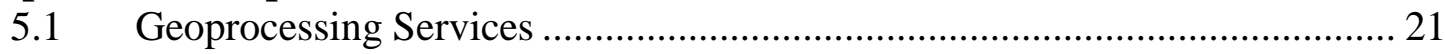

5.1.1 Protection Coverage of Marine Biogeographic Regions ................................. 21

5.1.2 Depth Distribution of an MPA................................................................ 23

5.2 Map Services and Feature Services ……………………………………... 23

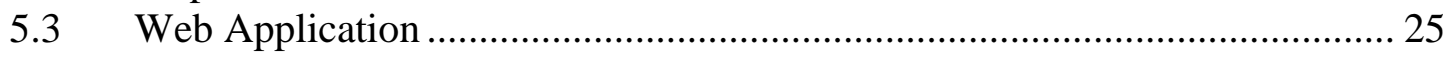

5.3.1 Interface Design ................................................................................. 25

5.3.2 Marine Protected Areas Information …………………………………….. 26 
5.3.3 Marine Biogeographic Regions Information ......................................... 26

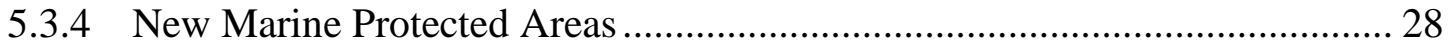

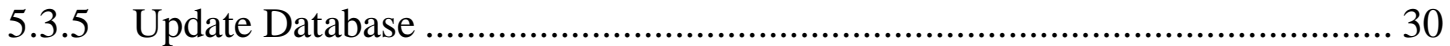

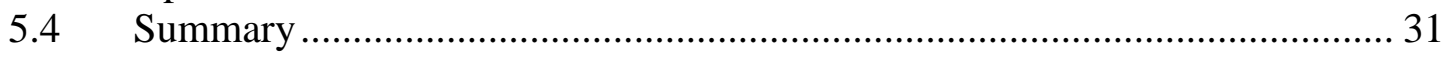

Chapter 6 - Use Cases ...................................................................................... 33

6.1 Use Case: Exploring Marine Protected Areas Information ........................... 33

6.2 Use Case: Exploring Marine Biogeographic Regions Information ................ 34

6.3 Use Case: Design and Update of Marine Protected Areas ............................. 35

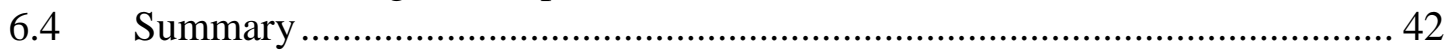

Chapter 7 - Conclusions and Future Work ............................................................... 43

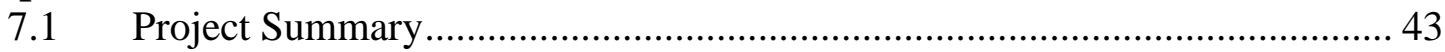

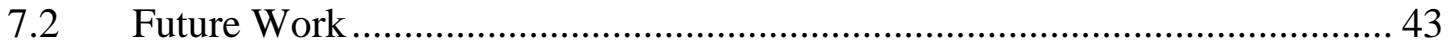

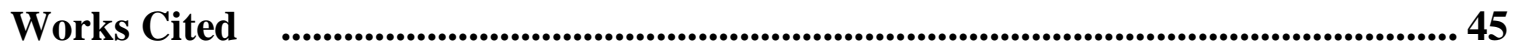

Appendix A. HTML Code

Appendix B. JavaScript Code.......................................................................................... 49

Appendix C. CSS Code.......................................................................................... 77 


\section{Table of Figures}

Figure 2-1: Marine Biogeographic Regions .................................................. 6

Figure 2-2: $\quad$ Protection Coverage of each Marine Biogeographic Region .............. 7

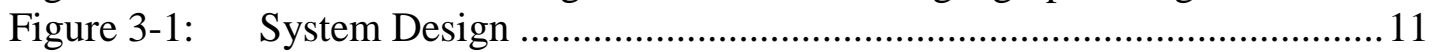

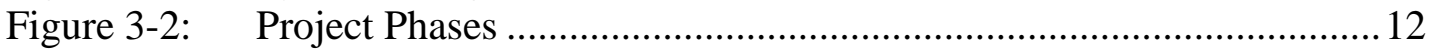

Figure 4-1: Conceptual Data Model ................................................................... 15

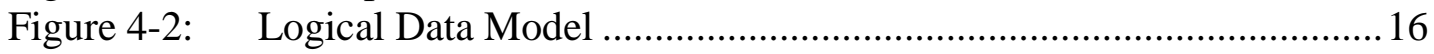

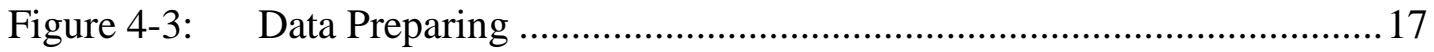

Figure 5-1: Program Flow Chart of the PCMBR Geoprocessing Tool .................2 21

Figure 5-2: $\quad$ Publishing the PCMBR Geoprocessing Service .................................22

Figure 5-3: Program Flow Chart of the DDMPA Geoprocessing Tool................23

Figure 5-4: $\quad$ Publishing the MPA Map and Feature Services ...............................24

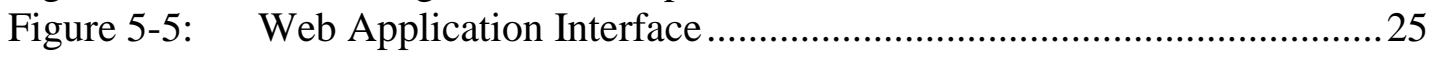

Figure 5-6: Program Flow Chart of the MPAs Information Panel ........................26

Figure 5-7: Interface of MBRs Information Panel..............................................2 27

Figure 5-8: $\quad$ Program Flow Chart of the MBRs Information Panel ....................... 27

Figure 5-9: Interface of the New MPAs Panel .....................................................28

Figure 5-10: Program Flow Chart of the New MPAs Panel....................................29

Figure 5-11: Interface of the Update Database Panel .......................................... 30

Figure 5-12: Program Flow Chart of the Update Database Panel ........................... 31

Figure 6-1: Marine Protected Areas Information Panel.......................................... 34

Figure 6-2: $\quad$ Marine Biogeographic Regions Information Panel ........................... 35

Figure 6-3: $\quad$ South Kuroshio Marine Biogeographic Region................................. 36

Figure 6-4: $\quad$ Eastern Philippines Marine Biogeographic Region ........................... 36

Figure 6-5: $\quad$ Draw New Marine Protected Areas ..................................................... 37

Figure 6-6: Calculate Protection Status of Marine Biogeographic Regions..........37

Figure 6-7: Calculate New Protection Status of Marine Biogeographic Regions 38

Figure 6-8: $\quad$ Save Marine Protected Areas .............................................................. 39

Figure 6-9: Define the Attributes of the Marine Protected Area in Design........... 39

Figure 6-10: $\quad$ Update the Marine Protected Area in Design ..................................... 40

Figure 6-11: Define the Attributes of a Marine Protected Area in Design ............. 41

Figure 6-12: $\quad$ Updated Marine Biogeographic Regions Information....................... 41 



\section{List of Tables}

Table 1. Functional Requirements ................................................................... 9

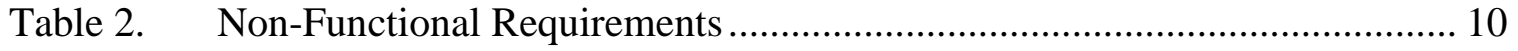

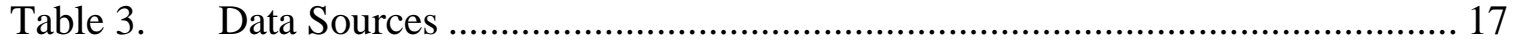

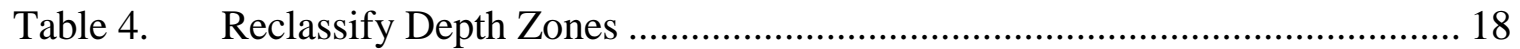





\section{List of Acronyms and Definitions}

$\begin{array}{ll}\text { API } & \text { Application Programming Interface } \\ \text { CSS } & \text { Cascading Style Sheets } \\ \text { DDMPA } & \text { Depth Distribution of an MPA } \\ \text { FTP } & \text { File Transfer Protocol } \\ \text { GIS } & \text { Geographic Information System } \\ \text { GSHHG } & \text { Global Self-consistent Hierarchical High-resolution Geography } \\ \text { HTML } & \text { Hypertext Markup Language } \\ \text { MBR } & \text { Marine Biogeographic Region } \\ \text { MCE } & \text { Multi-Criteria Evaluation } \\ \text { MCI } & \text { Marine Conservation Institute } \\ \text { MPA } & \text { Marine Protected Area } \\ \text { PCMBR } & \text { Protection Coverage of Marine Biogeographic Region } \\ \text { REST } & \text { Representational State Transfer } \\ \text { SRTM } & \text { Shuttle Radar Topography Mission } \\ \text { URL } & \text { Uniform Resource Locator } \\ \text { WGS } & \text { World Geodetic System }\end{array}$





\section{Chapter 1 - Introduction}

About $70 \%$ of the Earth's surface is covered by oceans. Marine and coastal ecological environment provide a variety of habitats for marine species. In turn, the richness of marine species strengthens the productivity and stability of marine ecosystems. However, losses in marine biodiversity are directly caused by many destructive activities, such as exploitation, pollution, and habitat destruction. Building marine protected areas (MPAs) is an essential way to safeguard marine biodiversity. However, only $2.07 \%$ of the world's oceans are protected in MPAs, and only $1.03 \%$ of the oceans is under strongly no-take marine reserve protection (Marine Conservation Institute, 2016). The other $1.04 \%$ of the oceans is under less restrictive reserves. In order to conserve marine biodiversity, scientists recommend that at least $20 \%$ of each marine biogeographic region (MBR) should be protected by MPAs in no-take marine reserves (Fernandes, et al., 2005). Besides increasing the percentage of oceans protected by MPAs, the balance of protection needs to be considered. The project would provide a web geographic information system (GIS) application for the Marine Conservation Institute to locate more MPAs to cover diverse MBRs. Also the application helps the institute collaborate with its partners when they propose new MPAs.

\subsection{Client}

The client for this project is the Marine Conservation Institute (MCI), a nonprofit ocean conservation organization. Working with scientists, politicians, government officials, and organizations, the institute is devoted to utilizing the latest marine science to protect the best places to stop marine biodiversity losses worldwide. The Global Ocean Refuge System proposed by the institute aims to protect $20 \%$ of each MBR of the oceans with MPAs before 2030 (Barragan-Paladines et al., 2015). The contact for this project was Dr. Lance Morgan, a marine biologist, MPA expert, and a conservation scientist. He is leading the development of the MPAtlas.org which can help people better understand the current state of global oceans' protection. The client helped define the scope and requirements analysis of this project, as well as providing data and feedback to the developer.

\subsection{Problem Statement}

The MCI needed a way to balance the protection coverage of the related MBRs and their depth zones when they propose new MPAs. They also needed a way to help them collaborate with their partners efficiently to propose new MPAs and update them to MPA database.

\subsection{Proposed Solution}

The proposed solution was a web GIS application developed with JavaScript, Cascade Style Sheet (CSS), and Hypertext Markup Language (HTML). The web application would let users inquire information of MPAs and MBRs. It would make users understand the present locations and coverage of MPAs, and current protection status of each MBR. 
Additionally, users can graphically design an MPA and examine the protection coverage of related MBRs and their depth zones with the application. It would help users balance the protection among MBRs to identify a location for the MPA when it is in design. After the MPA is approved, the application could update the MPA to the MPA database and update the protected area of related MBRs.

\subsubsection{Goals and Objectives}

The main goal of the project was to develop a solution for the client to propose new MPAs. Given these MPAs, the client attempted to balance the protection among MBRs and their depth zones. To obtain this goal, there were four objectives. The first objective was to develop a geoprocessing tool to calculate the area of each related MBR and its different depth zones protected in MPAs. The second objective was to develop a geoprocessing tool to calculate the area of depth zones across a new MPA. The third objective was to publish these two tools as two geoprocessing services. The last objective was to develop a web application which lets users graphically design a new MPA and update it to an existing MPA database. The geoprocessing services were used in the web application.

\subsubsection{Scope}

The study area of this project was global marine areas. The main deliverable of the project is the web application, which helps users graphically propose a new MPA, execute the geoprocessing services to help users balance the protection among MBRs by proposing the new MPA, and update the new MPA to existing MPA database. All the services which were consumed in the web application were published using ArcGIS for Server.

The project was limited to considering the percentage of each MBR protected in MPAs, and some other factors that need to be considered when people propose a new MPA. The water depth data were reclassified to three classes based on the thresholds defined by the client which were 0-200 m, 200-1,000 m, and greater than 1,000 m. The web application will be guaranteed to work in Google Chrome web browser. It might work in other browsers, but it is not guaranteed.

\subsubsection{Methods}

Global data were used in this project. The geographic coordinate system of the data was World Geodetic System Datum 1984 (WGS84). The data consisted of MPA data provided by the client, MBR data from Marine Ecoregions of the World (MEOW) shapefiles, bathymetry data from Shuttle Radar Topography Mission 30 Plus (SRTM30 PLUS) data, and coastline data from Global Self-consistent Hierarchical High-resolution Geography (GSHHG) database.

The geoprocessing tools were developed as Python scripts. The feature services, map services, and geoprocessing services were published using ArcGIS for Server. All the data that were used by these services were hosted by an enterprise geodatabase registered with ArcGIS for Server. The web application was developed using JavaScript, HTML, and CSS. The map services and the feature services were used to display and edit data in 
the web application. A new MPA which is defined by a user is an input to call the geoprocessing services.

\subsection{Audience}

The main audiences of the report were the MCI staff and other nonprofit ocean conservation organizations currently engaged in designing MPAs, as well as other GIS professionals and developers who are considering developing web applications for marine conservation.

\subsection{Overview of the Rest of this Report}

Chapter 2 gives the background of MPAs and MBRs, reviews the previous GIS applications for marine protection, and introduces the web GIS technology. Chapter 3 describes the problem statement, the requirements analysis, the system design, and the project plan. Chapter 4 provides the details of the database design. Chapter 5 presents the implementation process of the project, Chapter 6 includes the results and use cases of the web application, and Chapter 7 summarizes the project and describes possible future work. 



\section{Chapter 2 - Background and Literature Review}

Geographic information system (GIS) technology is applied widely in evaluating marine protected areas (MPAs) and managing marine resources. This chapter provides background and key concepts related to the project. Section 2.1 introduces marine biogeographic regions (MBRs) and biogeographic classification methods. Section 2.2 describes the current distribution of MPAs and requirements for proposing new ones. Section 2.3 discusses how previous applications in marine conservation used GIS technology to help design MPAs and manage fisheries resources. Section 2.4 introduces the web GIS technology and its advantages.

\subsection{Marine Biogeographic Regions}

In 1805, the first biogeographic map was published by Lamarck and Candolle in the third edition of Flore Française (Ebach \& Goujet, 2006). Biogeographic classifications provided an essential foundation for evaluating representativeness to protect worldwide biodiversity (Olson \& Dinerstein, 2002).

Researchers used various criteria to distinguish marine biogeographic characteristics. The researchers developed several global systems to improve the understanding of marine biogeography and management of marine resources. Briggs $(1974,1995)$ defined a system of coastal and shelf provinces based on their endemism. Longhurst (1998) designed a two-tier pelagic system of biomes and biogeochemical provinces based on chlorophyll data. However it was not suitable to apply in complex systems of coastal water. Large marine ecosystems were proposed by several regional experts (Sherman \& Alexander, 1989; Sherman et al., 2005). It focused on fisheries resources, pollution, productivity, and oceanographic processes.

In order to analyze and protect marine biodiversity, Spalding et al. (2007) presented a biogeographic multiscale classification for the global coastal and shelf areas. They proposed a global nested biogeographic system: the Marine Ecoregions of the World (MEOW) which included 12 realms, 62 provinces, and 232 ecoregions - namely, biogeographic regions. Realms are the largest units. Each of them contains multiple provinces. Every province includes several regions. MBRs are spatial units hosting distinct and homogeneous species. The dominate biogeographic forcing factors defined the location of MBRs. Spalding suggested the most appropriate outer boundary for these three units is the 200-meter isobaths curve. Because it is widely used for corresponding to the shelf edge that has plenty of overlaps between shelfs and slopes. Additionally, the data from deeper water would hide or change the biogeographic patterns represented by MEOW system. Figure 2-1 shows the map of MBRs, and the numbers on the map indexing each MBR individually. 


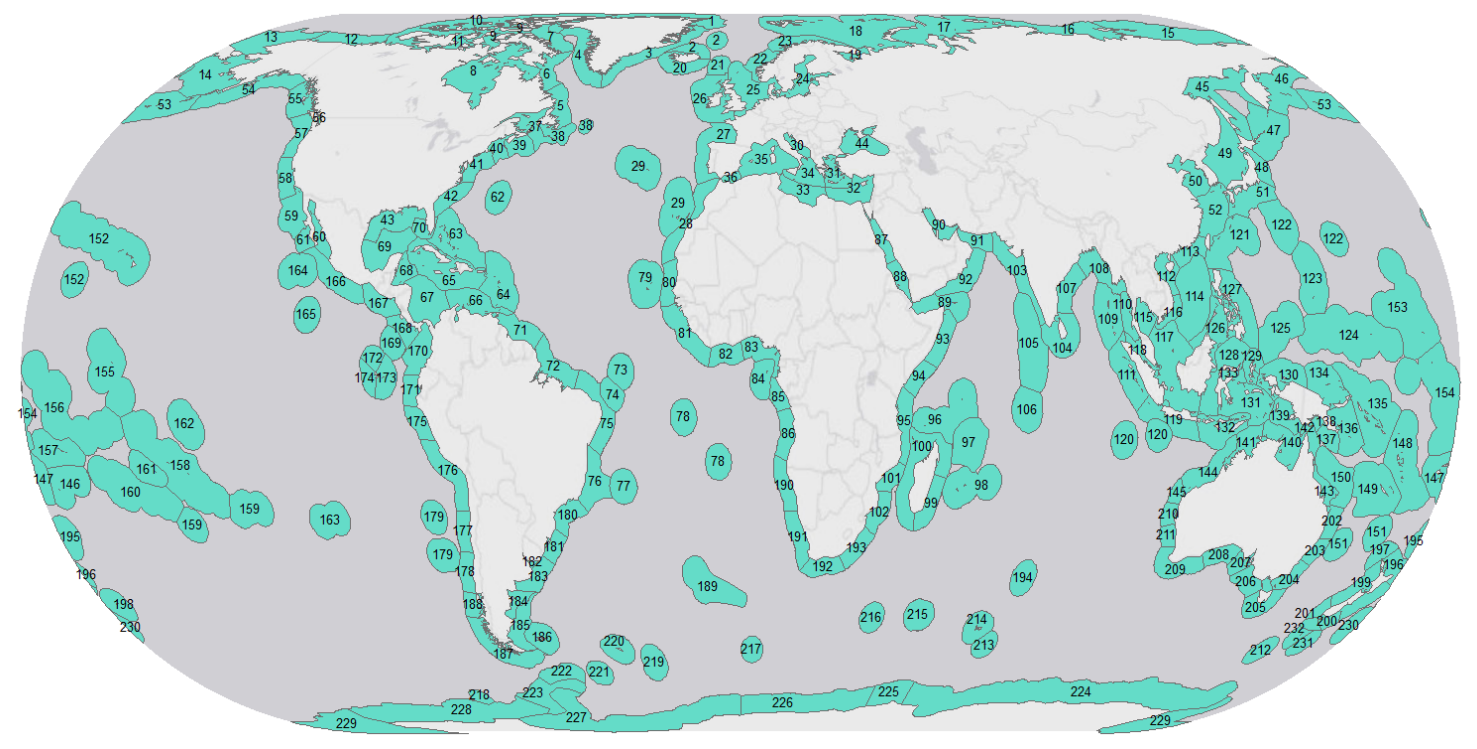

Figure 2-1: Marine Biogeographic Regions

\subsection{Marine Protected Areas}

Establishing MPAs is recognized as an effective strategy to preserve marine biodiversity (Halpern \& Warner, 2002; Roberts et al., 2003). However, only $2.07 \%$ of the world's oceans are designated as MPAs, and only $1.03 \%$ of the oceans is under strong no-take marine reserve protection (Marine Conservation Institute, 2016). Another $1.04 \%$ of the oceans in MPAs is under less restriction. No-take indicates that no extractive activities can be conducted in protected areas (Marine Conservation Institute, 2015). It provides the strongest protection from the harmful effects of activities, such as fishing, mining, and drilling for oil and gas.

The challenges of establishing MPAs include slow progress, legislative hurdles, and the constraints of human needs. Therefore, MPAs are often limited to regions that are unappealing for human use. Besides proposing more MPAs to increase protection coverage, scientists are also concerned with maintaining or increasing the equality of protection. In order to protect marine biodiversity, scientists recommend that a minimum of $20 \%$ of each MBR be protected at the no-take level (Fernandes et al., 2005). Barr et al. (2011) applied the Gini coefficient to measure the equality in the protection of MPAs. The Gini coefficient was initially adapted to quantify the inequality of income among countries (Gini, 1921). The Global Ocean Refuge System proposed by Marine Conservation Institute established a target to protect $20 \%$ of the ecosystem in each MBR of the global oceans with MPAs by 2030 (Barragan-Paladines et al., 2015). Figure 2-2 illustrates the current protection status of each MBR. The protection of many MBRs does not achieve the target of the institute. Although several MBRs have more than $20 \%$ protected, they are not all protected under the no-take level to fulfill the researchers' 
recommendation. More efforts and effective ways would be required to propose new MPAs wisely.

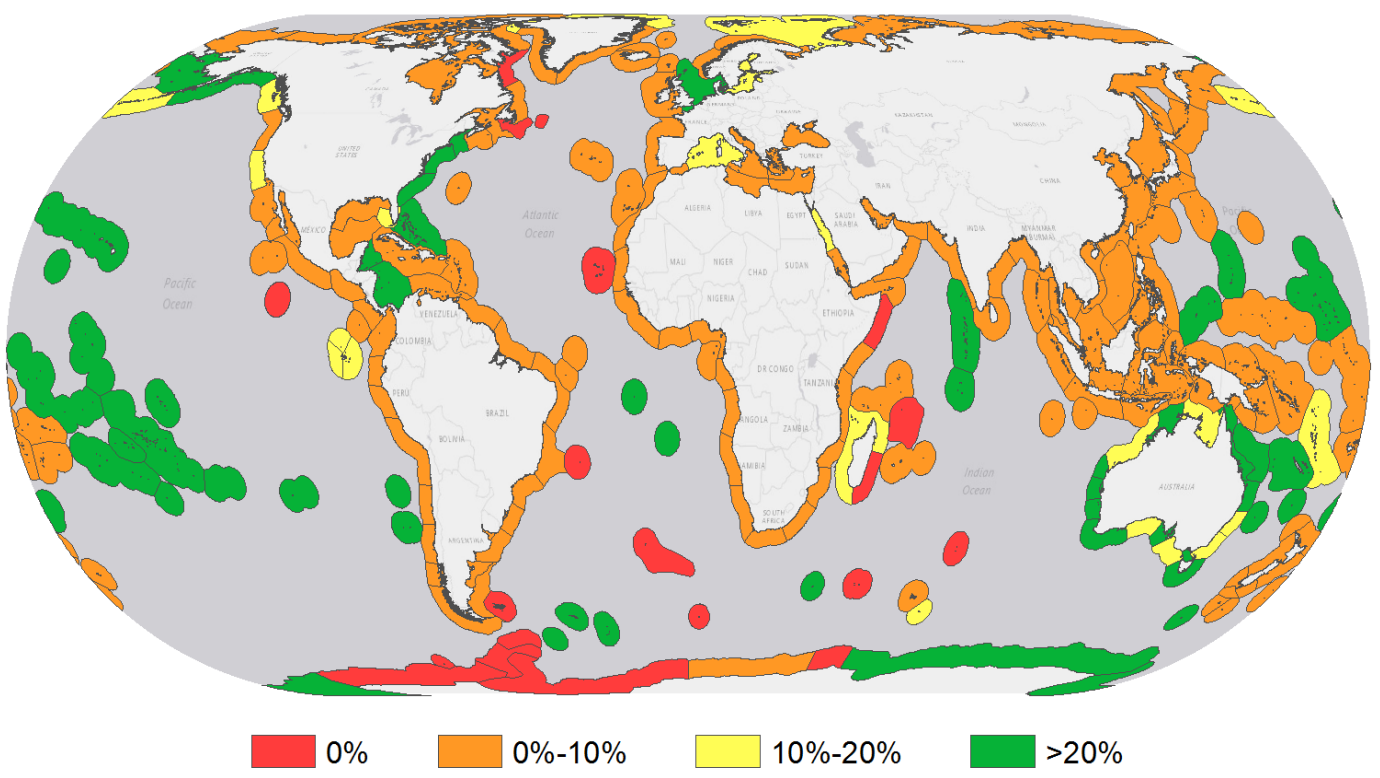

Figure 2-2: Protection Coverage of each Marine Biogeographic Region

\subsection{GIS Application for Marine Protection}

GIS technology has been applied widely in marine environmental conservation, varying from the preservation of the diversity of species and their habitats to the management of natural resources.

GIS could combine multisource spatial data to provide visualization capabilities to help users better understand how MPAs might be developed. A GIS database could be applied to integrate indigenous knowledge, socioecological behavior, artisanal fishing data, biophysical processes, and other information to assist in the design of MPAs (Aswani \& Lauer, 2006). Harris and Whiteway (2009) analyzed seafloor geomorphology and seascape classification maps to identify the candidates for high sea MPAs. MultiCriteria Evaluation (MCE) was a well-established optimization method used in allocation and decision support of land-use resources. Based on GIS, MCE and fuzzy sets were used to objectively identify locations for future MPAs (Wood \& Dragicevic, 2007).

GIS could be not only applied in MPA design but also used in the management of marine fisheries resources and environments. For example, in order to plan, monitor, and manage mangroves of the Kiunga MPA, digital vegetation maps produced on a GIS platform were used to store, retrieve, and analyze spatial information efficiently (Kairo, Kivyatu, \& Koedam, 2002). A biogeographic process using GIS and sampling methods evaluated existing MPAs, which guided better and more effective management decisions (Friedlander, Brown, \& Monaco, 2007). With GIS, the distribution of French grunt habitats and species habitat affinities showed and predicted utilization patterns of species habitat for the fish (Kendall, Christensen, \& Hillis-Starr, 2003). Fine-resolution mapping of land-cover types implemented in aerial photography, GIS, and ground data could be 
used in an estuarine system for conservation and management of estuarine habitats and their species (Zharikov, Skilleter, Loneragan, Taranto, \& Cameron, 2005). To achieve the management of marine ecosystems, the GIS approach could be applied to improve researchers' understanding of ecosystems' patterns and processes. Marine conservation initiatives faced the challenge of fulfilling two conflicting objectives: biodiversity conservation and fisheries' profit maximization. GIS and other spatial technologies could be applied to build digital oceans and realize the conversion from data, information, and knowledge into decision-making despite uncertainty and complexity.

\subsection{Web GIS Application}

Web GIS is a distributed information system that uses internet technology to enable communication between server and client (Fu \& Sun, 2010). Its basic components are the geodatabase, GIS server, web server, and the clients. The web GIS system supports the internet protocol suite (TCP/IP), the File Transfer Protocol (FTP), and the Hypertext Transfer Protocol (HTTP), which means that the web GIS can connect to data anywhere. Users can use a web GIS application to find a variety of spatial data and the related text data from the internet. Also, users can process a variety of spatial analyses with web GIS. Compared with traditional GIS, web GIS technology has several advantages (Fu \& Sun, 2010), including the following:

Global reach - Users can access the latest data from multiple servers in different places;

Better cross-platform capabilities - No matter what kind of machine the server/client is and no matter what kind of GIS software the server uses, a web browser can be used to access spatial data;

Low cost per web GIS user - Traditional GIS technologies need expensive professional GIS software on each client site, but users usually only need basic functions. Web GIS users just need a web browser;

Ease of use and maintenance — Using a common web browser reduces the operational complexity of GIS, which makes it easier for users to apply GIS technology.

\subsection{Summary}

This chapter illustrated the concept of MBRs and MPAs. Not only more MPAs but also equal protections are needed to stop the loss of marine biodiversity. This chapter also reviewed some GIS approaches for marine conservation that integrated data from multiple sources and provided visualization to develop MPAs and manage marine resources. Lastly, this chapter introduced web GIS technology and its advantages compared to traditional GIS. 


\section{Chapter 3 - Systems Analysis and Design}

This chapter reviews the client's problem in Section 3.1 and identifies both functional and non-functional requirements provided by the client in Section 3.2. Section 3.3 describes the system design to show the major components of the project, and Section 3.4 discusses the project plan to explain the major tasks of the project.

\subsection{Problem Statement}

The problem addressed in the project was to analyze at a global scale where new marine protected areas (MPAs) were needed in different marine biogeographic regions (MBRs). Given a new MPA, the Marine Conservation Institute needed a way to balance the protection coverage of related MBRs and their depth zones. Also, the client needed a way to help it collaborate with its partners efficiently during the process of proposing new MPAs and update these MPAs to MPA database.

\subsection{Requirements Analysis}

The requirements analysis was critical for defining solutions to meet the client's needs. A detailed requirements analysis can avoid scope creep, reworking, and issues of quality. Requirements contained functional requirements and non-functional requirements. They were set based on discussion with the client.

\subsubsection{Functional requirements}

Functional requirements are the capabilities which must be provided by the web GIS application. The details of functional requirements related to the project are listed in Table 1.

\section{Table 1. Functional Requirements}

\begin{tabular}{|c|l|}
\hline Requirement & Description \\
\hline $\begin{array}{c}\text { Inquire about } \\
\text { MPAs }\end{array}$ & $\begin{array}{l}\text { A user should be able to inquire information about each MPA, } \\
\text { including the name, percentage of no-take areas, and depth } \\
\text { distribution. }\end{array}$ \\
\hline $\begin{array}{c}\text { Inquire about } \\
\text { MBRs }\end{array}$ & $\begin{array}{l}\text { A user should be able to get information about each MBR, } \\
\text { including the name, the realm, and the province that the MBR } \\
\text { belongs to, the protection percentage, depth distribution, and the } \\
\text { protection percentage of different depth zones. }\end{array}$ \\
\hline $\begin{array}{c}\text { Draw an MPA in } \\
\text { design }\end{array}$ & $\begin{array}{l}\text { A user should be able to graphically define an MPA in design and } \\
\text { get its area and perimeter automatically. }\end{array}$ \\
\hline
\end{tabular}




\begin{tabular}{|c|l|}
\hline Requirement & Description \\
\hline $\begin{array}{c}\text { Calculate } \\
\text { protection } \\
\text { coverage of MBRs }\end{array}$ & $\begin{array}{l}\text { A user should be able to calculate the protection coverage of the } \\
\text { MBRs related to a new MPA and the protection coverage of their } \\
\text { depth zones. }\end{array}$ \\
\hline $\begin{array}{c}\text { Save attributes of a } \\
\text { designed MPA }\end{array}$ & $\begin{array}{l}\text { A user should be able to change and update the attributes of a } \\
\text { designed MPA. }\end{array}$ \\
\hline $\begin{array}{c}\text { Update MPA } \\
\text { database }\end{array}$ & $\begin{array}{l}\text { A user should be able to update a designed MPA to the MPA } \\
\text { database }\end{array}$ \\
\hline $\begin{array}{c}\text { Update MBR } \\
\text { database }\end{array}$ & A user should be able to update protection coverage of the related \\
MBRs when a new MPA is updated.
\end{tabular}

\subsubsection{Non-functional requirements}

Non-functional requirements focus on the performance of deliverables, as well as technical and operational requirements that support the functional requirements. They are associated with usability, accessibility, and operational environment. The details of nonfunctional requirements related to the project are listed in Table 2.

\section{Table 2. Non-Functional Requirements}

\begin{tabular}{|l|l|}
\hline Requirement & Description \\
\hline Operating System & $\begin{array}{l}\text { Windows 7, 64-bit operating system, 8 GB RAM, and } \\
\text { 3.00 GHz processor speed }\end{array}$ \\
\hline Esri ArcMap 10.3 & $\begin{array}{l}\text { The platform was used to work with the geodatabase } \\
\text { and the geoprocessing tools, as well as to publish the } \\
\text { feature services, the map services, and the } \\
\text { geoprocessing services }\end{array}$ \\
\hline Enterprise Geodatabase & $\begin{array}{l}\text { The enterprise geodatabase is required to hold the } \\
\text { feature classes which are published as the feature } \\
\text { services with ArcGIS for Server. }\end{array}$ \\
\hline $\begin{array}{l}\text { Esri ArcGIS for Server } \\
10.4\end{array}$ & $\begin{array}{l}\text { The server needs to host the map services, the feature } \\
\text { services, and the geoprocessing services }\end{array}$ \\
\hline $\begin{array}{l}\text { Google Chrome Web } \\
\text { Browser }\end{array}$ & The browser is used to test the web application \\
\hline
\end{tabular}




\subsection{System Design}

The system consisted of three components as illustrated in Figure 3-1.

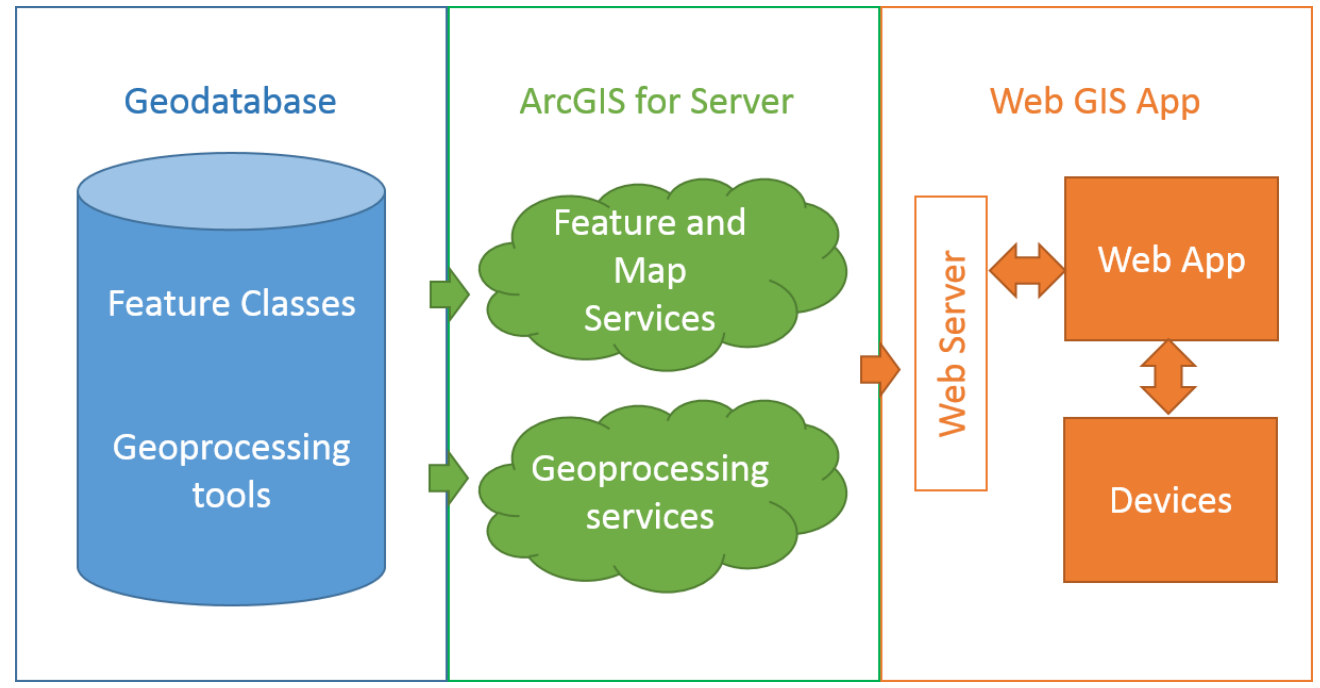

Figure 3-1: System Design

The geodatabase contained the feature classes and the geoprocessing tools. The feature classes had all the attributes which were required in the web application. There were two geoprocessing tools. Given an MPA, the first geoprocessing tool was used to select the related MBRs and to update the protected area's status. The second geoprocessing tool was used to find the depth distribution of the MPA.

The feature classes were published as map services and feature services. The geoprocessing tools were published as geoprocessing services. All the services were published by ArcGIS for Server and are utilized by the web application.

The web application interacts with a user, and communicates with the server to perform operations requested by the user.

\subsection{Project Plan}

The project plan helped to keep the process of the project on track. It included project phases, deliverables, and assumptions. This section gives further details of these three components.

\subsubsection{Project phases}

There were four phases in the project: design, implementation, acceptance, and deployment. The details of each phase are given in Figure 3-2. 


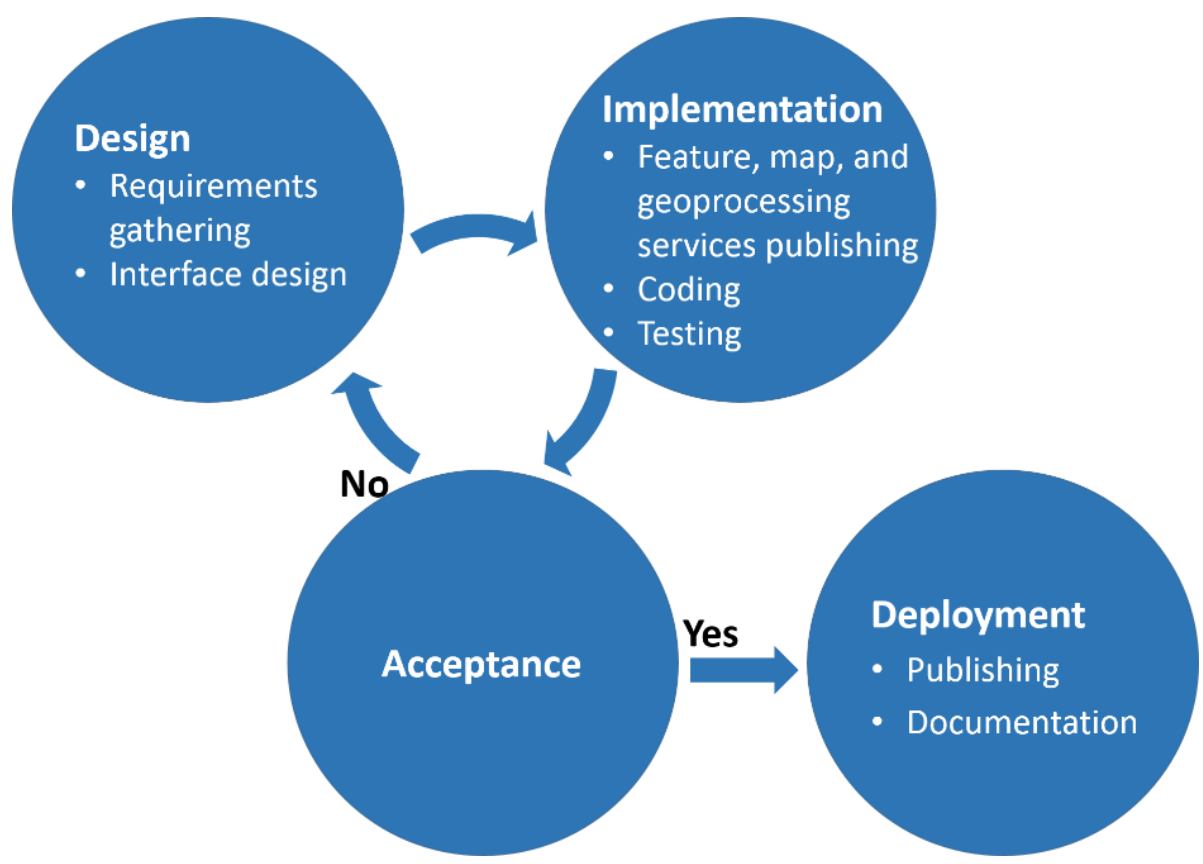

\section{Figure 3-2: Project Phases}

The web application was developed by using an agile system development life cycle. The requirements were changed during the development process of the project, so the design, implementation, and acceptance phases were in a cycle when new requirements were added to the web application. When the web application was accepted by the client, it was released in the deployment phase.

In the design phase, the first task was to gather requirements of the web application from the client. The second task was to design the interface of the web application. The interface was modified based on the changes of requirements.

In the implementation phase, the first task was to publish the feature services, the map services, and the geoprocessing services. The map services are used to display the data and the feature services are used to edit the data through the web application. Regarding a new MPA, one of the geoprocessing services can be applied to calculate the new protection coverage in the related MBRs and the new protection coverage in different depth zones within these MBRs. Another geoprocessing service can be applied to calculate depth distribution of this new MPA. The second task was to develop the web application using JavaScript, Hypertext Markup Language (HTML), and Cascading Style Sheets (CSS). The third task was to test the web application to fix the bugs. It included both unit testing and integration testing. Unit testing was used for testing each function of the web application. Integration testing was used for testing the web application when these functions were combined.

In the deployment phase, the web application was released. The user manual and related documents were provided to the client. 


\subsubsection{Deliverables}

The main deliverable of the project was the web application as a package which contained .html, .css, and .js files. Other deliverables were the feature services, the map services, and the geoprocessing services which were published with ArcGIS for Server.

\subsection{Summary}

This chapter revisited the problem addressed in the project, and identified the functional and non-functional requirements. It also discussed the system design and project plan. They were very significant to the success of the project. 



\section{Chapter 4 - Database Design}

Chapter 4 discusses the conceptual and logical data model, the different data sources, as well as the preprocessing of the required data in the project. Section 4.1 describes the entities needed in the project and relationships among them. Section 4.2 outlines the structure of the geodatabase which included the feature classes used in the web application. Section 4.3 states the data sources, followed by Section 4.4 that describes the process of data preparing.

\subsection{Conceptual Data Model}

A conceptual data model includes all the entities and their relationships required to address the problem of the project. They are earth surface, oceans, lands, marine protected areas (MPAs), and marine biogeographic regions (MBRs). Figure 4-1 illustrates the conceptual model for this project.

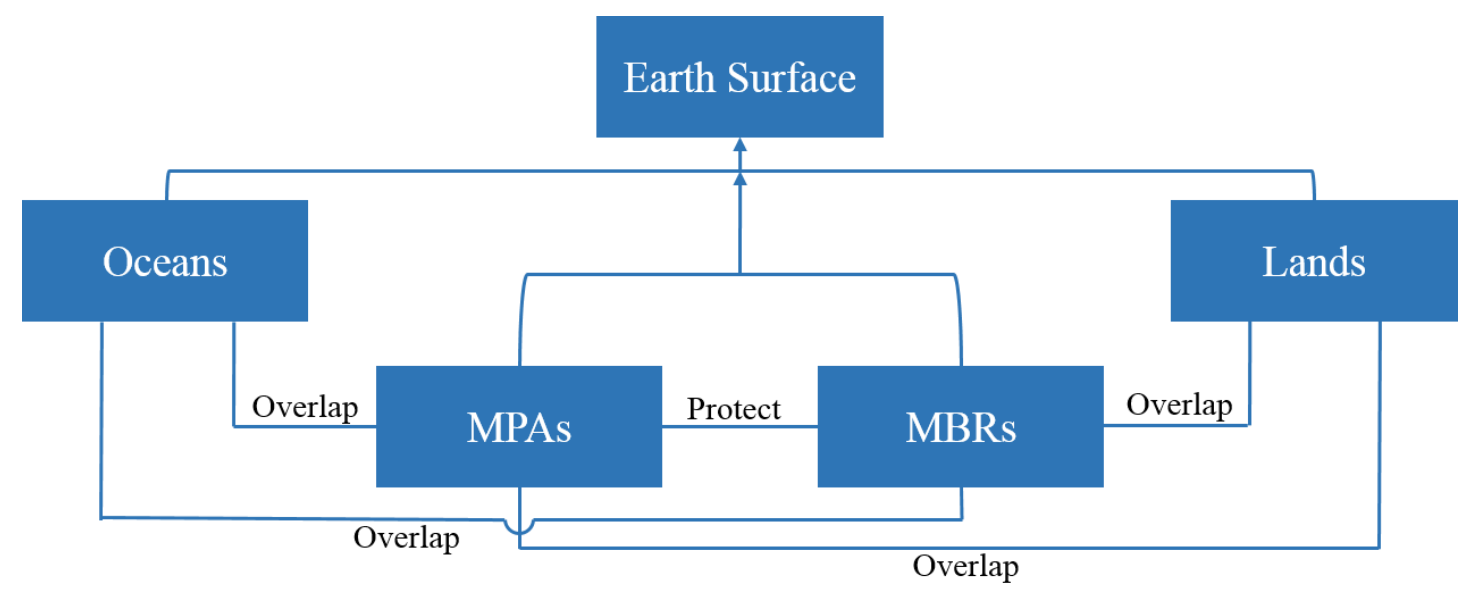

\section{Figure 4-1: Conceptual Data Model}

The earth's surface can be broken into ocean and land segments. Some pieces of the earth surface are occupied by MPAs and MBRs. They overlap with each other, which means MBRs are protected by MPAs. Both of them have overlapping regions with oceans and lands. The land areas which are covered by marine protected areas has freshwater resources. MBRs cover coastal and shelf areas.

\subsection{Logical Data Model}

A logical data model gives details about the data requirements to solve the problems that the project addressed in the conceptual data model. Because of the limitations of technology, earth surface, oceans, and lands are difficult to present in a geodatabase. And the project is focused on MPAs, MBRs, and the relationship between them. In a water area, MPAs and MBRs not only have water surface but also have different water depths. So instead of earth surface, oceans, and lands, the logical data had depth information as 
attributes of MPAs and MBRs. Figure 4-2 explains the logical data model which represents the structure of the geodatabase.

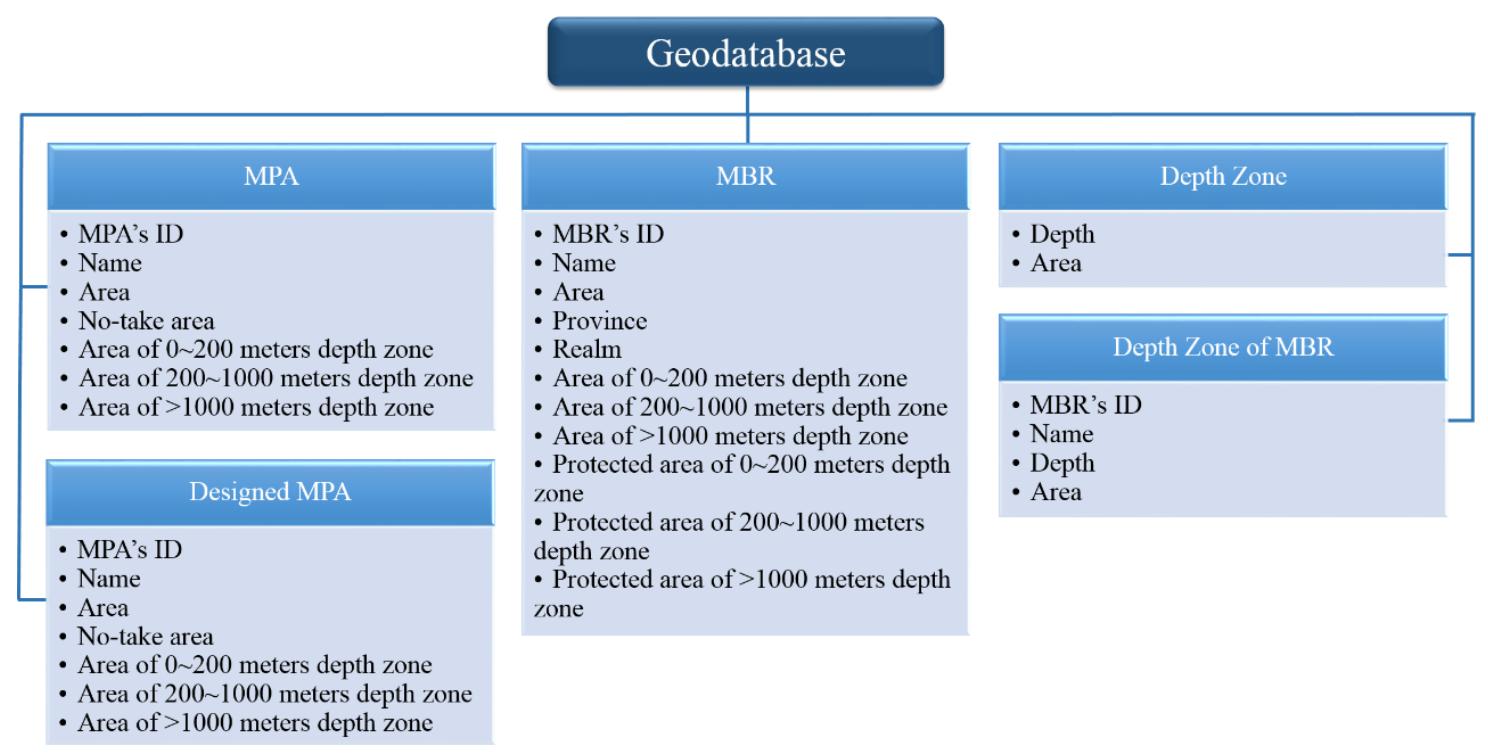

\section{Figure 4-2: Logical Data Model}

The geodatabase had five polygon feature classes. The first feature class was the MPA which held information of existing MPAs. The second feature class was the Designed MPA which had the same schema as the MPA feature class. It held new MPAs that were being designed by users. After these MPAs are approved, they can be updated to the MPA feature class. The third feature class was the MBR which hold information about MBRs. Each record represents an MBR. The fourth feature class was the Depth Zone which held three different depth zones (0-200 meters, 200-1,000 meters, and >1,000 meters) of global area. The last feature class was the Depth Zone of MBR. Each record represented a depth zone within an MBR. This was a derived feature class from the third and fourth feature classes. The reason for keeping it in the geodatabase was that it was an input data of the geoprocessing service. The third and fourth feature classes were global data. The last feature class was used instead of the global water depth zones because considerable time would be saved when the geoprocessing service was executed. The first three feature classes acted as interactive layers in the web application; these three feature classes were the input data of geoprocessing services.

\subsection{Data Sources}

The client (Marine Conservation Institute) provided the MPA geodatabase and its metadata. Other required data were downloaded from externally trustworthy sources' websites and they all included metadata or the link of metadata. Table 3 lists name, source, and format of the data. 
Table 3. Data Sources

\begin{tabular}{|l|l|l|}
\hline \multicolumn{1}{|c|}{ Data } & \multicolumn{1}{|c|}{ Source } & \multicolumn{1}{c|}{ Format } \\
\hline $\begin{array}{l}\text { MPA } \\
\text { (MEOW) }\end{array}$ & Marine Conservation Institute & Geodatabase \\
\hline $\begin{array}{l}\text { Shuttle Radar Topography Mission } \\
\text { 30 Plus (SRTM30 PLUS) }\end{array}$ & $\begin{array}{l}\text { Sorld Wildlife Fund } \\
\text { Shollite Geodesy Research }\end{array}$ & Raster \\
\hline $\begin{array}{l}\text { Global Self-consistent Hierarchical } \\
\text { High-resolution Geography } \\
\text { (GSHHG) }\end{array}$ & $\begin{array}{l}\text { National Centers for } \\
\text { Environmental Information, } \\
\text { NOAA }\end{array}$ & Shapefile \\
\hline
\end{tabular}

\subsection{Data Scrubbing and Loading}

The original MPA and MBR data did not have depth information and the MBR data did not have the protection area of each MBR. Raster data needed to be converted to the feature class format. Before loading these data into the geodatabase mentioned in the logical data model, they required the preprocessing to make sure they had required attributes. The geographic coordinate system of these data was World Geodetic System Datum 1984 (WGS84) and they did not have projection coordinate system, so the areas of depth zones and protected areas were calculated as surface area using a Python script (Esri, 2016). Figure 4-3 illustrates the preparation processing of the data before loaded in the geodatabase of this project. They are represented by dark blue rectangles in Figure 4-3.

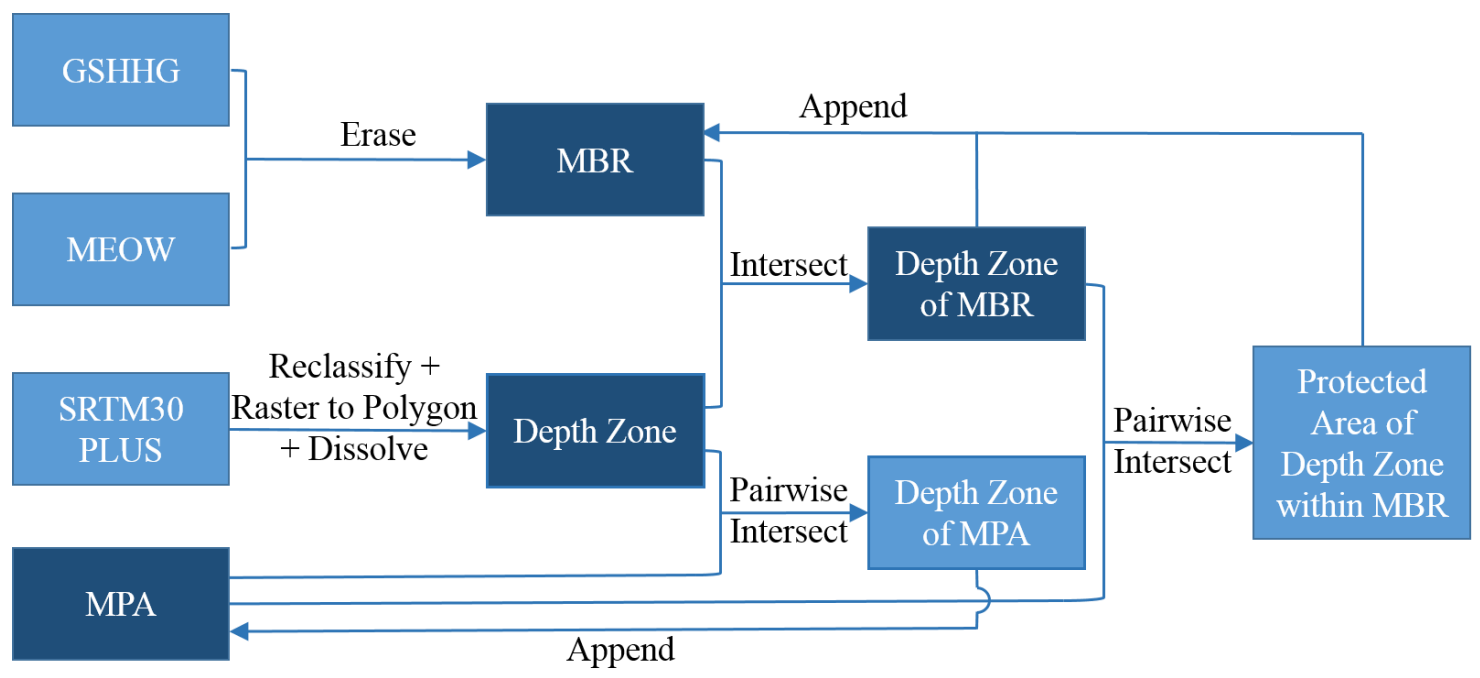

Figure 4-3: Data Preparing

\subsubsection{Depth Zone}

Shuttle Radar Topography Mission 30 Plus (SRTM30 PLUS) were raster data which combined SRTM land topography with measured and estimated seafloor topography. The 
client provided the depth thresholds which were 0-200 m, 200-1,000 m, and greater than $1,000 \mathrm{~m}$ to classify the water depth data into three classes. Table 4 shows the ranges of pixel values in the input raster data and the pixel values in the output raster data. After classification, the output raster was converted to a polygon feature class. Then the feature class was dissolved based on the same class values. In the end, this feature class had three classes and each record was a depth zone.

\section{Table 4. Reclassify Depth Zones}

\begin{tabular}{|c|c|}
\hline Input Range & Output Value \\
\hline Value $>=-200$ & 0 \\
\hline$-1,000<$ Value $<=-200$ & -200 \\
\hline Value $<=-1,000$ & $-1,000$ \\
\hline
\end{tabular}

\subsubsection{MPA}

The original MPA feature class needed to be intersected with the Depth Zone feature class to obtain the depth zone coverage within each MPA. Since there were some overlapping MPAs, the geoprocessing method was the Pairwise Intersect Tool (ArcGIS TeamPython, 2013) instead of the Intersect Tool. The output of the Intersect contained some features which came from the intersection of the overlapping MPAs. However, these features were not needed for the project. The Pairwise Intersect Tool just compared each feature in the MPA feature class with each feature in the Depth Zone feature class to provide the required results. The area field of three depth zones in the Depth Zone of MPA feature class was appended to the MPA feature class as three fields using a Python script developed in the project.

\subsubsection{MBR}

The original Marine Ecoregions of the World (MEOW) data covered both marine areas and land areas. Since the project focused on the oceans, the MEOW data were clipped using Global Self-consistent Hierarchical High-resolution Geography (GSHHS) coastline data to get the MBR feature class which only covered the marine areas. This feature class did not have information about covered depth zones or area of protected depth zones. The area of cover depth zones could be obtained from the Depth Zone of MBR feature class which was the intersection of the MBR and the Depth Zone feature classes. The area of protected depth zones could be attained from the MPA and the Depth Zone of MBR feature class with the Pairwise Intersect Tool. Using the same Python script mentioned in 4.4.2, three fields were added to the attributes of the MBR feature class representing how much of these three water depth zones is covered. Another three fields were also added to represent how much of each water depth zone is protected by MPAs.

\subsection{Summary}

This chapter discussed the conceptual data model, the logical data model, data sources, and the process of preparing the data. The conceptual and logical data models described the entities and their relationships, as well as the structure of the geodatabase. All the data 
came from reliable sources with the metadata. In order to have the required attributes, the data which were used in the web application needed to be preprocessed before they were loaded in the geodatabase. 



\section{Chapter 5 - Implementation}

Chapter 5 discusses the implementation of the web services and the web application. Section 5.1 discusses the development of two Python Script tools and how they were published as the geoprocessing services. Section 5.2 describes the map services and feature services used in the application, and how to publish them using ArcGIS for Server. Section 5.3 illustrates the interface design and how the web application was developed.

\subsection{Geoprocessing Services}

There were two geoprocessing services consumed in the web application. They were developed using Python and the ArcGIS 10.3 Toolbox, and published with ArcGIS for Server.

\subsubsection{Protection Coverage of Marine Biogeographic Regions}

The protection coverage of marine biogeographic regions (PCMBR) geoprocessing service's output reflects the influence of a marine protected area (MPA) to its related marine biogeographic regions (MBRs). The geoprocessing services contained a geoprocessing task which was a geoprocessing tool running with ArcGIS for Server. Its outputs were also managed by the server. The geoprocessing tool was a Python Script which was added to an ArcGIS 10.3 Toolbox. It had four input parameters which comprised the Designed MPA, Depth Zone of MBR, MPA, and MBR feature classes. Figure 5-1 shows the program flow chart of the PCMBR geoprocessing tool.

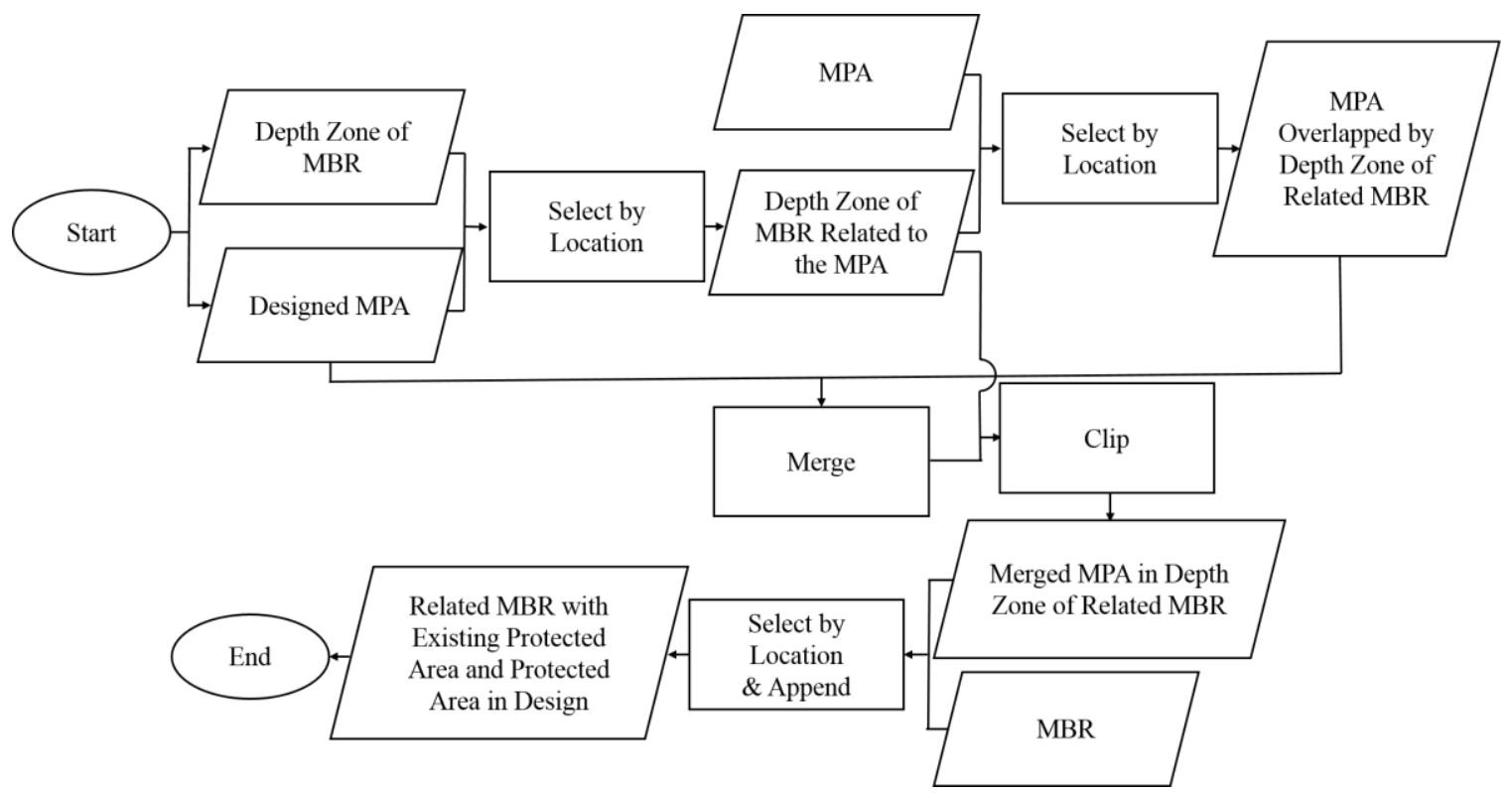

Figure 5-1: Program Flow Chart of the PCMBR Geoprocessing Tool 
Given an MPA in polygon format, the PCMBR geoprocessing tool could select the MBRs which overlapped with this MPA using the Select by Location method in ArcMap 10.3. The geoprocessing tool could then calculate how much of those MBRs and their depth zones are protected by existing MPAs and this new MPA using the Clip tool from ArcMap10.3. The output of the PCMBR geoprocessing service contained the MBRs which related to this MPA. Each MBR had the area, the area of three depth zones, the existing protected area, and the protected area of three depth zones. The area unit was a square kilometer.

Before publishing the PCMBR geoprocessing service, the PCMBR geoprocessing tool must be executed successfully in ArcMap10.3. Then its result can be shared as the geoprocessing service and a corresponding geoprocessing task is created. Figure 5-2 shows the settings for publishing this geoprocessing service. The service has geoprocessing capability and it uses an asynchronous execution mode. For the four input parameters used in the geoprocessing tool, the input mode of the Designed MPA feature class (see InputNewMPA in Figure 5-2) was set as "User defined value," which means that a user needs to provide the parameter value. The other three inputs were set as "Constant value," which means the user cannot change them. They were not the task parameters when the service was published.

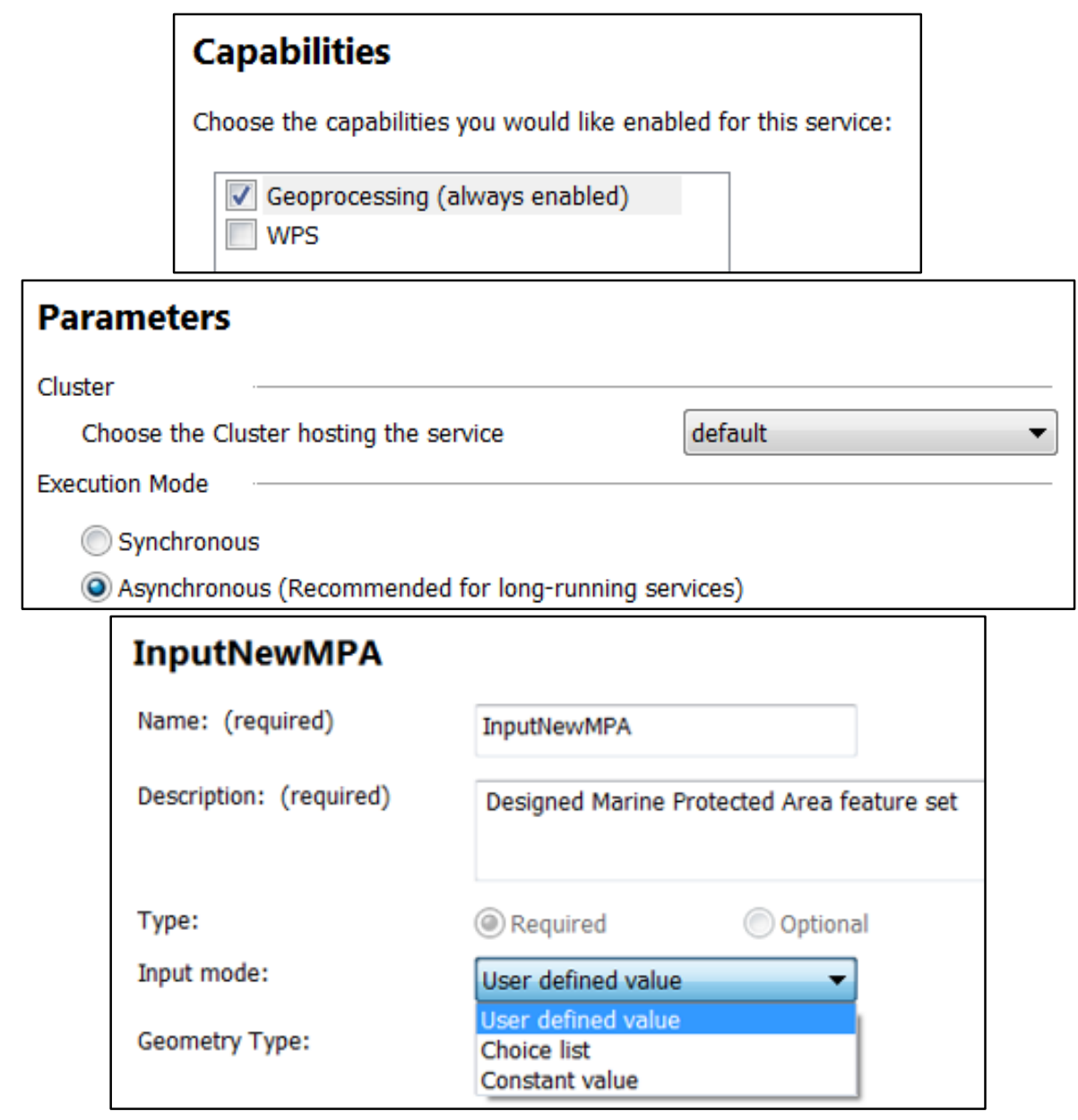

Figure 5-2: Publishing the PCMBR Geoprocessing Service 


\subsubsection{Depth Distribution of an MPA}

The depth distribution of an MPA (DDMPA) geoprocessing tool calculates depth distribution within an MPA polygon. It also was a Python script in an ArcGIS 10.3 Toolbox and had two input parameters: the Designed MPA and the Depth Zone feature classes. Figure 5-3 illustrates the program flow chart of this geoprocessing tool.

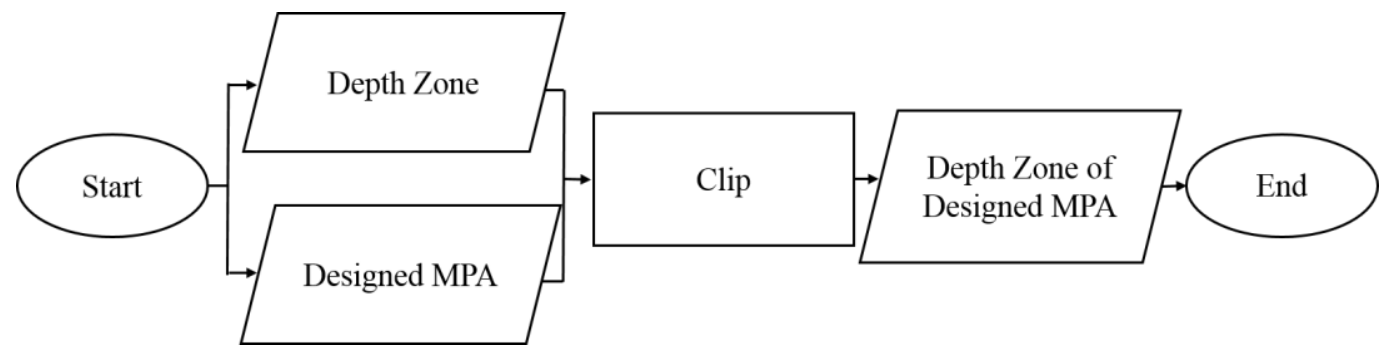

Figure 5-3: Program Flow Chart of the DDMPA Geoprocessing Tool

Regarding an MPA polygon, the DDMPA geoprocessing tool could use the Clip tool to calculate the area of depth zones within the MPA. Its output contained the three records which represented three depth zones individually. Each record had depth range and the area. The area unit was a square kilometer.

After the DDMPA geoprocessing tool was executed successfully in ArcMap 10.3, its result was shared as a DDMPA geoprocessing service. The settings for publishing this service are similar to the PCMBR geoprocessing service except for the input mode. The Designed MPA feature class had "User defined value" mode and the Depth Zone feature class had "Constant value" mode. The DDMPA geoprocessing service had geoprocessing capability and it ran as an asynchronous mode. The Designed MPA feature class was the task parameter when the service was published.

\subsection{Map Services and Feature Services}

For data visualization and editing in the web application, two types of web services were published with ArcGIS for Server: the map service and the feature service. The MPA feature class was published as a map service and a feature service. The web application consumed the map service for the viewing function and the feature service for the editing function. Although the feature service could be added as a FeatureLayer to show the data on the map, the MPA feature class has 12,520 polygons, and many polygons have a large number of vertexes. Using the feature service to show the data on the map would slow down the response of the web application and some features could not be drawn since it took so long that the request might be canceled by the server. So publishing the MPA feature class as a map service and adding it as a DynamicMapServiceLayer on the map could speed up the data loading and avoid data loss. Before publishing the service, MPA feature class had to be loaded in ArcMap 10.3. Figure 5-4 shows the settings for publishing the MPA map and feature services. 


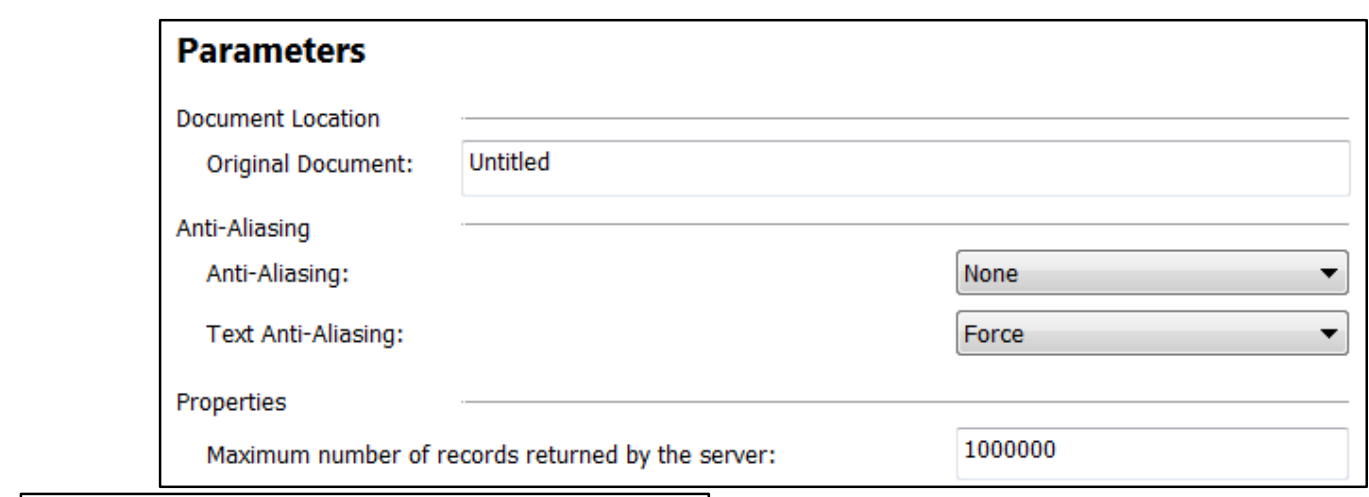

Capabilities
Choose the capabilities you would like enabled for this service:
\begin{tabular}{|l|}
\hline$\square$ Mapping (always enabled) \\
$\square$ Schematics \\
$\square$ wCS \\
$\square$ WFS \\
$\square$ Network Analysis \\
$\square$ KML \\
$\square$ WMS \\
$\square$ Feature Access \\
$\square$ Mobile Data Access \\
\hline
\end{tabular}

\section{Mapping}

REST URL: $\quad$ https://msgis-wet

SOAP URL: $\quad$ https://msgis-wet

Operations allowed:

$\checkmark$ Data $\square$ Map $\square$ Query

\section{Caching}

Draw this map service:

Dynamically from the data

Using tiles from a cache

\begin{tabular}{|l}
\hline Feature Access \\
REST URL: $\quad$ https://msgis-webdev-1.redlands.edu/arcgis/rest/service \\
SOAP URL: $\quad$ https://msgis-webdev-1.redlands.edu/arcgis/services/qia \\
Operations allowed: \\
$\square$ Create $\square$ Delete $\square$ Extract $\quad \square$ Query $\quad \square$ sync $\quad \square$ Update \\
\hline
\end{tabular}

Figure 5-4: Publishing the MPA Map and Feature Services

Since the MPA feature class had 12,520 features and there would be more MPAs proposed, the maximum number of records returned by the server needed to be set greater than the default, which was 1,000. The service had "Mapping" and "Feature Access" capabilities. Mapping settings activate all capabilities, including "Data," "Map," and "Query." The REST URL under "Mapping" is the map service's URL. The map service was drawn dynamically from the data which can show timely maps. ArcGIS for Server generates map images once the service is requested. Although the dynamic map service draws maps more slowly than a cached service, the dynamic map service is more suitable for the web application since the MPA feature service need frequent updates. Feature access capability allowed "Create," "Delete," "Query," and "Update" operations. The REST URL under "Feature Access" is the feature service's URL.

The MBR feature class was also published as a map service for viewing and a feature service for editing. The process of publishing the MBR map service and feature 
service was similar to the process of publishing the MPA map service and feature service. The difference between them was that the MBR map and feature service did not need to change the maximum number of records returned by the server. Because the MBR feature class was clipped by the high-resolution coastline, its polygons have complex boundaries with a very large number of vertexes. It can be slow in loading the feature layer in a web application.

The designed MPA feature class was published as a feature service. Since there were not many polygon features in this feature class, using the feature service for visualization would not slow down the data loading in the application.

\subsection{Web Application}

Since many marine institutes and related organizations need to collaborate when they propose a new MPA, a web application could make the proposal process more efficient. The web application was developed using Hypertext Markup Language (HTML), Cascading Style Sheets (CSS), and JavaScript. The charts and tables inside the web application were developed using Google Chart.

\subsubsection{Interface Design}

The user interface of the web application was designed to make the app easy to use. Figure 5-5 shows the initial interface of the web application. In the middle of the page, there is a map to view the MPA, MBR, and Designed MPA data. The map legends are shown at the bottom left corner of the map. It shows only the legends of the data which are added to the map. Zoom in and zoom out widgets are at the upper left corner. They can be used to change the extent of viewing. It has four panels on the right side to streamline the four steps to propose a new MPA.
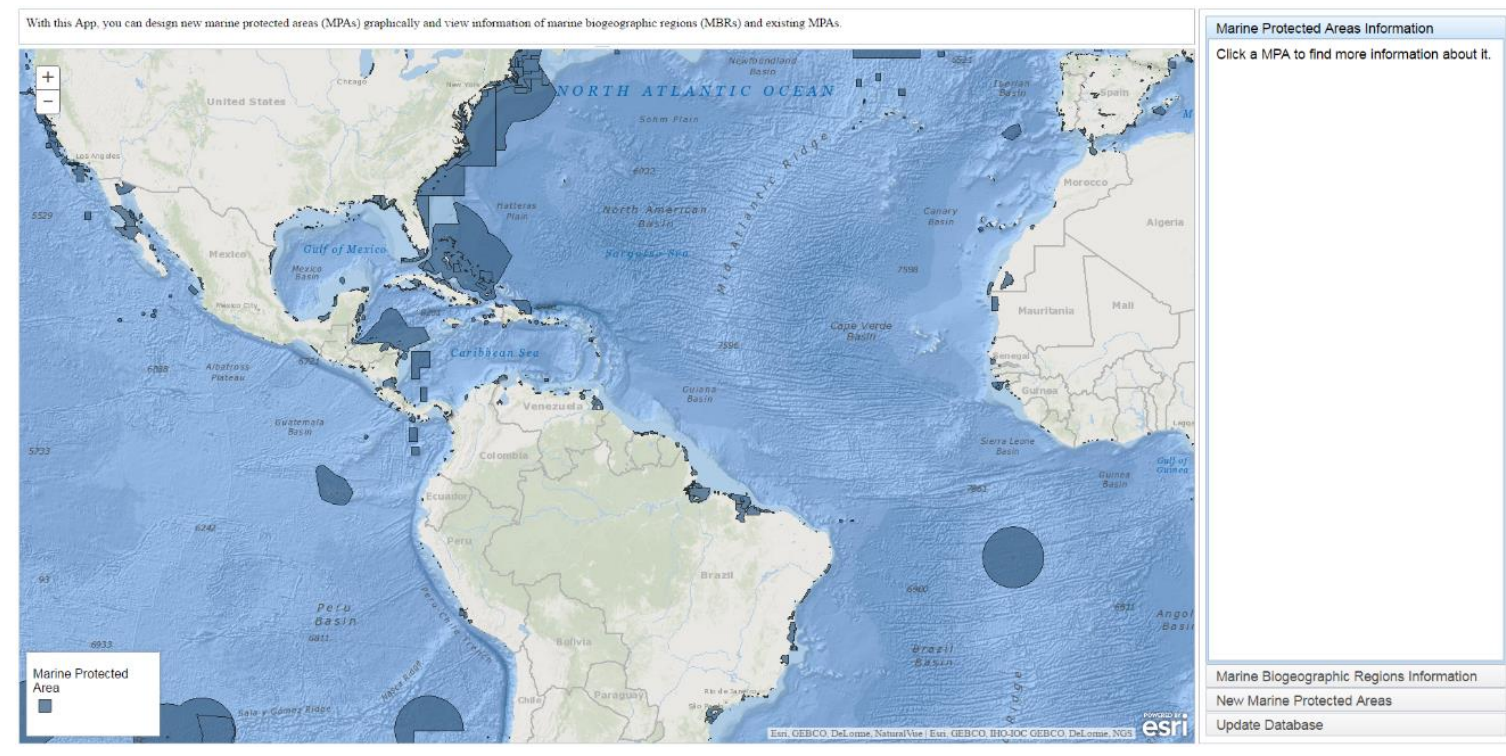

Figure 5-5: Web Application Interface 


\subsubsection{Marine Protected Areas Information}

The first panel is the initial interface shown in Figure 5-5. In this panel, the information of an MPA can be queried by clicking the MPA on the map. It includes the MPA's name, the percentage of no-take and restrictive, and the percentage of depth zones. They are displayed in the panel and the percentage data were graphically shown in the Google pie charts which can help users understand the data more intuitively. Figure 5-6 illustrates the program flow chart of this panel.

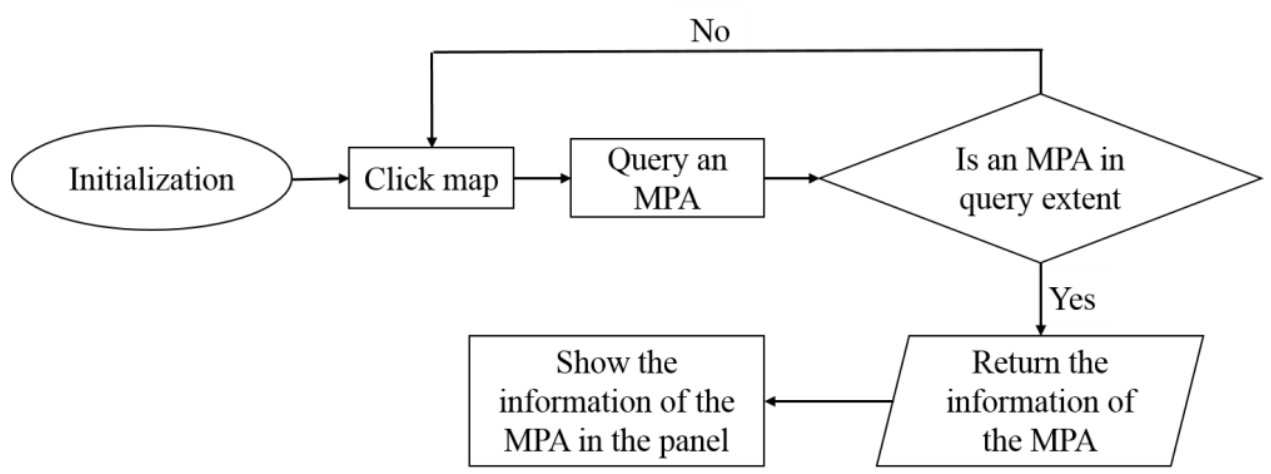

Figure 5-6: Program Flow Chart of the MPAs Information Panel

When the first panel is clicked, it is activated and initialized. Only the MPA polygons were shown as a DynamicMapServiceLayer on the map. After initializing, when the map is clicked, a query extent is built around the clicked point. ArcGIS for Server conducted the query task on the MPA map service. If there is an MPA intersect with the extent, the MPA's name, total area, the area of the no-take level, and the area of three depth zones are returned. The data are calculated as percentages and shown in Google pie charts in the panel. If there is no match, the user can still click the map to query an MPA.

\subsubsection{Marine Biogeographic Regions Information}

The information of an MBR can be queried and shown in the second panel when the MBR is clicked. When the second panel is activated, the interface of the web application is shown in Figure 5-7. 

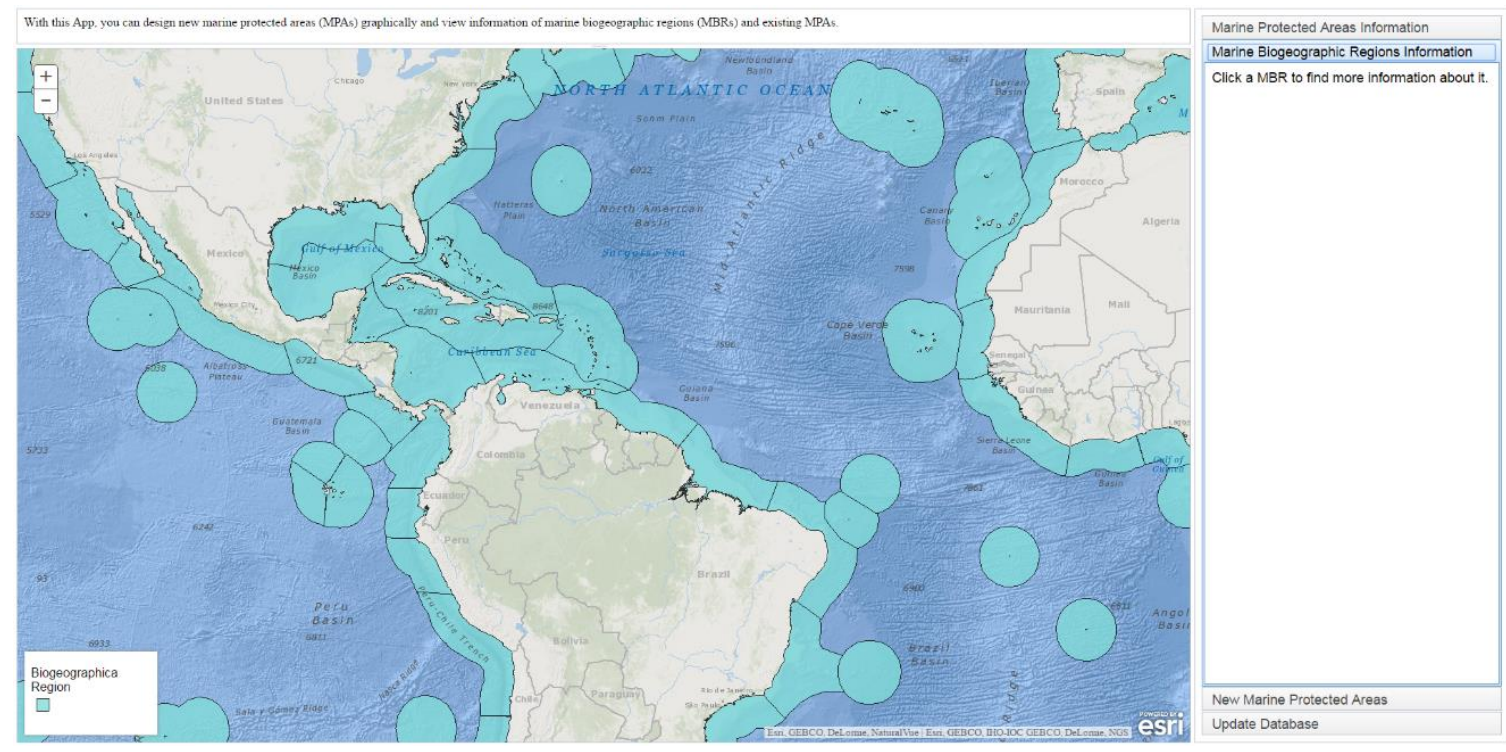

Figure 5-7: Interface of MBRs Information Panel

It contains the MBR's name, the realm, the province which the MBR belongs to, the protection percentage, the percentage of depth zones in the MBR, and the protection percentage of different depth zones within the MBR. The last two percentage data are graphically displayed in a pie chart and a bar chart to make them more visual. Figure 5-8 gives the program flow chart of this panel.

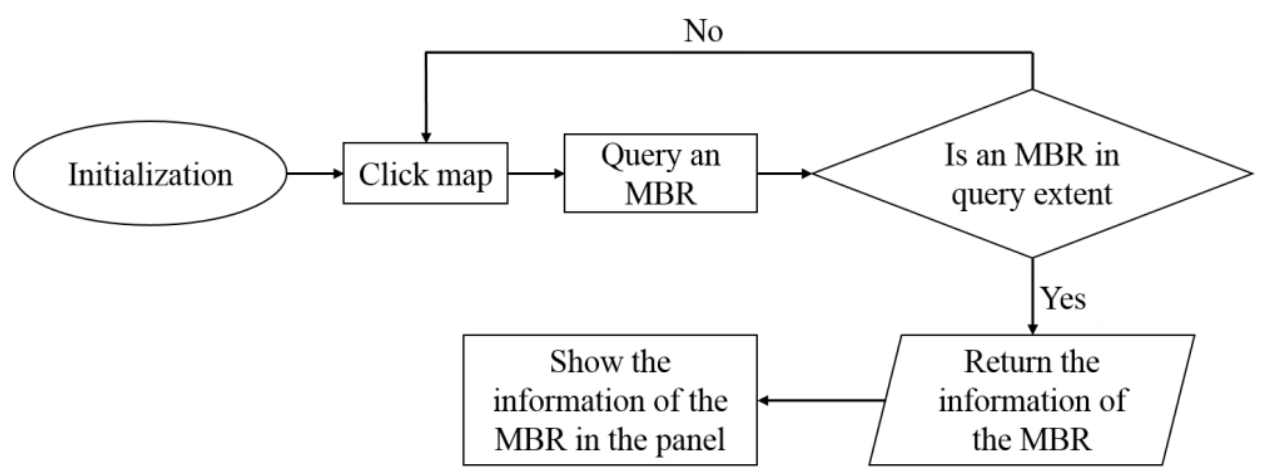

\section{Figure 5-8: Program Flow Chart of the MBRs Information Panel}

When the second panel is shown, MBR's map service is added as a DynamicMapServiceLayer on the map. The query method was the same as MPAs' in the first panel. If an MBR was queried, its name, realm, province, total area, area of three depth zones, and protected area of three depth zones would be returned. The area was calculated in percentage and shown by Google pie and bar charts in the panel. 


\subsubsection{New Marine Protected Areas}

In the third panel, a user can graphically design a new MPA and update it to the Designed MPA feature service. After drawing a new MPA, a user can examine the protection coverage of related MBRs to balance their protection during the design process. These capabilities can be implemented individually by three buttons in this panel: New MPA, Calculate, and Save MPA. Figure 5-9 displays the interface of the web application when this panel is activated. MPA, MBR, Designed MPA, and a graphic layer are loaded on the map.
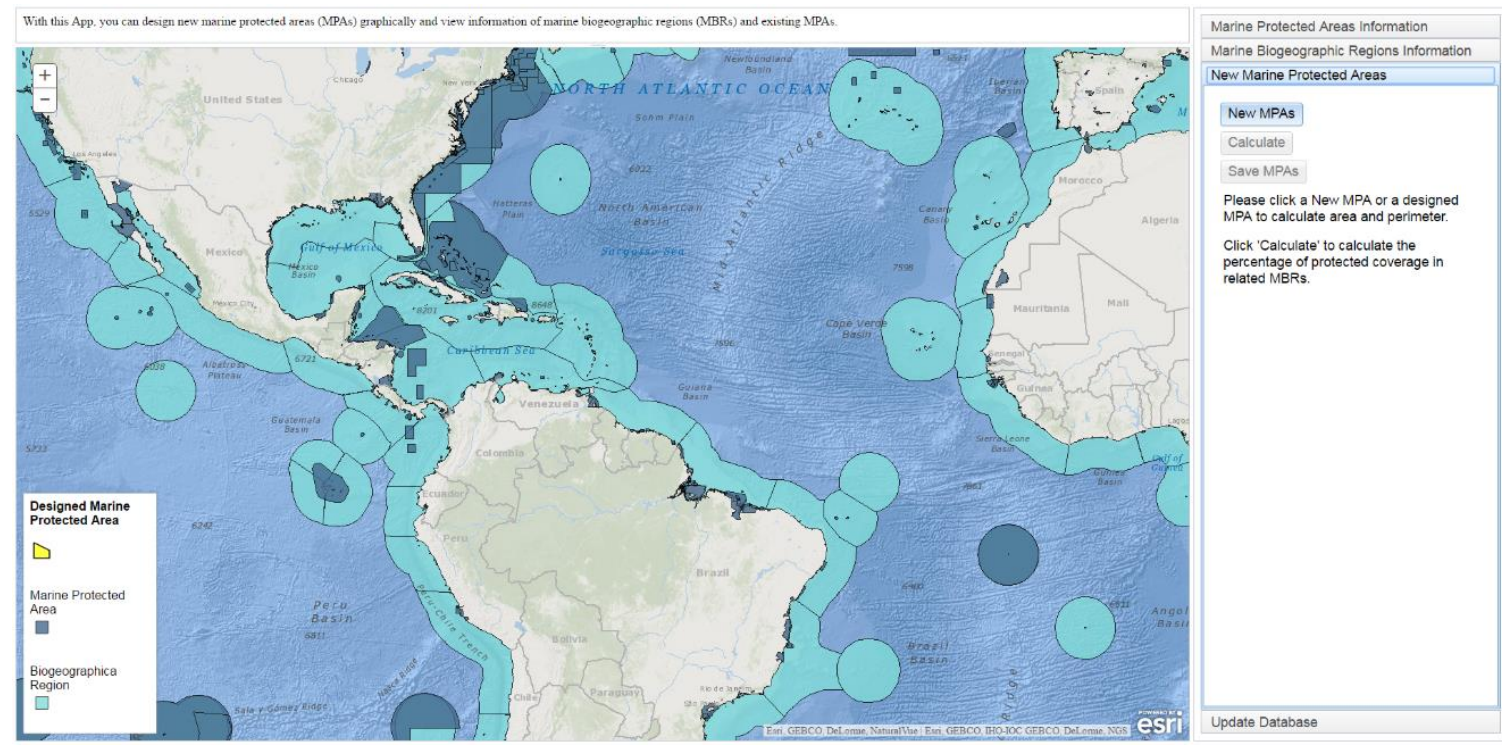

Figure 5-9: Interface of the New MPAs Panel

MPA and MBR are added as DynamicMapServiceLayers. Designed MPA FeatureLayer is from a feature service. The graphic layer is used to hold the graphics drawn by a user temporarily for designing an MPA. Before the graphics are updated to the Designed MPA feature service, they cannot be seen by others so that other people cannot interfere with the process of design. When the web application is closed, the graphic layer cannot save the user's work. If the user updates the graphic of the MPA in design to the Designed MPA feature service, the user can change it later and other users can also edit it.

The reason for updating the MPA in design to the Designed MPA instead of the MPA feature service was that the process of MPA design needed many people and organizations involved. Before an MPA was approved to update to the MPA feature service, it could be saved in an intermediate feature service - the Designed MPA. For security purposes, it would help the MPA feature service remain clean and accurate. A user could not edit existing MPA feature by mistake. At the same time, the Designed MPA feature service could save the user's work and other people can make improvements based on their work. Figure 5-10 displays the program flow chart of this panel. 


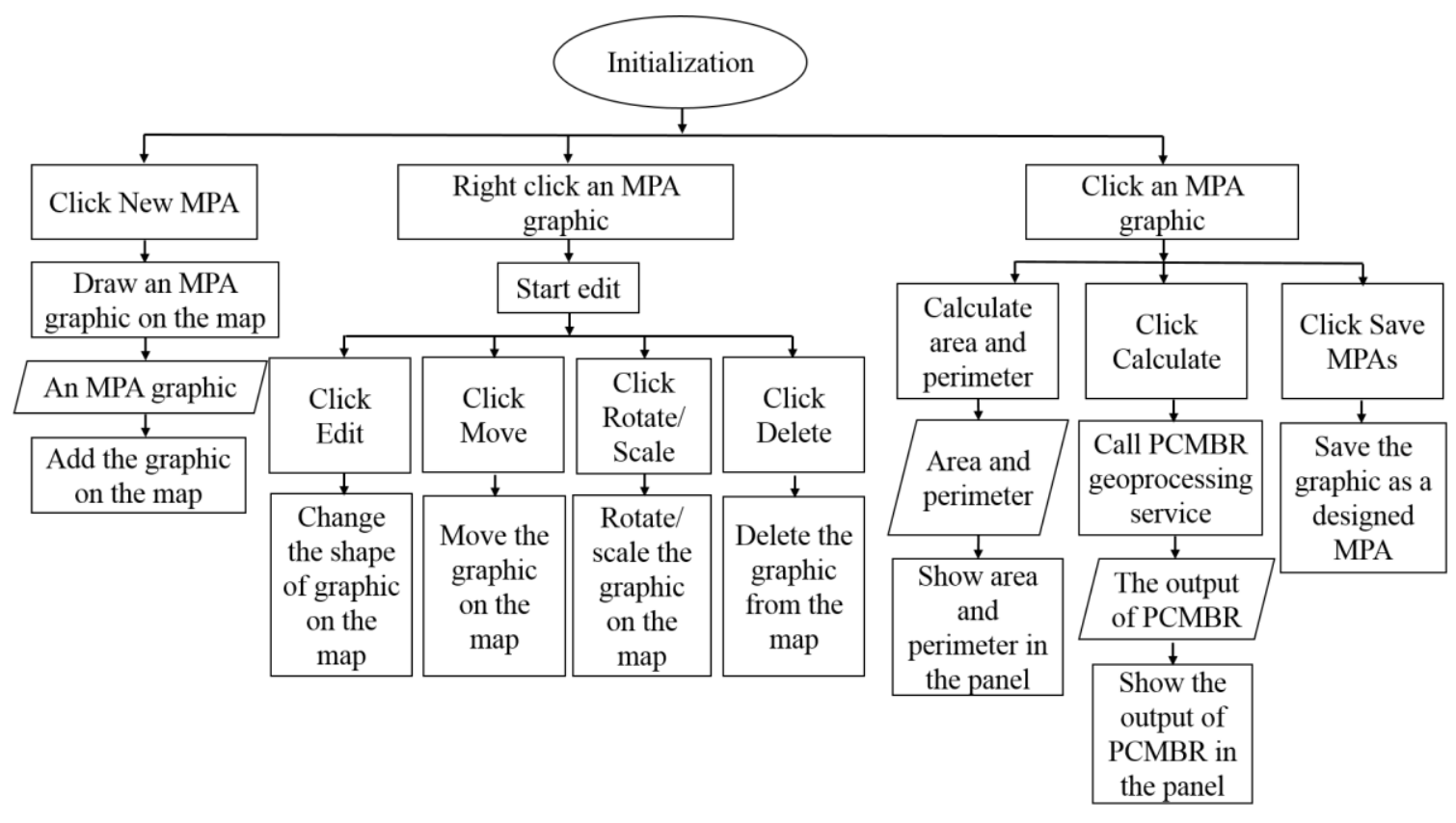

Figure 5-10: Program Flow Chart of the New MPAs Panel

There are three buttons inside the panel. The first is New MPA. When it is clicked, the draw() function of the ArcGIS API for JavaScript is activated, and a user can draw an MPA in design on the map. When done, the user can right click on the graphic to activate the edit() of the ArcGIS API for JavaScript. The application displays a menu which includes Edit, Move, Rotate/Scale, and Delete. Edit.EDIT_VERTICES, Edit.MOVE, Edit.ROTATE, and Edit.SCALE are used to change the graphic's shape, position, and size. The graphicsLayer.remove function is used to delete the graphic. When a graphic is clicked, the application called the geometryEngine.geodesicArea and geometryEngine.geodesicLength to calculate its area and perimeter. Since the study region was global, using geodesicArea and geodesicLength methods to consider the curvature of the earth yielded more accurate results.

The second button in this panel is Calculate. An MPA in design, it is used to calculate the percentage of related MBRs protected by this MPA and existing MPAs when a graphic is selected. A user can apply these data to balance their protection when an MPA is in design. If an MPA in design is clicked, this button is enabled. When the Calculate is clicked, the application calls the PCMBR geoprocessing service and receives the outputs from the GIS server. The application shows the job status of this geoprocessing service to let users monitor the process. The output area data are calculated and shown as percentages. The data of each MBR are displayed in a Google table individually, which are easier for users to understand and visualize.

The last button in this panel is the Save MPA button. When a graphic is selected, it was enabled. It is used to update a graphic to the Designed MPA feature service with FeatureLayer.applyEdits(). The Designed MPA feature class has the same schema as the MPA feature class. It holds the MPA candidates to help keep the MPA database clean and make the process of design rigorous. 


\subsubsection{Update Database}

The last panel of the web application is Update Database. In this panel, a user can change and update the attributes of a designed MPA. If the designed MPA is approved, it can be updated to the MPA database and the related MBR protection information are updated. Figure 5-11 displays the interface when the last panel is clicked. When this panel is shown, MPA, MBR, and Designed MPA layers are loaded. MPA and MBR are added as DynamicMapServiceLayers. Designed MPA are added as a FeatureLayer.

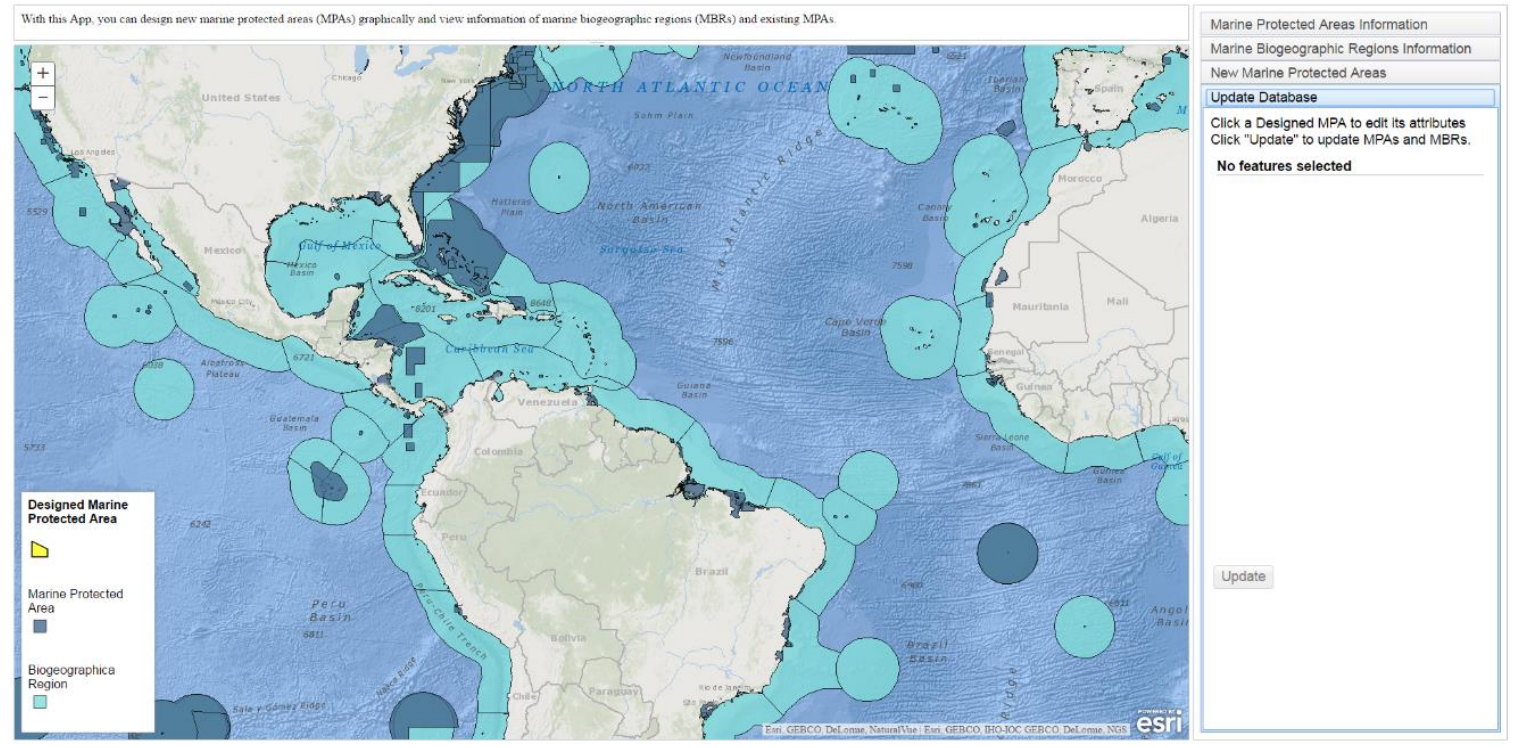

\section{Figure 5-11: Interface of the Update Database Panel}

Figure 5-12 illustrates the program flow chart of the Update Database panel. When a designed MPA is clicked, the application called Attribute Inspector located in the panel appears to let a user type the attributes of the designed MPA. The Save button is used to save the attributes. When the key attributes (Name and ID) are empty, the web application shows a warning message to inform users to input the attributes. At the same time, when Save is clicked, the application calls the DDMPA geoprocessing service and receives the outputs of DDMPA from the GIS server. The outputs are the distribution of depth zones in the selected MPA. The Delete button is used to delete the selected MPA. 


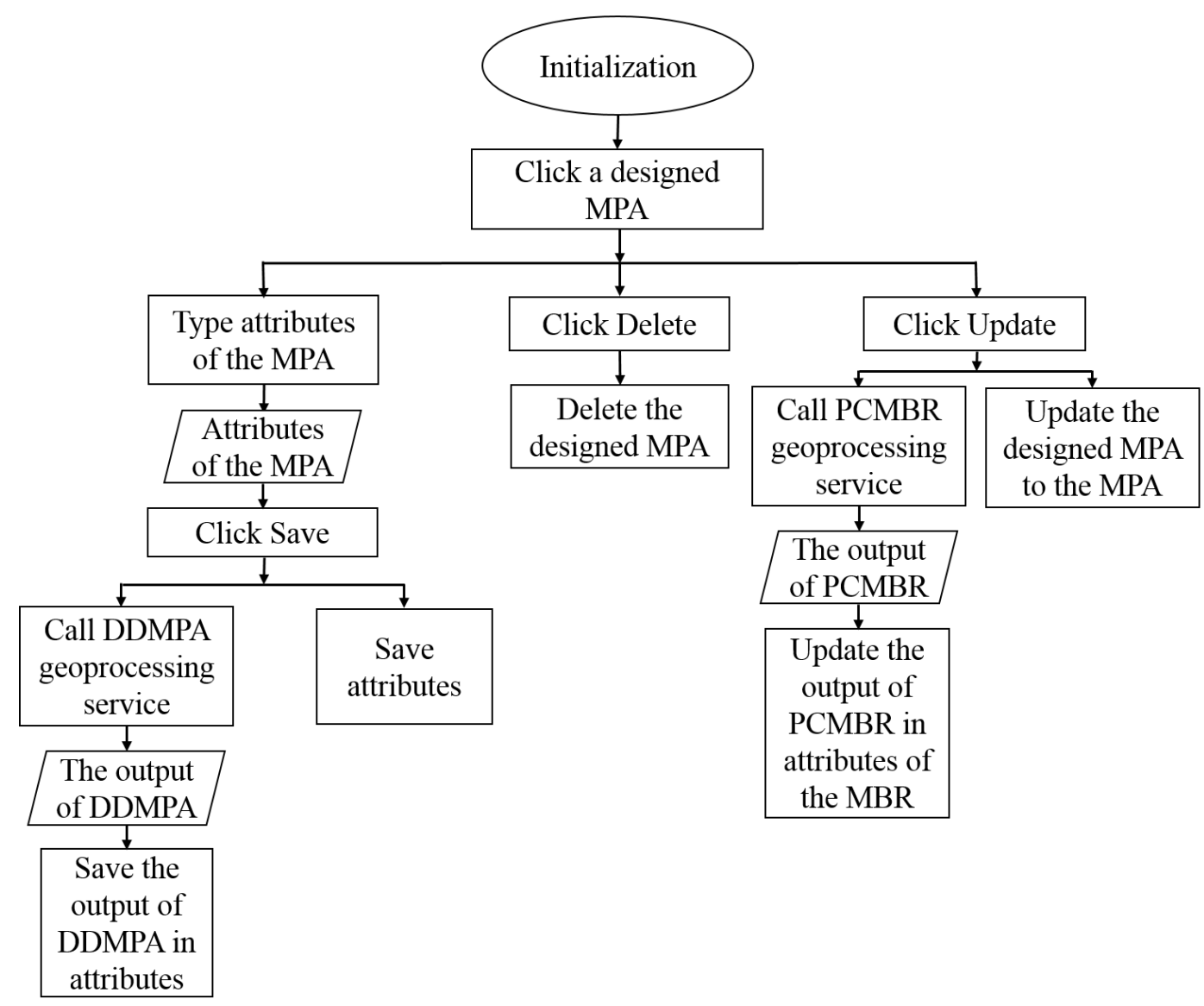

\section{Figure 5-12: Program Flow Chart of the Update Database Panel}

Because MPAs and MBRs must be edited, they also are published as the feature services but not added to the map. When a designed MPA is selected, a user can click the Update button to update the selected designed MPA to the MPA feature service. At the same time, the application calls the PCMBR geoprocessing service and receives the outputs from the GIS server. Then the application updates the outputs to the protected area attributes of the MBR feature service. If the designed MPA does not have key attributes when Update is clicked, the web application shows an error message to inform the user to define the attributes. All the edited data are sent to the GIS server with FeatureLayer.applyEdits().

\subsection{Summary}

This chapter discussed in detail how the geoprocessing services and the web application were developed. Section 5.1 described the programming algorithm and publishing processes of the two geoprocessing services. Section 5.2 stated the publishing processes of the map services and feature services. Section 5.3 illustrated the interface design of the web application. It also discussed the programming logic and implementations of the four panels which were contained by the web application. 



\section{Chapter 6 - Use Cases}

The main purpose of this project was to help the Marine Conservation Institute design new marine protected areas (MPAs) and help the institute collaborate with its partners efficiently to propose new MPAs. Section 6.1 covers a use case for a user who wants to learn about existing MPAs. Section 6.2 shows a use case for a user who wants to query marine biogeographic regions (MBRs) data. Section 6.3 illustrates how to design a new MPA and update it to the existing MPA feature service. Also, it shows how to update protection status information of its related MBRs.

\subsection{Use Case: Exploring Marine Protected Areas Information}

In this scenario, a user wants to browse the MPAs, is interested in the current distribution of existing MPAs, and needs essential information about them.

When the application is initialized, the Marine Protected Areas Information panel is activated. The user can explore an existing MPA by clicking the MPA on the map. The MPA layer is displayed in Figure 6-1. The user can pan to the MPA to investigate. The MPA turns dark blue once it is selected. Information shown on the right panel tells the user that the MPA's name is Great Barrier Reef. According to the first pie chart, about $32 \%$ of this MPA is protected at the no-take level, which means that no resources can be taken out of this part of the MPA. The ocean areas are different from land regions. Besides the sea surface, the user also needs the information of depth zones because species vary between water depths. The second pie chart shows that almost $70 \%$ of this area is shallower than $200 \mathrm{~m}$, and $17.2 \%$ of this area is deeper than $1,000 \mathrm{~m}$. The user also can use the mouse scroll wheel or the Zoom button to zoom in to these MPAs. 


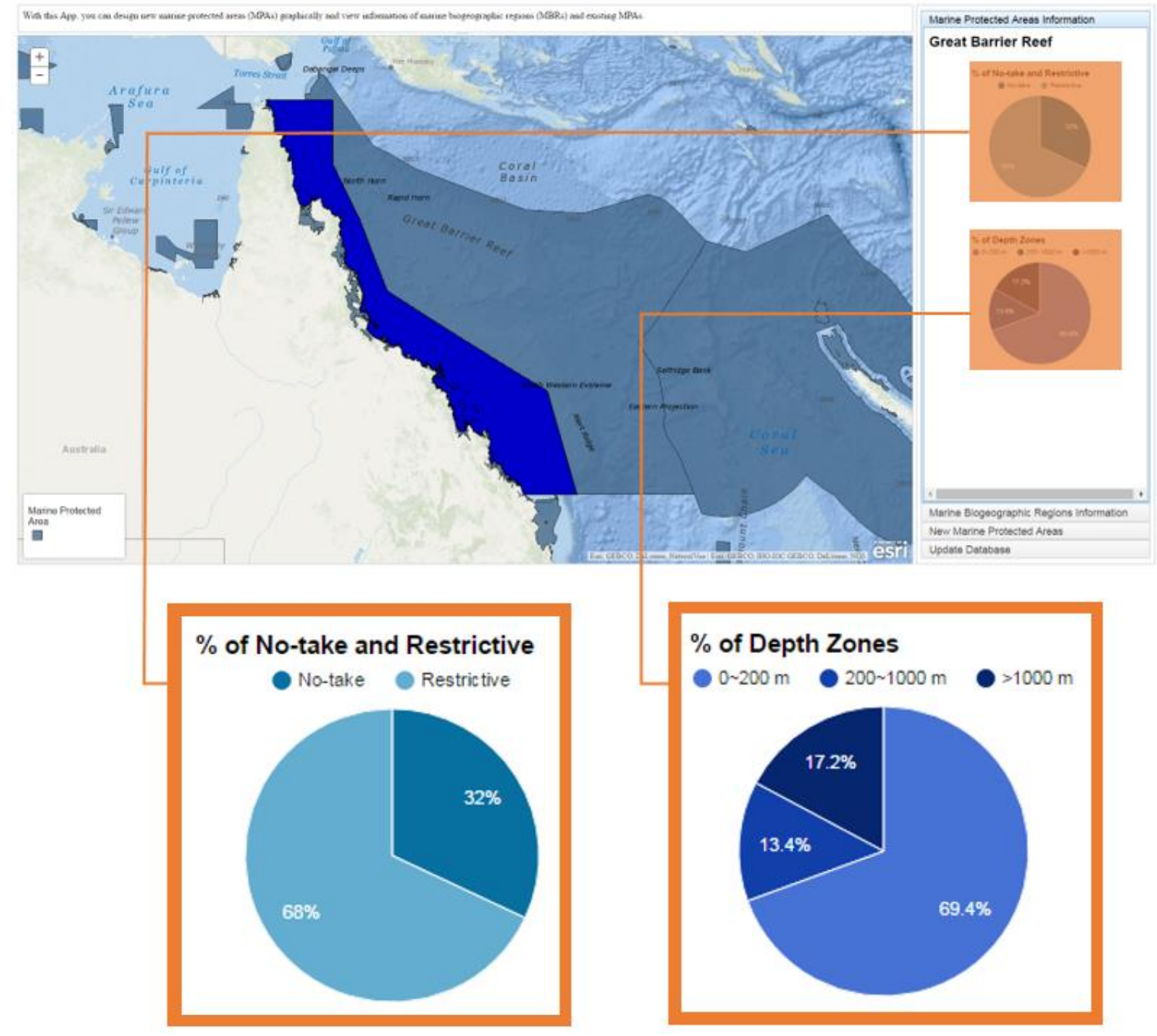

Figure 6-1: Marine Protected Areas Information Panel

\subsection{Use Case: Exploring Marine Biogeographic Regions Information}

The second scenario is to show how a user can query the MBR data. When the user goes to the second panel, the MBR layer is loaded on the map. When the user clicks an MBR, the selected region becomes dark blue too. In Figure 6-2, the Heard and Macdonald Islands region is selected and shown in dark blue. The application retrieves the records from the MBR feature service published by ArcGIS for Server and the results are output to the right panel as text and charts. The user knows that this MBR belongs to the Subantarctic Islands province and the Southern Ocean realm. About $15 \%$ of this MBR is protected by MPAs. However, it still does not reach the goal of the institute, which is that at least $20 \%$ of each MBR is protected by MPAs by 2030.

The depth distribution of this MBR is shown in the pie chart. A large part of the distribution is in the relatively deep water area. According to the bar chart, the shallow water $(<200 \mathrm{~m})$ zone is protected better than the deep water. The MBR needs more balanced protection among its three depth zones. 


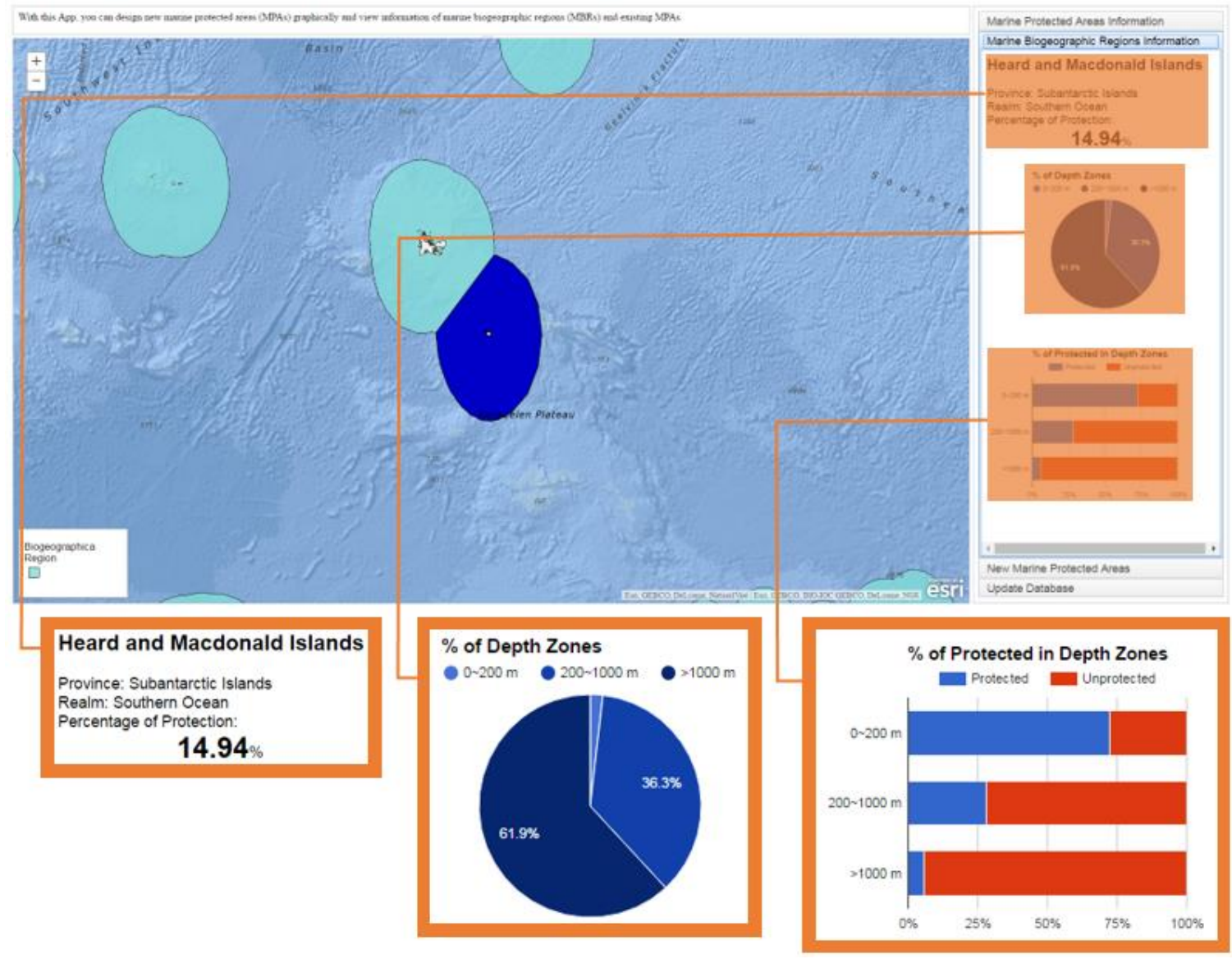

Figure 6-2: Marine Biogeographic Regions Information Panel

\subsection{Use Case: Design and Update of Marine Protected Areas}

The final scenario deals with a staff member in the institute who wants to propose a new MPA around the Philippines. The user first goes to the MBR's information panel and pans to this area. The user clicks one MBR, which is highlighted in Figure 6-3, to see its information. The user learns that this MBR is named South Kuroshio and that only $0.33 \%$ of this region is protected. Then the user selects its neighbor region, called Eastern Philippines, and sees that only $2.11 \%$ of this region is protected (Figure 6-4). 

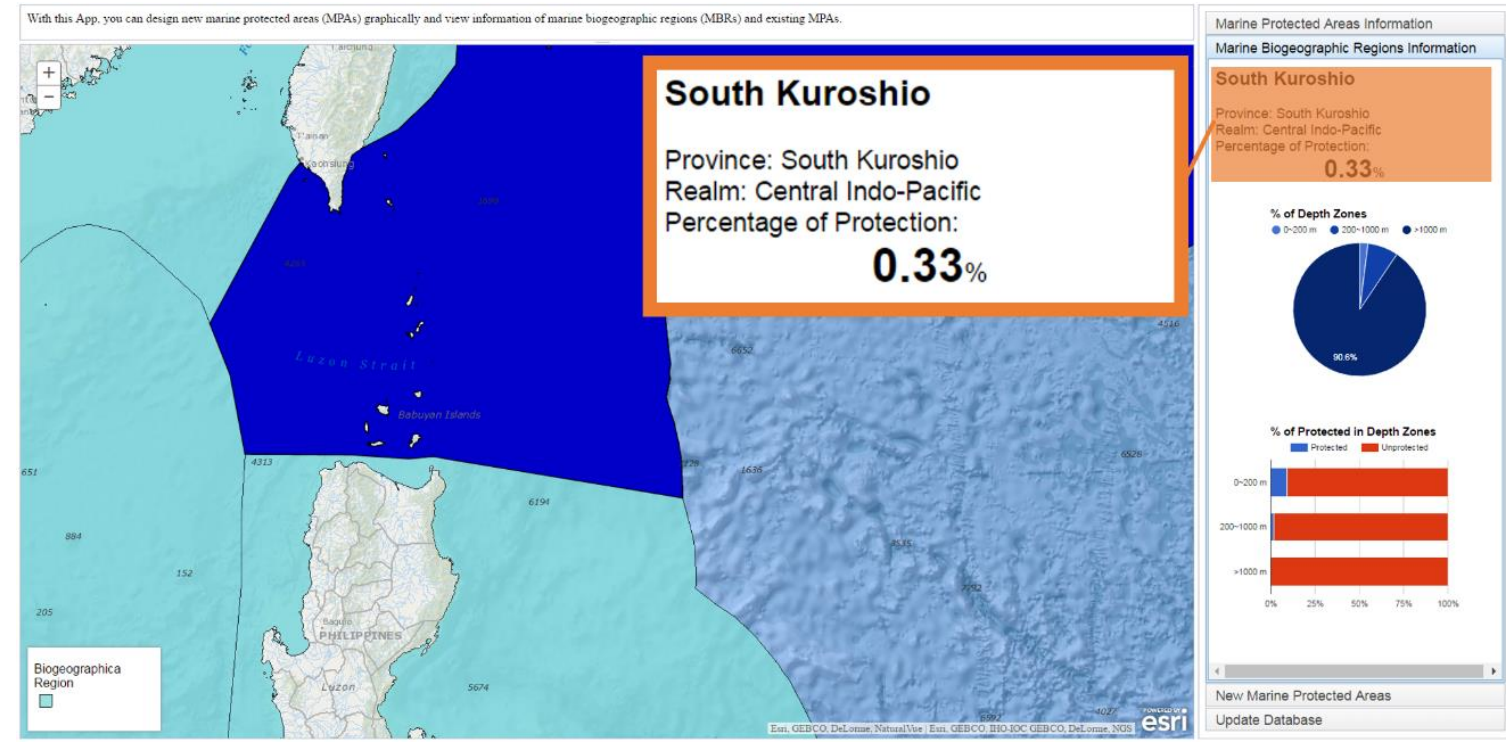

Figure 6-3: South Kuroshio Marine Biogeographic Region
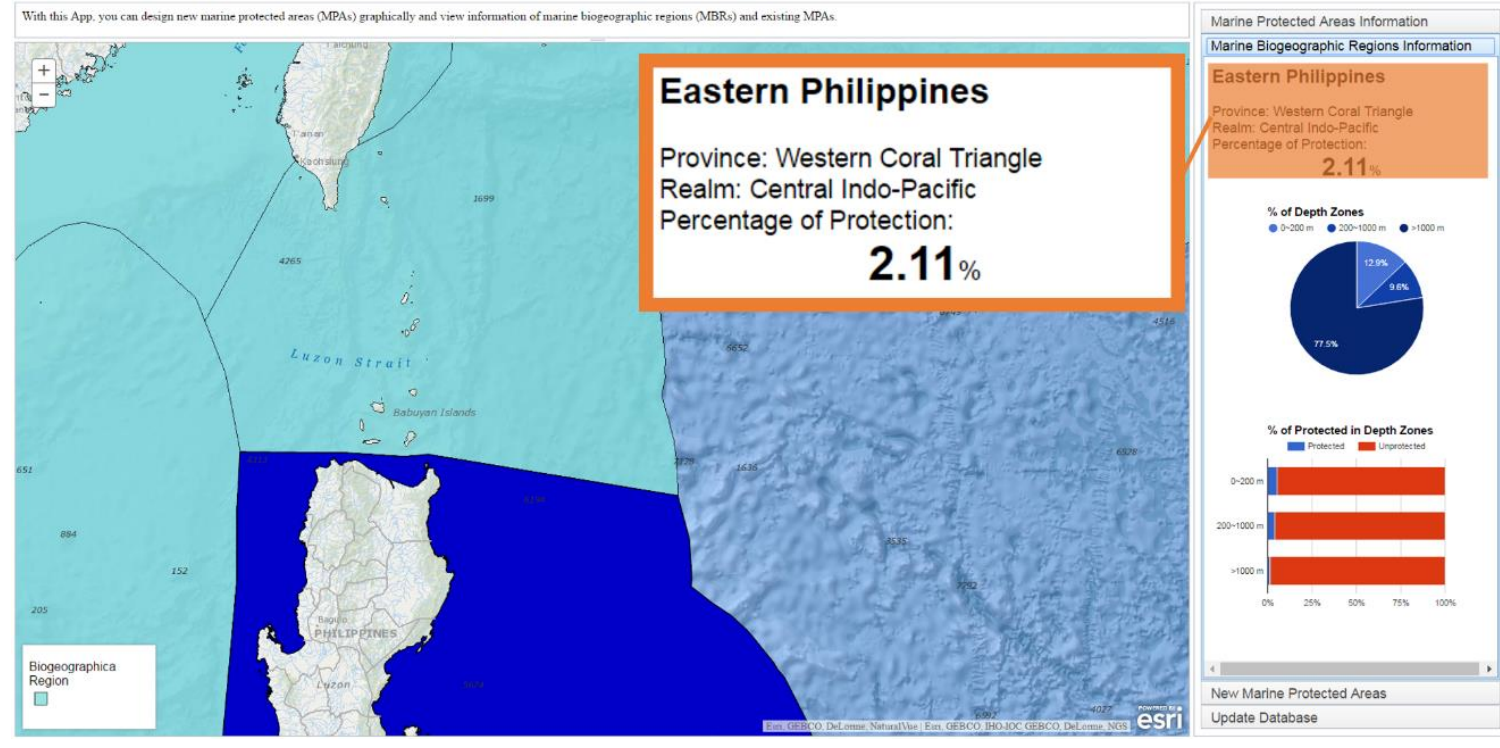

Figure 6-4: Eastern Philippines Marine Biogeographic Region

Both of these MBRs need more protection, so the user wants to design an MPA to protect them. To design a new MPA, the third panel, labelled as "New Marine Protected Area," should be used. When it is activated, the MPA, MBR, and Designed MPA layers are loaded, as shown in Figure 6-5. Starting by clicking the New MPA button, the user draws a new MPA on the map. This new MPA turns dark blue once it is selected, and its area and perimeter are shown in the right panel. 


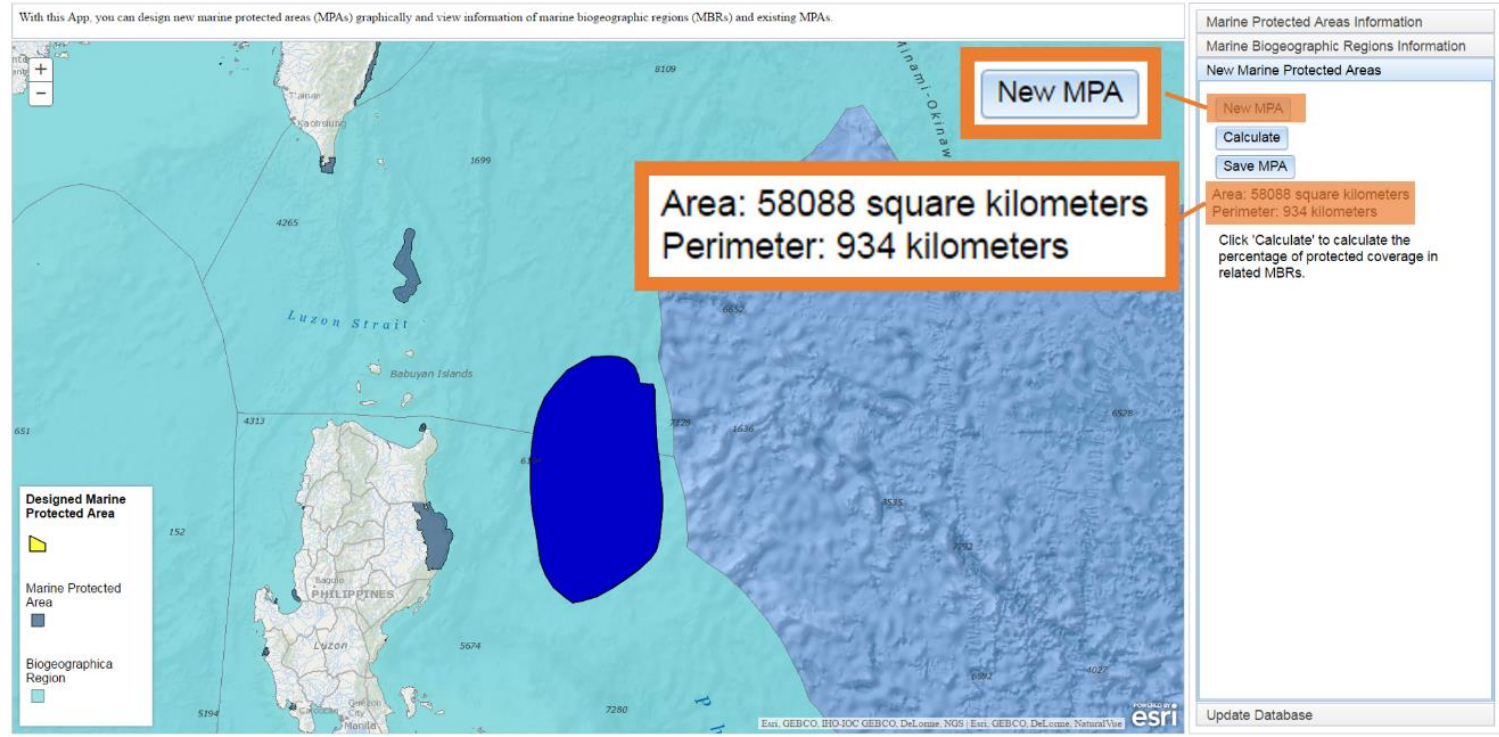

Figure 6-5: Draw New Marine Protected Areas

After the new MPA is drawn, the user wants to examine the current protection status of these two MBRs, especially how much of these MBRs is protected by this new MPA and existing MPAs. The user clicks the Calculate button, which sends the graphic of this new MPA to a geoprocessing service and retrieves the results from the GIS server. Figure 6-6 shows the results in the panel as tables. From the red boxes of these tables, the user learns that the percentage of the Eastern Philippines MBR protected by MPAs has increased from $2.106 \%$ to $6.388 \%$ and that the percentage of the South Kuroshio MBR has increased from $0.33 \%$ to $2.21 \%$. Both of them would be protected more by the new MPA. But their protection levels are not balanced. The user needs to make some changes for the new MPA to balance the protection between the MBRs.

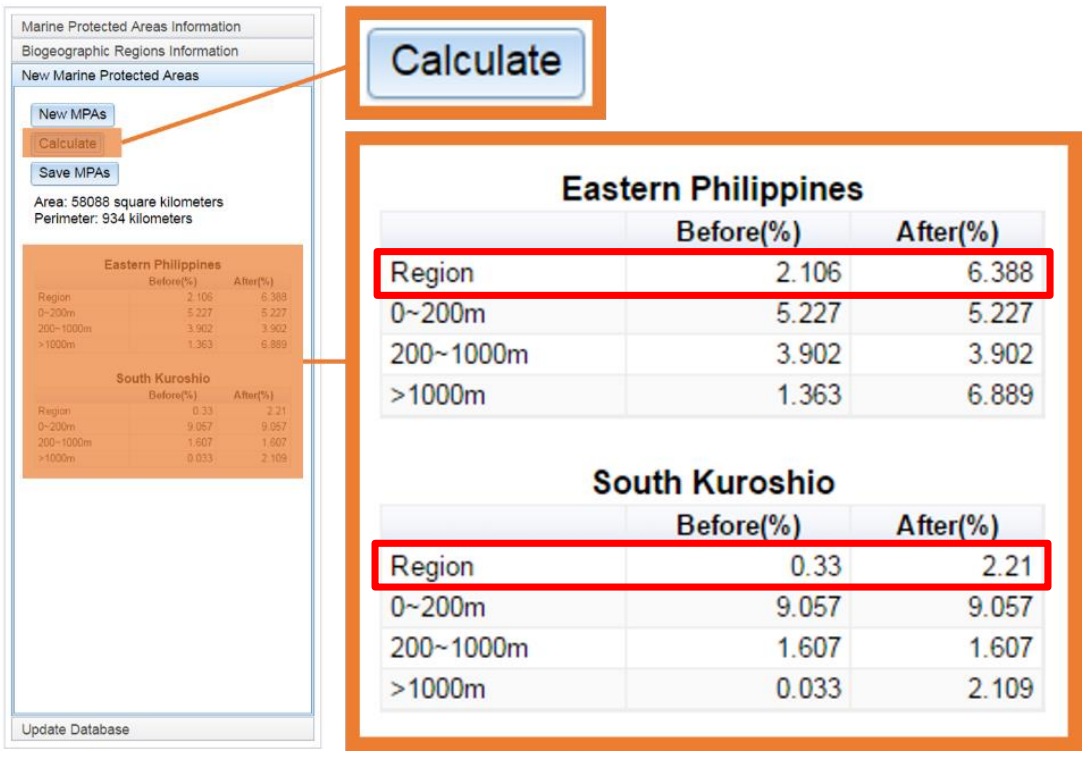

Figure 6-6: Calculate Protection Status of Marine Biogeographic Regions 
In order to modify this MPA, the user right clicks it and chooses Move from the drop down list (see Figure 6-7). After the edit function is activated, the MPA moves up to cover the South Kuroshio MBR more. Then the user clicks the Calculate button again. In the new tables that display in the panel, the red boxes (see Figure 6-7) illustrate not only that the MBRs are protected more but also that there is more balanced protection between them. For each of them, about $4.1 \%$ of the area is protected by the MPAs. To achieve further balanced protection among three depth zones in each MBR, the user could also edit this MPA's shape, size, and location based on the data shown in the tables. When the user is satisfied with this MPA, the Save MPA button (shown in Figure 6-8) is clicked to save it as an MPA in design to the Design MPA feature service. It is displayed as a yellow graphic in Figure 6-8.
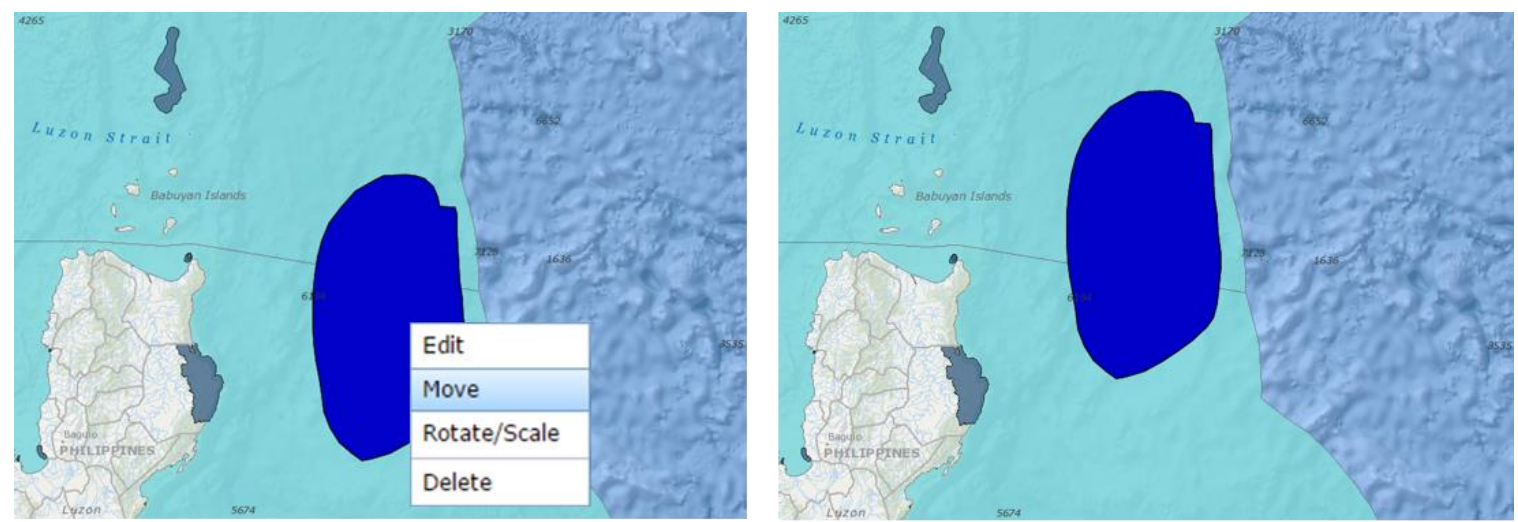

\begin{tabular}{|c|c|c|}
\hline \multicolumn{3}{|c|}{ Eastern Philippines } \\
\hline & Before(\%) & After(\%) \\
\hline Region & 2.106 & 4.138 \\
\hline $0 \sim 200 \mathrm{~m}$ & 5.227 & 5.227 \\
\hline $200 \sim 1000 \mathrm{~m}$ & 3.902 & 3.902 \\
\hline$>1000 \mathrm{~m}$ & 1.363 & 3.985 \\
\hline \multicolumn{3}{|c|}{ South Kuroshio } \\
\hline & Before(\%) & After(\%) \\
\hline Region & 0.33 & 4.191 \\
\hline $0 \sim 200 \mathrm{~m}$ & 9.057 & 9.057 \\
\hline $200 \sim 1000 \mathrm{~m}$ & 1.607 & 1.607 \\
\hline$>1000 \mathrm{~m}$ & 0.033 & 4.296 \\
\hline
\end{tabular}

Figure 6-7: Calculate New Protection Status of Marine Biogeographic Regions 


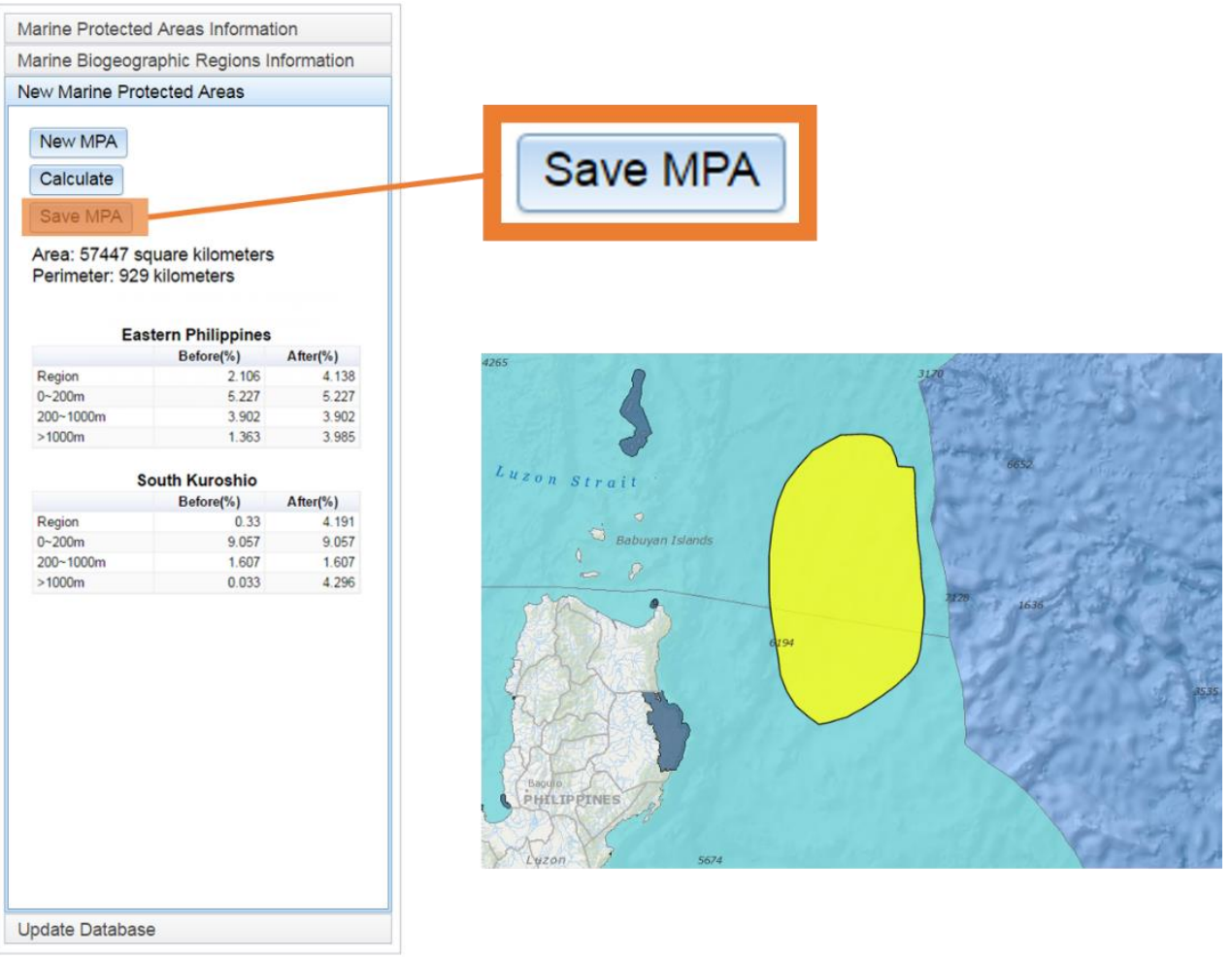

Figure 6-8: Save Marine Protected Areas

After designing the geometry of this MPA, the user goes to the last panel, Update Database, to define its attributes. The MPA, MBR, and Designed MPA layers are displayed in Figure 6-9. The user types the information (the name, ID, and so forth) and clicks the Save button. This button also calls a geoprocessing service to calculate the depth distribution of this MPA, which is saved as attributes automatically.

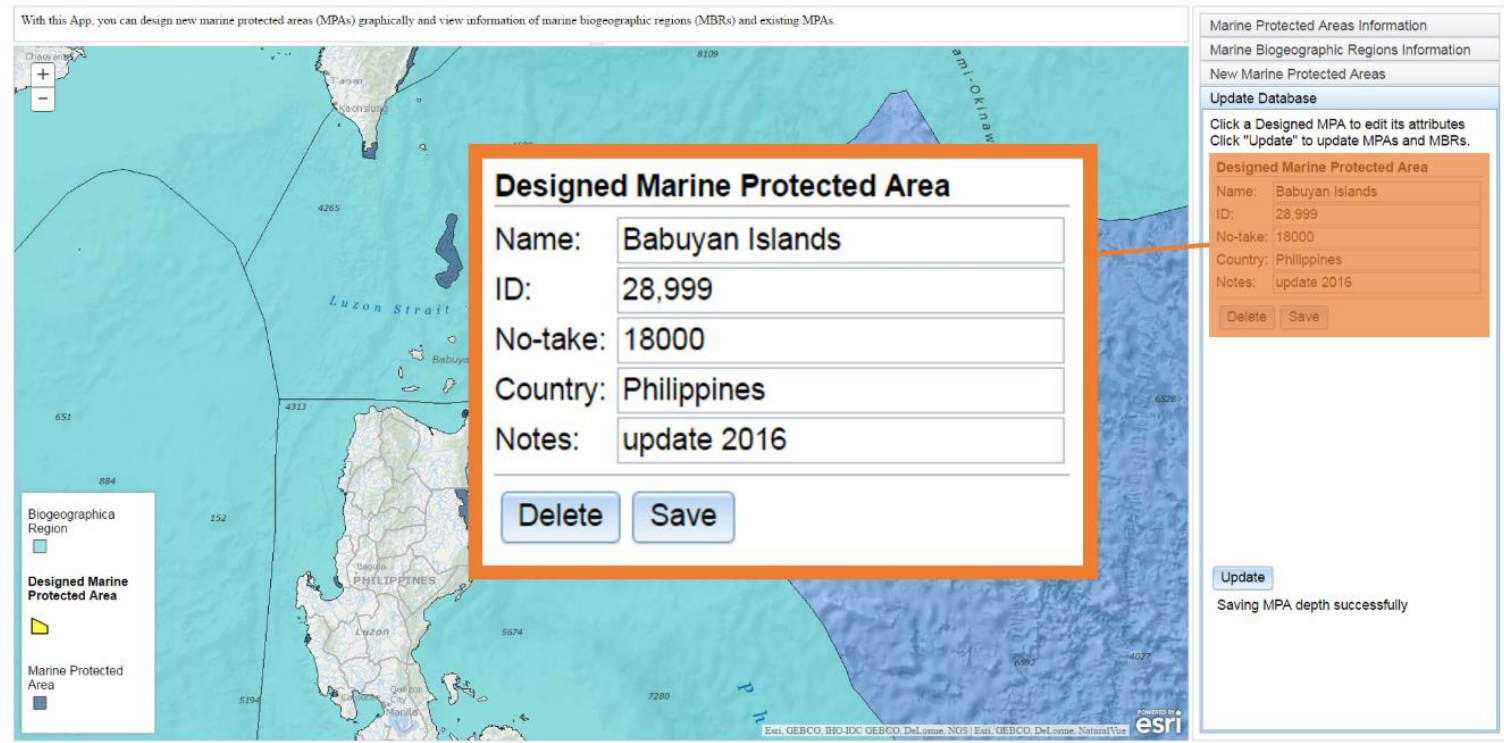

Figure 6-9: Define the Attributes of the Marine Protected Area in Design 
When this MPA is accepted by other marine institutes or related organizations, the user clicks the Update button (shown in Figure 6-10) to upload it to the existing MPA feature services. At the same time, a geoprocessing service that could calculate how much of these two related MBRs is protected by MPAs is called from the GIS server to update the protection status information about the two MBRs. After the MPA is updated, it turns blue as an existing MPA.

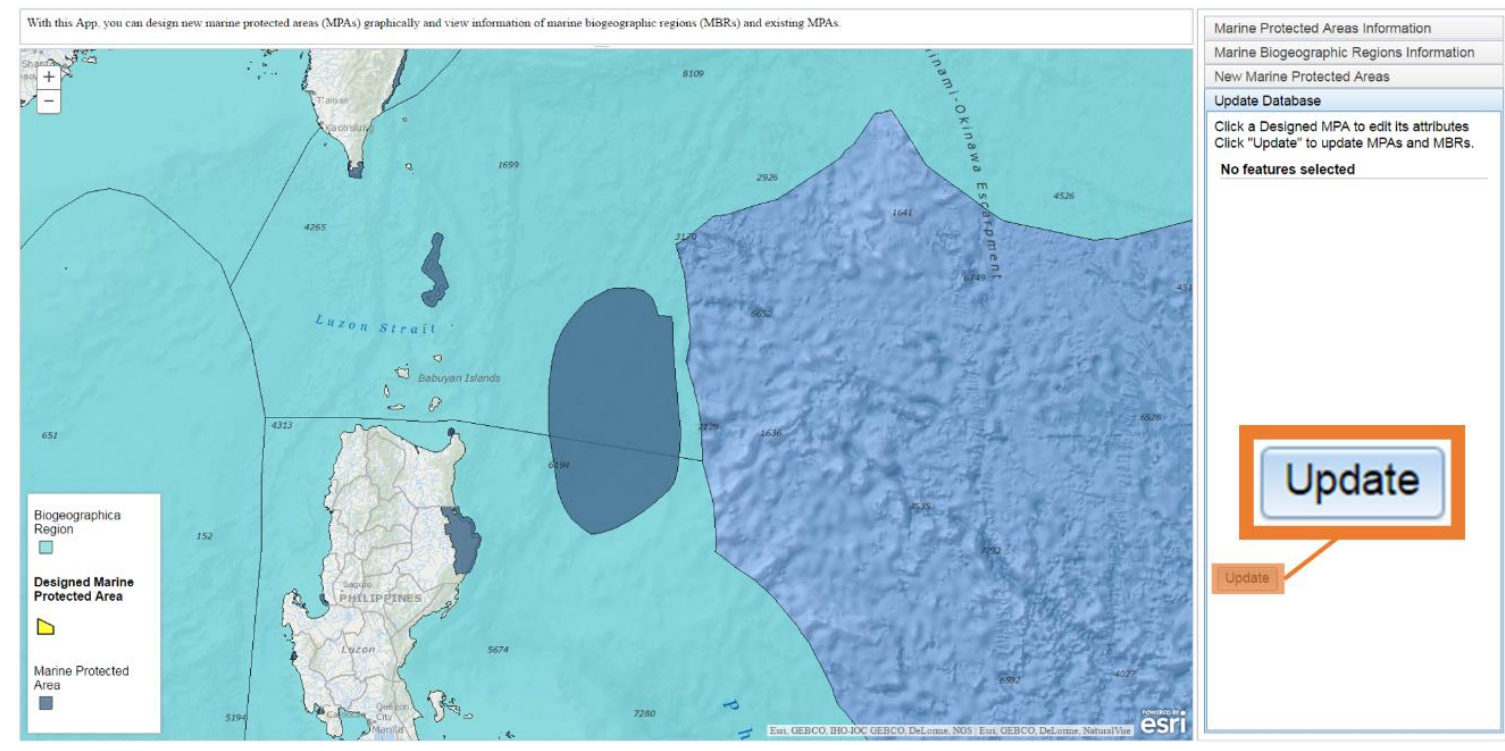

Figure 6-10: Update the Marine Protected Area in Design

The user wants to examine the updated MPA and the protection status information of its related MBRs. The user goes to the first panel and the newly created MPA is displayed. Once it is selected, its information is retrieved, as shown in Figure 6-11. About $31 \%$ of this MPA is protected at the no-take level, and all of this area is deeper than 1,000 $\mathrm{m}$. The user also clicks the second panel (Figure 6-12) to check the new protection status of the two MBRs. After the Babuyan Island MPA is updated, 4.19\% of South Kuroshio and $4.14 \%$ of Eastern Philippines are protected by the MPAs. 

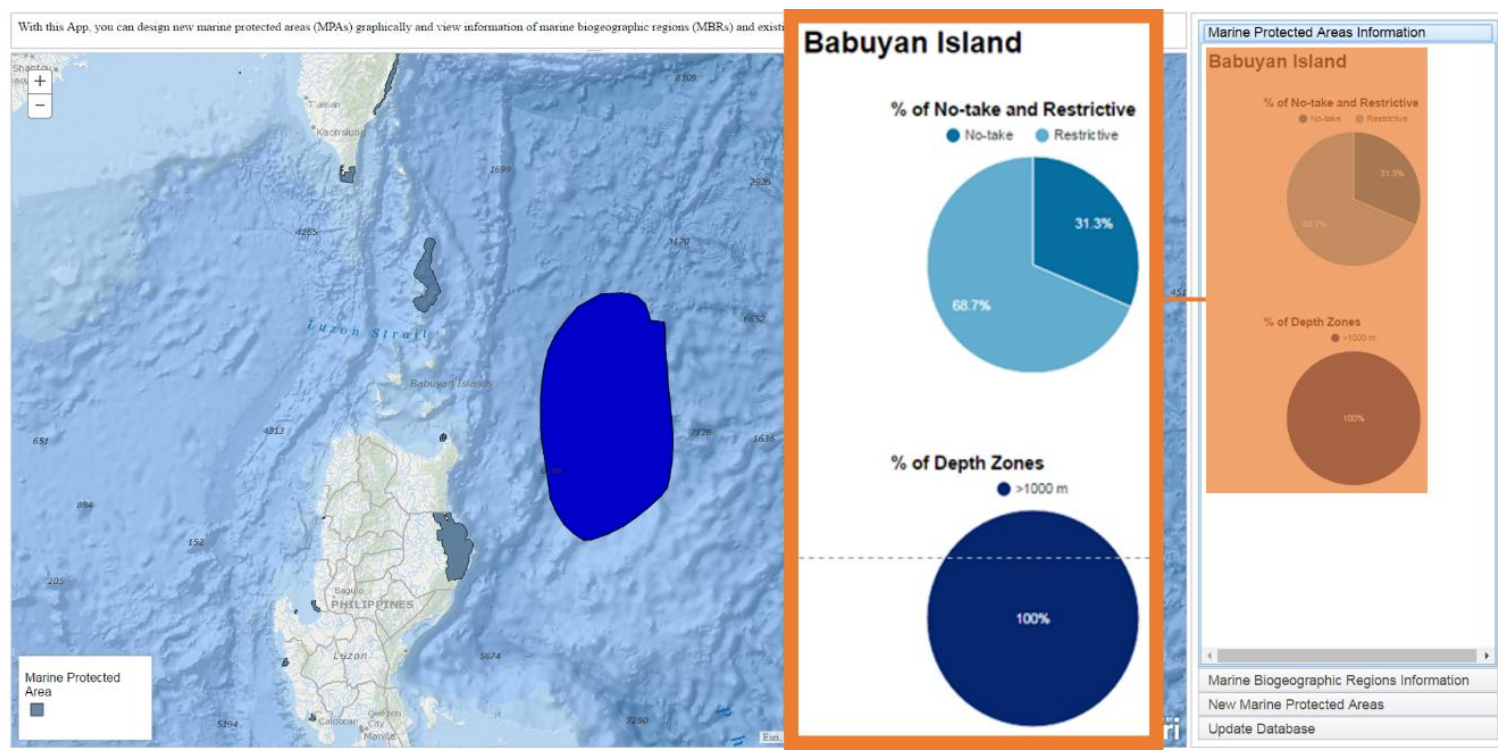

Figure 6-11: Define the Attributes of a Marine Protected Area in Design
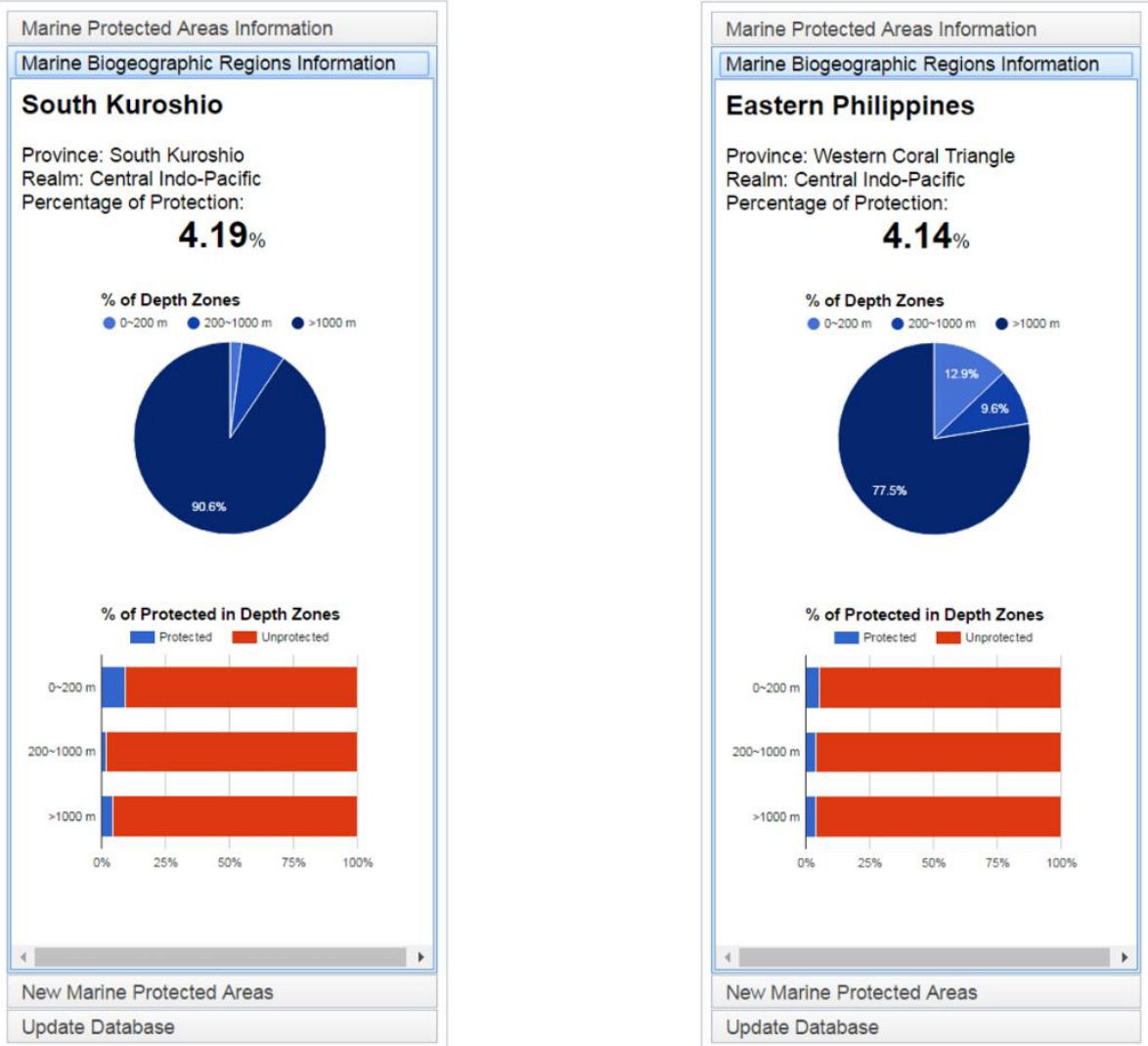

Figure 6-12: Updated Marine Biogeographic Regions Information 


\subsection{Summary}

This chapter illustrated three use cases. Section 6.1 and Section 6.2 showed how a user can explore the information of existing MPAs and MBRs. Section 6.3 illustrated the process of designing an MPA and updating the MPA and protection status information of its related MBRs. 


\section{Chapter 7 - Conclusions and Future Work}

This chapter includes two sections. Section 7.1 provides a summary of the project, and Section 7.2 presents the future work which could be added to this project.

\subsection{Project Summary}

The client for this project, the Marine Conservation Institute, needed to balance the protection coverage of the related marine biogeographic regions (MBRs) and their depth zones when the institute proposes new marine protected areas (MPAs). The institute also needed to collaborate with its partners efficiently to propose new MPAs and update them to an MPA database. To meet the requirements of the client, the project developed a web GIS application with which the client can query MPA and MBR data, graphically design a new MPA, examine the current and designed protection status of its related MBRs, and share the information with other marine organizations. After the MPA is accepted, the client also can use this application to update this MPA and the protection status information of its related MBRs.

The project preprocessed the MPA and MBR feature classes using ArcMap 10.3. Two geoprocessing tools were developed using Python. These data and tools were published as feature services, map services, and geoprocessing services with ArcGIS for Server. All these services were consumed in the web application which was developed using JavaScript, Hypertext Markup Language, and Cascading Style Sheets. It used ArcGIS API for JavaScript, the Dojo Toolkit, and Google Charts for development. The map services were used for speeding up data visualization and the feature services were used for data editing. Given an MPA, the geoprocessing services calculated the protection coverage of its related MBRs and its depth distribution. By developing the web application, the project showed how to share the information about MPAs and MBRs, and how to propose a new MPA efficiently.

\subsection{Future Work}

The project successfully demonstrated how to help the institute propose new MPAs and how to help them with their partners who locate in different places to collaborate this proposing process by developing a web GIS application. Based on this success, some capabilities could be added to this application in the future.

In order to balance the protection level among the MBRs, the project was limited to considering the percentage of each MBR protected in MPAs. Other methods could be utilized to quantify the equality of protection, such as the Gini coefficient (Gini, 1921). Additionally, the protection among the marine biogeographic provinces and realms, which are larger units than MBRs, needs to be balanced. Besides the protection balance, some other factors could be considered when people propose a new MPA, such as animal behaviors and relevant policy. The application can be expanded to have further functionalities to implement these methods or consider other related factors.

A search function for users to help them explore the MPAs and MBRs efficiently could be added to the application. To locate an MPA or an MBR, users could search for it 
by name or the place around it. The application could have import/export data functions to let users upload or download the certain format files. The data format could be csv, shapefile, feature class and other common geographic data formats. In order to locate an MPA's position exactly, the application could provide a function to input the latitudes and longitudes for the vertexes of the MPA's polygon. It will be helpful if the users want to get the precise area and location for the MPA. 


\section{Works Cited}

ArcGIS TeamPython. (2013, May 20). Pairwise Intersect Tool. Retrieved March 20, 2016, from ArcGIS online:

http://www.arcgis.com/home/item.html?id=120807ac7f8449f89840ae48e87d7f55

Aswani, S., \& Lauer, M. (2006). Incorporating fishermen's local knowledge and behavior into geographical information systems (GIS) for designing marine protected areas in Oceania. Human Organization, 65(1), 81-102.

Barr, L. M., Pressey, R. L., Fuller, R. A., Segan, D. B., McDonald-Madden, E., \& Possingham, H. P. (2011). A new way to measure the world's protected area coverage. PLoS One, 6(9), e24707.

Barragan-Paladines, M. J., Chuenpagdee, R., Devillers, R., Edinger, E., Kerezi, V., \& Morgan, L. (2015). The Future of Marine Conservation: Local and Global Perspectives Workshop. Retrieved March 10, 2016, from https://globaloceanrefuge.org/wpcontent/uploads/2013/10/MPAs_Workshop_Report_Final.pdf

Briggs, J. C. (1974). Marine zoogeography. New York: McGraw-Hill.

Briggs, J. C. (1995). Global Biogeography. Amsterdam: Elsevier.

Ebach, M. C., \& Goujet, D. F. (2006). The first biogeographical map. Journal of Biogeography, 33(5), 761-769.

Esri. (2016). Calculate Field examples. Retrieved March 10, 2016, from ArcGIS for Desktop: http://desktop.arcgis.com/en/arcmap/10.3/manage-data/tables/calculatefield-examples.htm

Fernandes, L., Day, J. O. N., Lewis, A., Slegers, S., Kerrigan, B., Breen, D. A. N., . . Innes, J. (2005). Establishing representative no-take areas in the Great Barrier Reef: large-scale implementation of theory on marine protected areas. Conservation Biology, 19(6), 1733-1744.

Friedlander, A. M., Brown, E. K., \& Monaco, M. E. (2007). Coupling ecology and GIS to evaluate efficacy of marine protected areas in Hawaii. Ecological Applications, 17(3), 715-730.

Fu, P., \& Sun, J. (2010). Web GIS: principles and applications. Esri Press.

Gini, C. (1921). Measurement of inequality of incomes. The Economic Journal, 31(121), 124-126.

Halpern, B. S., \& Warner, R. R. (2002). Marine reserves have rapid and lasting effects. Ecology letters, 5(3), 361-366.

Harris, P. T., \& Whiteway, T. (2009). High seas marine protected areas: benthic environmental conservation priorities from a GIS analysis of global ocean biophysical data. Ocean \& Coastal Management, 52(1), 22-38.

Kairo, J. G., Kivyatu, B., \& Koedam, N. (2002). Application of remote sensing and GIS in the management of mangrove forests within and adjacent to Kiunga Marine Protected Area, Lamu, Kenya. Environment, Development and Sustainability, 4(2), 153-166.

Kendall, M. S., Christensen, J. D., \& Hillis-Starr, Z. (2003). Multi-scale data used to analyze the spatial distribution of French grunts, Haemulon flavolineatum, 
relative to hard and soft bottom in a benthic landscape. Environmental Biology of Fishes, 66(1), 19-26.

Longhurst, A. R. (1998). Ecological geography of the sea. San Diego: Academic Press.

Marine Conservation Institute. (2015). Sea States 2015 "How Well Does Your State Protect Your Coastal Waters?". Marine Conservation Institute.

Marine Conservation Institute. (2016). MPAtlas » Explore. Retrieved August 05, 2016, from MPAtlas: http://www.mpatlas.org/explore/

Olson, D. M., \& Dinerstein, E. (2002). The Global 200: Priority ecoregions for global conservation. Annals of the Missouri Botanical garden, 89(2), 199-224.

Roberts, C. M., Branch, G., Bustamante, R. H., Castilla, J. C., Dugan, J., Halpern, B. S., ... Warner, R. R. (2003). Application of ecological criteria in selecting marine reserves and developing reserve networks. Ecological applications, 13(1), S215S228.

Sherman, K., \& Alexander, L. M. (1989). Biomass yields and geography of large marine ecosystems. Boulder (CO): Westview Press.

Sherman, K., Sissenwine, M., Christensen, V., Duda, A., Hempel, G., Ibe, C., . . . Zwanenburg, K. (2005). A global movement toward an ecosystem approach to management of marine resources. Marine Ecology Progress Series 300, 275-279.

Spalding, M. D., Fox, H. E., Allen, G. R., Davidson, N., Ferdaña, Z. A., Finlayson, M. A., . . Martin, K. D. (2007). Marine ecoregions of the world: a bioregionalization of coastal and shelf areas. BioScience, 57(7), 573-583.

Wood, L. J., \& Dragicevic, S. (2007). GIS-based multicriteria evaluation and fuzzy sets to identify priority sites for marine protection. Biodiversity and Conservation, 16(9), 2539-2558.

Zharikov, Y., Skilleter, G. A., Loneragan, N. R., Taranto, T., \& Cameron, B. E. (2005). Mapping and characterising subtropical estuarine landscapes using aerial photography and GIS for potential application in wildlife conservation and management. Biological Conservation, 125(1), 87-100. 


\section{Appendix A. HTML Code}

HTML Code for Application

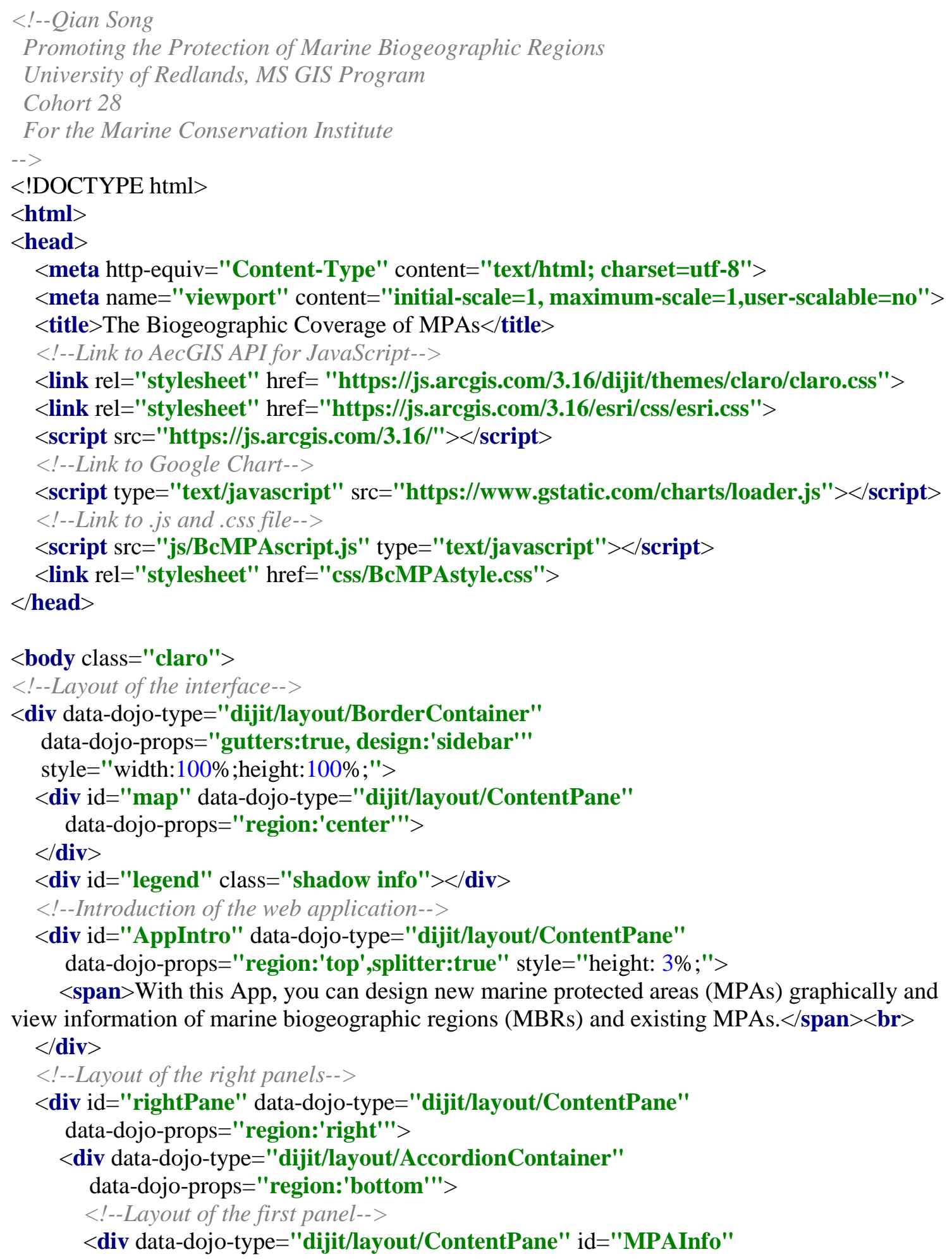




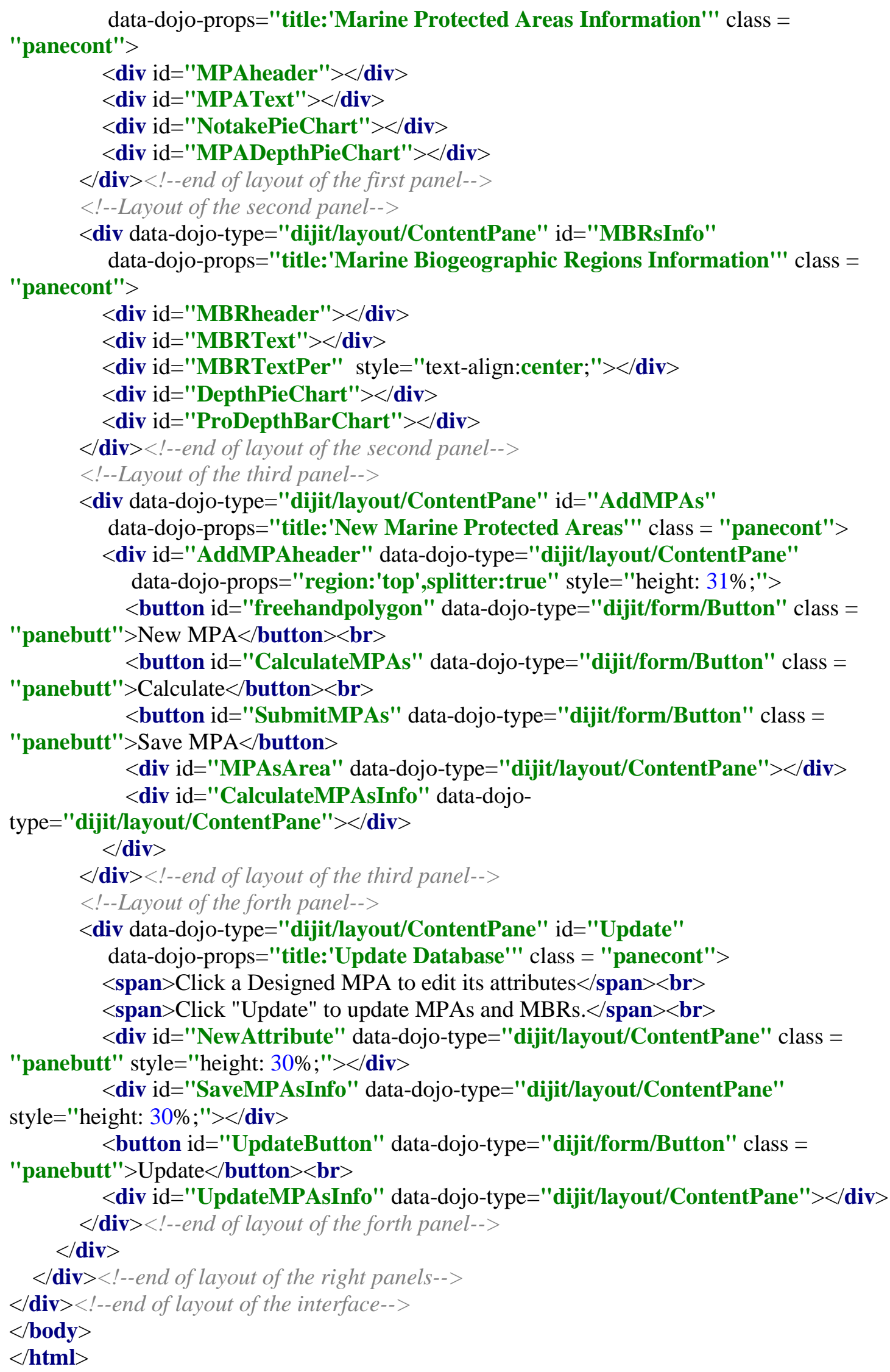




\section{Appendix B. JavaScript Code}

JavaScript Code for the Application

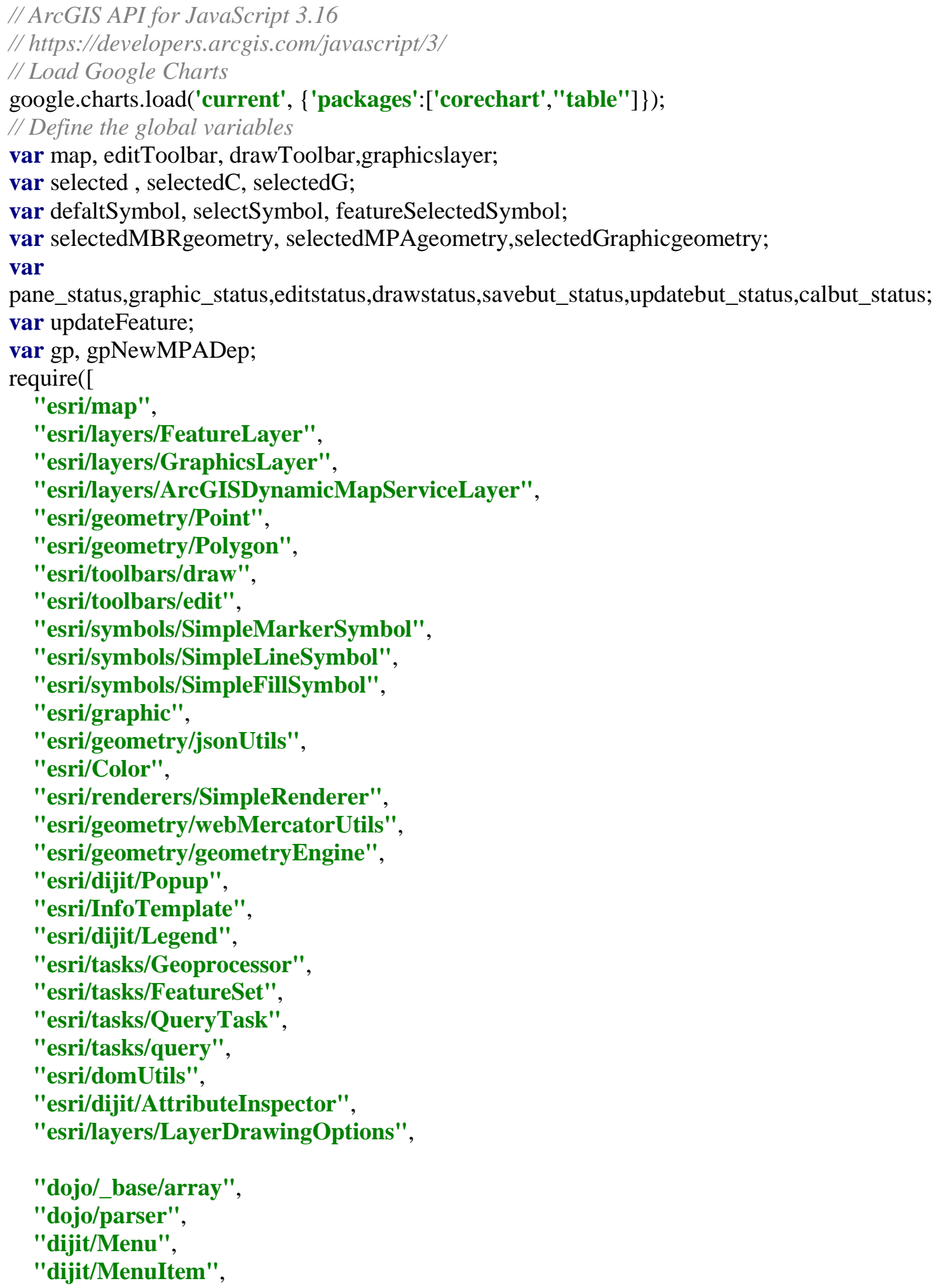




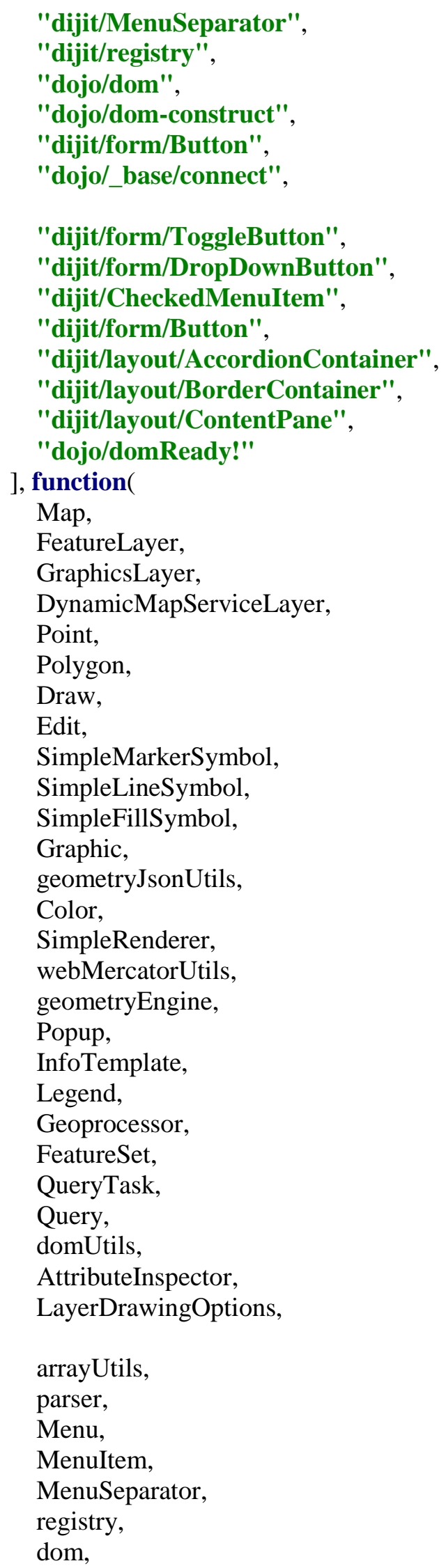


domConstruct,

Button,

connect

) \{

parser.parse();

IIIIIIIII Set symbol for features

defaltSymbol = new SimpleFillSymbol().setColor(new Color $([0,200,0,0.5]))$;

selectSymbol= new SimpleFillSymbol().setColor $($ new Color $([200,0,0,0.5]))$;

featureSelectedSymbol = new SimpleFillSymbol().setColor $($ new Color $([0,0,200,1]))$;

IIIIIIII/ Setup popup

var popup = new esri.dijit.Popup $(\{$ fillSymbol:featureSelectedSymbol $\}$, dojo.create("div"));

var ext $=$ new esri.geometry.Extent $(\{$

"xmin": -180 ,

"ymin": -60 ,

"xmax": 180,

"ymax": 60,

\}$)$;

// Define and configure a new map

map = new Map("map", \{

basemap: "oceans",

center: $[-60,5]$,

zoom: 4 ,

minScale: 100000000 ,

"extent": ext,

\}$)$;

"infoWindow": popup,

IIIIIIIII Marine Biogeographic Regions (MBR) Feature Service

var MBR_FC = new FeatureLayer("https://msgis-webdev-

1.redlands.edu/arcgis/rest/services/qian_song/BR_MS/FeatureServer/0", ,

mode: FeatureLayer.MODE_AUTO,

outFields:

["ECO_CODE",,"ProDep0_200","ProDep200_1000","ProDepAbove1000",,"geoArea","EC

OREGION",,"PROVINCE",,"REALM","Dep0_200","Dep200_1000",,"DepAbove1000"], \}$)$;

IIIIIII/I Marine Protected Areas (MPA) Feature Service

var MPA_FC = new FeatureLayer("https://msgis-webdev-

1.redlands.edu/arcgis/rest/services/qian_song/MPA_MS/FeatureServer/0", \{ mode: FeatureLayer.MODE_AUTO, outFields:

["name","geoArea","'no_take_sum","Dep0_200","Dep200_1000",,"DepAbove1000"], \}$)$

I/IIIIII/ Marine Biogeographic Regions Map Service

var MBR_MC = new DynamicMapServiceLayer("https://msgis-webdev-

1.redlands.edu/arcgis/rest/services/qian_song/BR_MS/MapServer", \{

opacity: 0.8 ,

\}$)$

I/IIIIIII Marine Protected Areas Map Service 
var MPA_MC = new DynamicMapServiceLayer("'https://msgis-webdev1.redlands.edu/arcgis/rest/services/qian_song/MPA_MS/MapServer", \{

opacity: 0.8 ,

\}$)$;

I/IIIIIII Designed Marine Protected Areas Feature Serivce

var NewMPA_FC = new FeatureLayer("https://msgis-webdev-

1.redlands.edu/arcgis/rest/services/qian_song/MIP_services77/FeatureServer/0", \{ mode: FeatureLayer.MODE_AUTO, outFields: ["*"]

\}$)$

I/IIIIII Graphic Layer for designed Marine Protected Areas

graphicslayer $=$ new GraphicsLayer();

I/IIIIII Define a layerlist and push layers to the list

var layerlist $=[]$;

layerlist.push(MBR_FC);

layerlist.push(MPA_FC);

layerlist.push(MBR_MC);

layerlist.push(MPA_MC);

layerlist.push(NewMPA_FC);

layerlist.push(graphicslayer);

IIIIIIII Set symbol for MBR Map Service

var symbol2 = new SimpleFillSymbol().setColor(new Color([130,221,218,1]));

var layerDrawingOptions2 = [];

var layerDrawingOption2 = new LayerDrawingOptions();

layerDrawingOption2.renderer $=$ new SimpleRenderer(symbol2);

layerDrawingOptions2[0] = layerDrawingOption2;

MBR_MC.setLayerDrawingOptions(layerDrawingOptions2);

I/IIIIII Set symbol for MPA Map Service

var symbol3 = new SimpleFillSymbol().setColor(new Color([68,106,142,1]));

var layerDrawingOptions3 = [];

var layerDrawingOption3 = new LayerDrawingOptions();

layerDrawingOption3.renderer $=$ new SimpleRenderer(symbol3);

layerDrawingOptions3[0] = layerDrawingOption3;

MPA_MC.setLayerDrawingOptions(layerDrawingOptions3);

IIIIIIII Set symbol for Designed Marine Protected Areas

var symbol4 = new SimpleFillSymbol().setColor(new Color([255,255,20,0.8]));

var renderer4 = new SimpleRenderer(symbol4);

layerlist[4].setRenderer(renderer4);

I/IIIIII/ Set symbol for graphicslayer

var symbol5 = new SimpleFillSymbol().setColor(new Color([0,200,0,0.8]));

var renderer5 = new SimpleRenderer(symbol5);

layerlist[5].setRenderer(renderer5);

map.addLayers([MBR_MC,MPA_MC,NewMPA_FC,graphicslayer]);

I/IIIII/I Initialize Application, call pane 1 function 


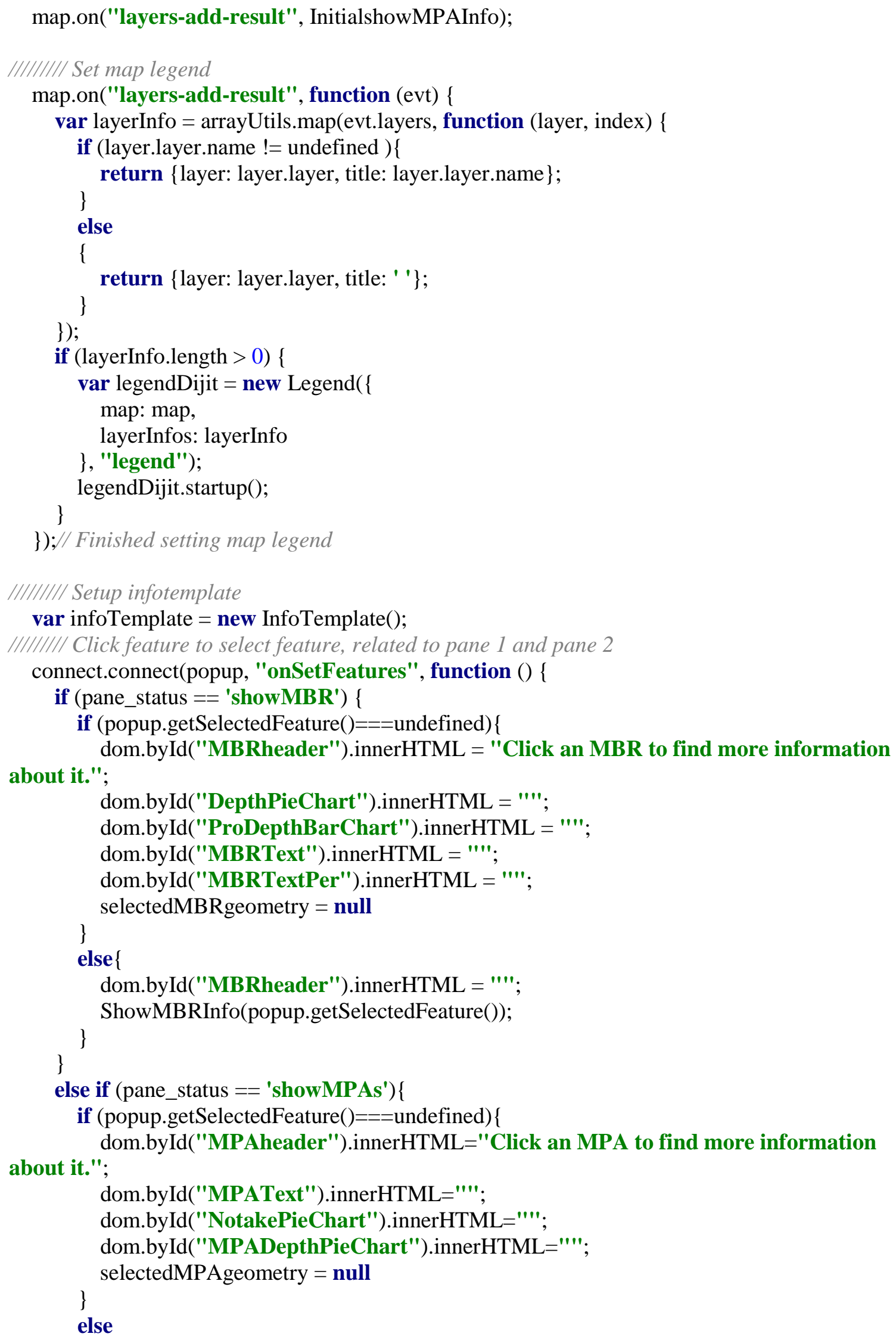




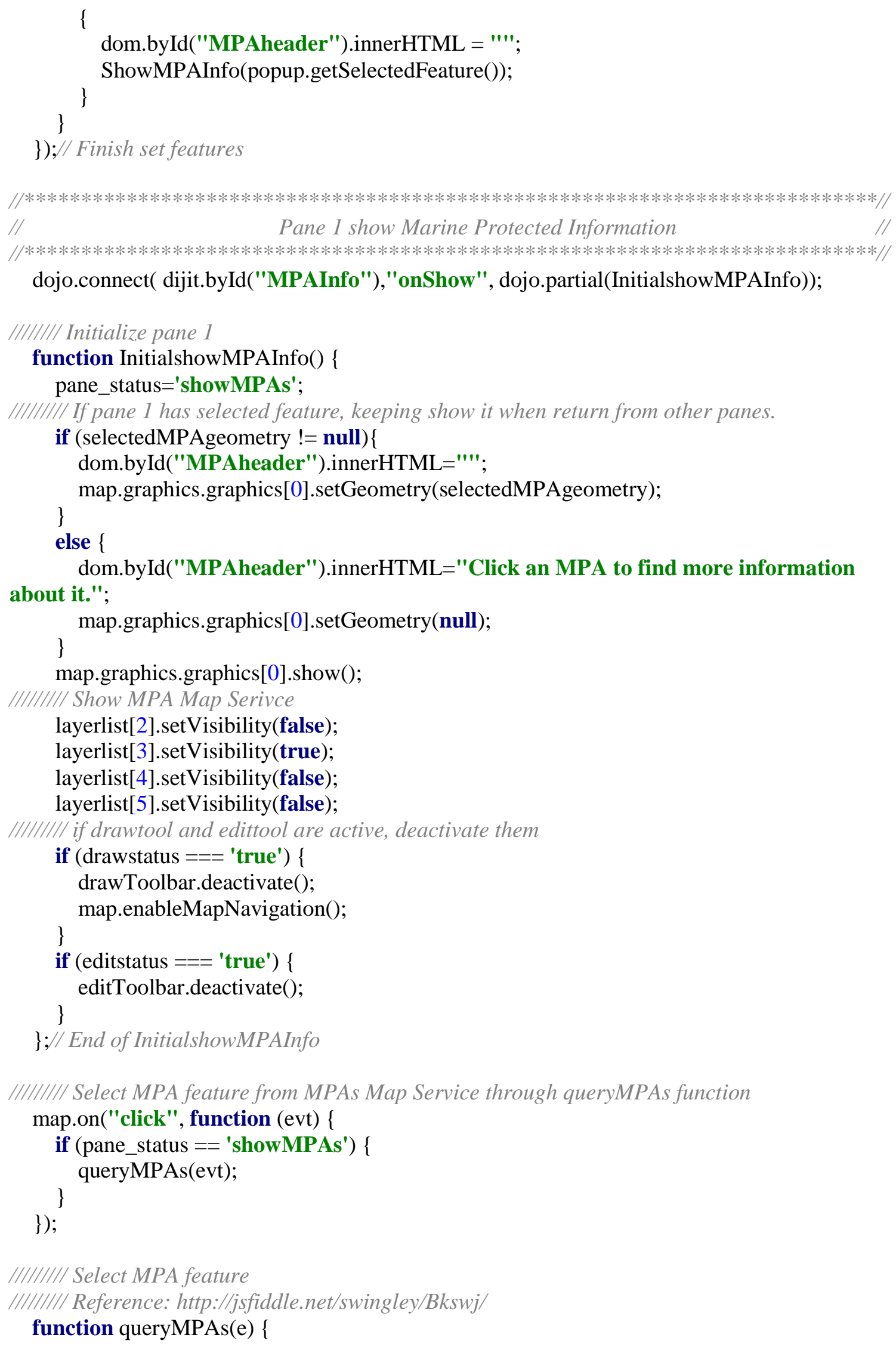

function queryMPAs(e) \{ 
I/IIIIII Build an extent around the click point

var pad = map.extent.getWidth() / map.width * 3;

var queryGeom $=$ new esri.geometry.Extent(e.mapPoint.x - pad, e.mapPoint.y - pad,

e.mapPoint.x + pad, e.mapPoint.y + pad, map.spatialReference);

var $q=$ new esri.tasks.Query();

q.returnGeometry = true;

q.outFields = ["name", "no_take_sum",

"geoArea","Dep0_200",,"Dep200_1000",,"DepAbove1000"];

q.geometry = queryGeom;

var popupTemplate $=$ new esri.dijit.PopupTemplate () ;

var qt = new esri.tasks.QueryTask("https://msgis-webdev-

1.redllands.edu/arcgis/rest/services/qian_song/MPA_MS/MapServer/0");

def = qt.execute $(q)$;

def.addCallback(function (result) \{

result.features.sort(function(a,b) \{return a.attributes.geoArea - b.attributes.geoArea $\}$ )

return dojo.map([result.features[0]], function (f) \{

f.setInfoTemplate(popupTemplate);

return $\mathrm{f}$;

\}$)$

\}$)$

IIIIIIIII Use the deferred returned from the query task to set the popup features

map.infoWindow.setFeatures([def]);

\}// End of queryMPAs

IIIIIII Show MPA information inside pane

function ShowMPAInfo(feature) \{

if (feature) \{

selectedMPAgeometry=feature.geometry;

dom.byId("MPAText").innerHTML =feature.attributes.name.bold().fontsize(5) + " $<$ br $><$ br $>$ ";

if $($ feature.attributes.geoArea $==$ null $)\{$

dom.byId("'DepthPieChart").innerHTML = 'Sorry, data are not completed!'; \}

else \{

createNotakePieChart(feature);

createMPADepthPieChart(feature);

\}

\} // Finish if (feature)

\} // End of ShowMBRInfo

IIIIIIII Show no take area information through pie chart

function createNotakePieChart(feature) \{

var Notake= feature.attributes.no_take_sum;

var Restric $=$ feature.attributes.geoArea - feature.attributes.no_take_sum;

var Restric_show $=0$

if (Notake>feature.attributes.geoArea) \{

Restric_show $=0$

\}

else \{

\}

Restric_show=Restric 


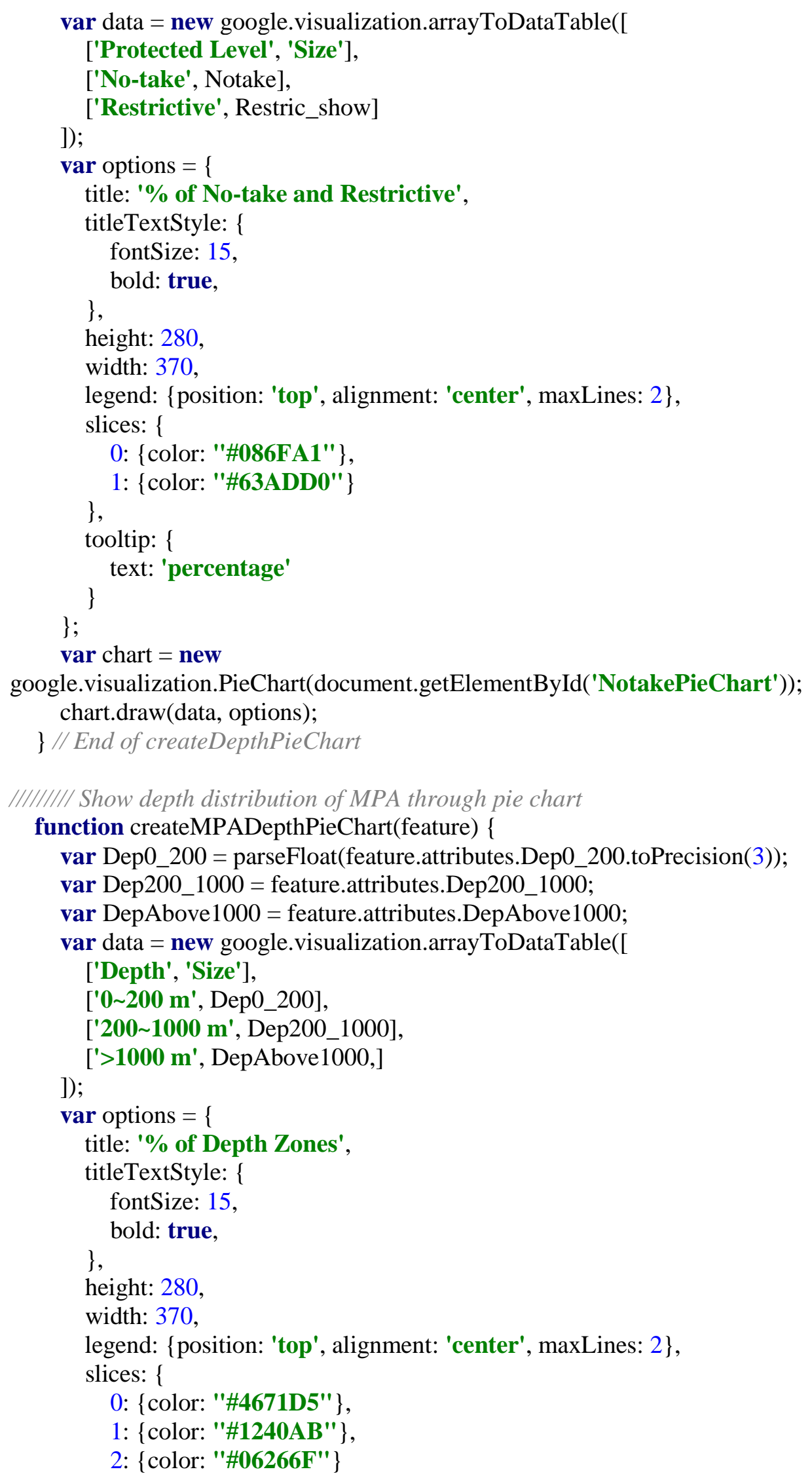




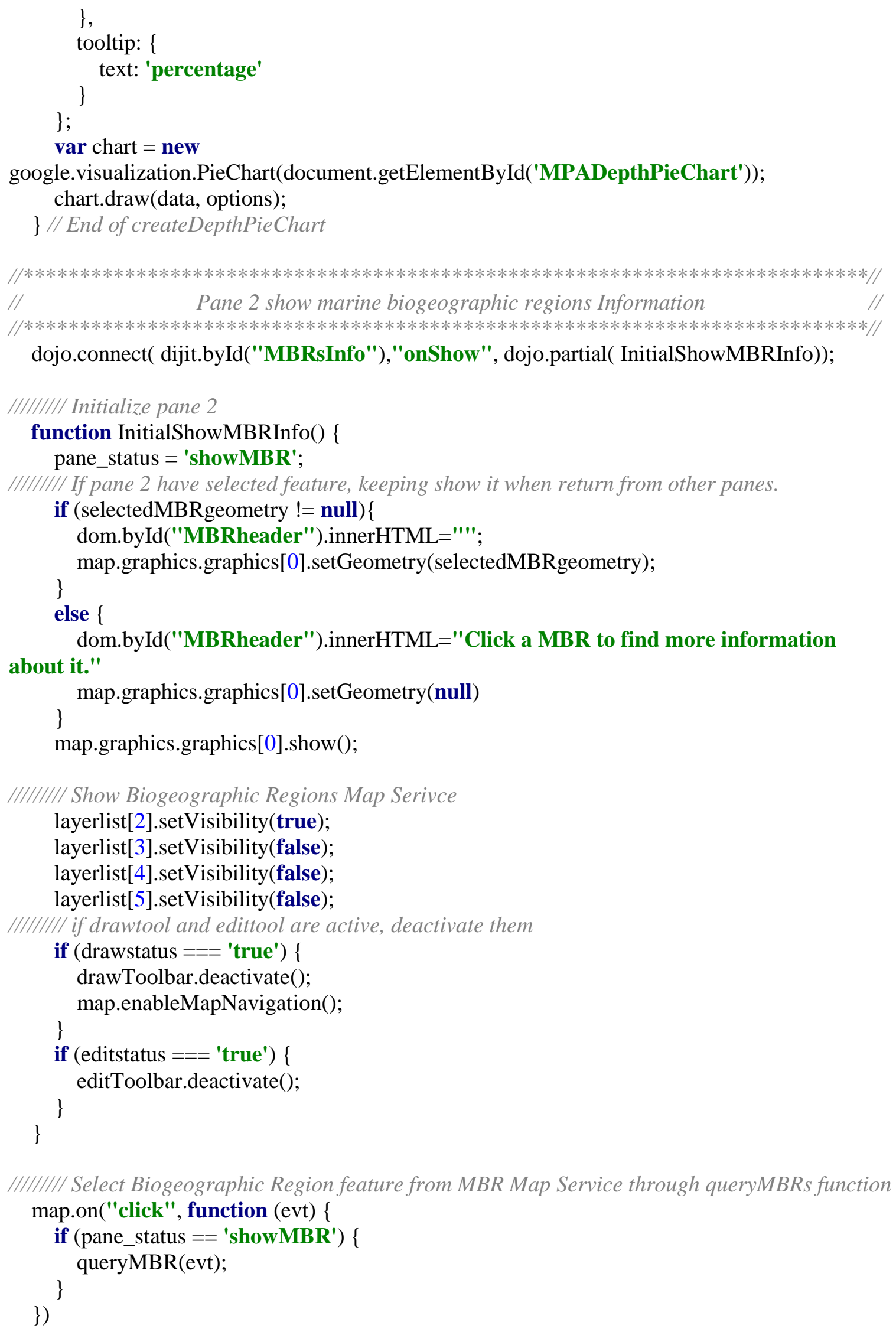




\section{I/IIIIII Select Biogeographic Region feature}

function queryMBR(e) \{

IIIIIIIII Build an extent around the click point

var pad = map.extent.getWidth() / map.width * 3;

var queryGeom = new esri.geometry.Extent(e.mapPoint.x - pad, e.mapPoint.y - pad,

e.mapPoint.x + pad, e.mapPoint.y + pad, map.spatialReference);

$\operatorname{var} \mathrm{q}=$ new esri.tasks.Query();

q.returnGeometry = true;

q.outFields $=$

["ECO_CODE","ProDep0_200",,"ProDep200_1000",,"ProDepAbove1000","geoArea","EC

OREGION","PROVINCE","REALM","Dep0_200","Dep200_1000","DepAbove1000"];

q.geometry = queryGeom;

var popupTemplate $=$ new esri.dijit.PopupTemplate () ;

var qt = new esri.tasks.QueryTask("https://msgis-webdev-

1.redlands.edu/arcgis/rest/services/qian_song/BR_MS/MapServer/0");

def $=$ qt.execute $(q)$;

def.addCallback(function (result) \{

result.features.sort(function $(\mathrm{a}, \mathrm{b})\{$ return a.attributes.geoArea - b.attributes.geoArea $\})$

return dojo.map([result.features[0]], function (f) \{

f.setInfoTemplate(popupTemplate);

return $\mathrm{f}$;

\}$)$

\}$)$

IIIIIIIII Use the deferred returned from the query task to set the popup features

map.infoWindow.setFeatures([def]);

\}; //End of queryMBR

IIIIIIII Show Biogeographic Region information inside pane

function ShowMBRInfo(feature) \{

if (feature) \{

selectedMBRgeometry=feature.geometry;

var

MBR_percent=(feature.attributes.ProDep0_200+feature.attributes.ProDep200_1000+feature.attri

butes.ProDepAbove1000)/feature.attributes.geoArea*100;

dom.byId("MBRText").innerHTML =feature.attributes.ECOREGION.bold().fontsize(5) + "<br $>"$

+"<br>Province: " + feature.attributes.PROVINCE +

"<br>Realm: " + feature.attributes.REALM +

"<br>Percentage of Protection:"

dom.byId("MBRTextPer").innerHTML =MBR_percent.toFixed(2).bold().fontsize(6) + "\%<br $><b r>"$;

createDepthPieChart(feature);

createProDepthBarChart(feature)

\} // Finish if (iffeature)

\} // End of ShowMBRInfo

IIIIIIII Show depth distribution of biogeographic region through pie chart

function createDepthPieChart(feature) \{

var Dep0_200 = parseFloat(feature.attributes.Dep0_200.toPrecision(3));

var Dep200_1000 = feature.attributes.Dep200_1000; 
var DepAbove1000 = feature.attributes.DepAbove1000;

var data $=$ new google.visualization. arrayToDataTable $([$

['Depth', 'Size'],

['0 200 m', Dep0_200],

['200 1000 m', Dep200_1000],

]);

['>1000 m', DepAbove1000,]

var options $=\{$

title: '\% of Depth Zones',

titleTextStyle: \{

fontSize: 15 ,

bold: true,

\},

height: 280 ,

width: 370 ,

legend: \{position: 'top', alignment: 'center', maxLines: 2 \},

slices: \{

0: $\{$ color: "\#4671D5" \},

1: $\{$ color: "\#1240AB" $\}$,

2: $\{$ color: "\#06266F" $\}$

\},

tooltip: \{

\}

text: 'percentage'

\} ;

var chart $=$ new

google.visualization.PieChart(document.getElementById('DepthPieChart')); chart.draw(data, options);

\} // End of createDepthPieChart

IIIIIIIII Show percentages of different depth regions protected in MPAs

function createProDepthBarChart(feature) \{

var Dep0_200 = feature.attributes.Dep0_200;

var Dep200_1000 = feature.attributes.Dep200_1000;

var DepAbove 1000 = feature.attributes.DepAbove1000;

var ProDep0_200 = feature.attributes.ProDep0_200;

var ProDep200_1000 = feature.attributes.ProDep200_1000;

var ProDepAbove 1000 = feature.attributes.ProDepAbove1000;

var PerDep_0_200 = ProDep0_200/Dep0_200;

var PerDep_200_1000 = ProDep200_1000/Dep200_1000;

var PerDep_above1000 = ProDepAbove1000/DepAbove 1000;

var PerDep = new google.visualization. arrayToDataTable $([$

['Depth', 'Protected', 'Unprotected'],

['0 200 m', PerDep_0_200, (1 - PerDep_0_200)],

['200 1000 m', PerDep_200_1000, (1 - PerDep_200_1000)],

['>1000 m', PerDep_above1000, (1 - PerDep_above1000)]

]);

var formatter $=$ new google.visualization.NumberFormat $(\{$

pattern: '\#\%',

fractionDigits: 2

\}$)$ 


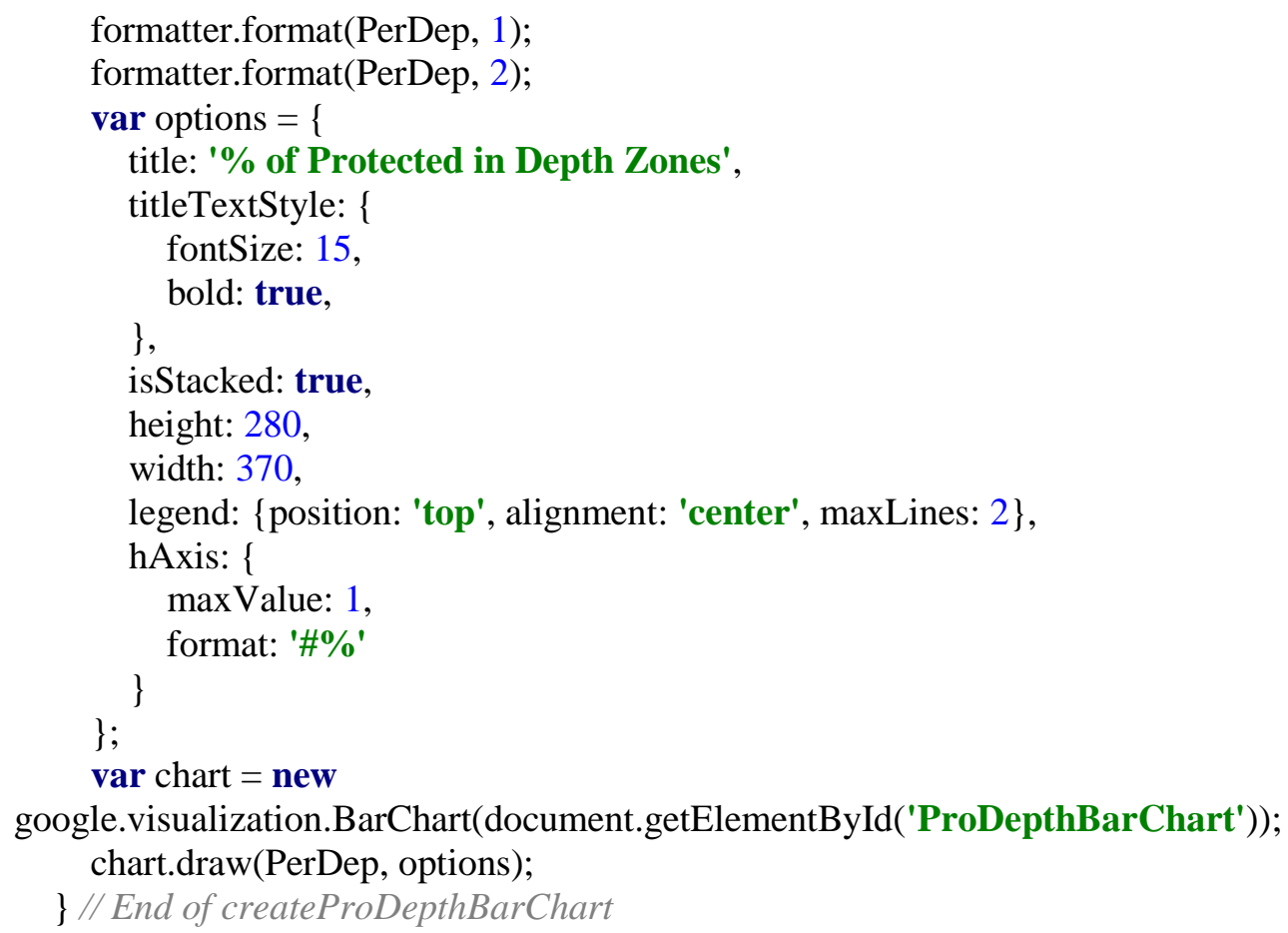




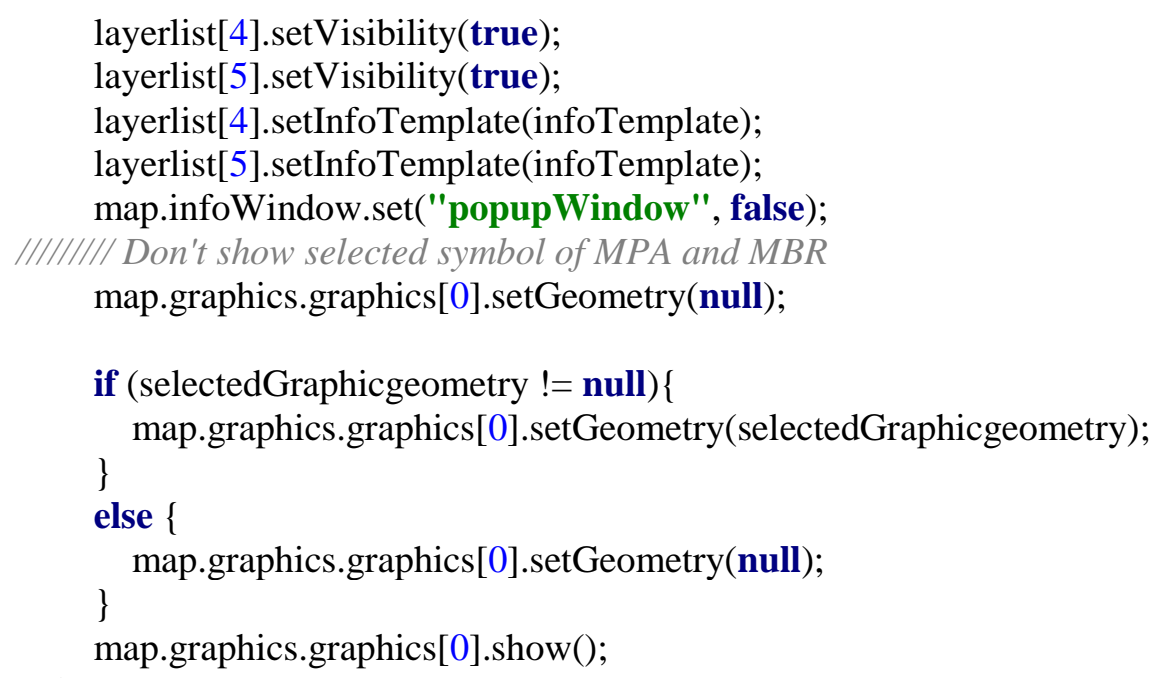


map.graphics.graphics[0].setGeometry(null);

I/IIIII/ Finish edit a graphic in graphic layer, set it as defaltSymbol

if (evt.graphic.attributes $===$ undefined) \{

evt.graphic.setSymbol(defaltSymbol);

selectedC = undefined;

\}

if (evt.graphic.attributes != undefined) \{

IIIIIIII Finish edit a feature in Designed feature layer, save the feature and clear selected feature

layerlist[4].applyEdits(null, [evt.graphic], null);

layerlist[4].clearSelection();

selectedC = undefined;

registry.byId("CalculateMPAs").setDisabled(true);

\}

evt.graphic.setSymbol(layerlist[4].renderer.symbol)

\}$)$;

editToolbar.on("activate", function(evt) \{

editstatus = 'true';

map.graphics.graphics[0].setGeometry(null);

dom.byId("CalculateMPAsInfo").innerHTML = "Calculate the percentages of

protected coverages in related MBRs.";

dom.byId("MPAsArea").innerHTML="After editing, please click a New MPA or a

designed MPA to calculate the area and the perimeter.";

evt.graphic.setSymbol(selectSymbol);

if (evt.graphic.attributes != undefined) \{

evt.graphic.attributes["status"] = "area_changed";

dom.byId('SaveMPAsInfo').innerHTML = "MPA has been edited, please save

attributes again";

\}

\});// End of editToolbar status

drawGraphic();

editGraphic();

\}// End of InitToolbar

I/IIIIII/ Actions of "New MPA" button

registry.byId("freehandpolygon").on("click", function() \{

if (drawstatus === 'true') \{

drawToolbar.deactivate();

map.enableMapNavigation();

\}

else if (drawstatus === 'false') \{

map.disableMapNavigation();

editToolbar.deactivate();

drawToolbar.activate(this.id);

\}

else \{

map.disableMapNavigation();

editToolbar.deactivate();

drawToolbar.activate(this.id);

\}

\});//End of "New MPA" button 


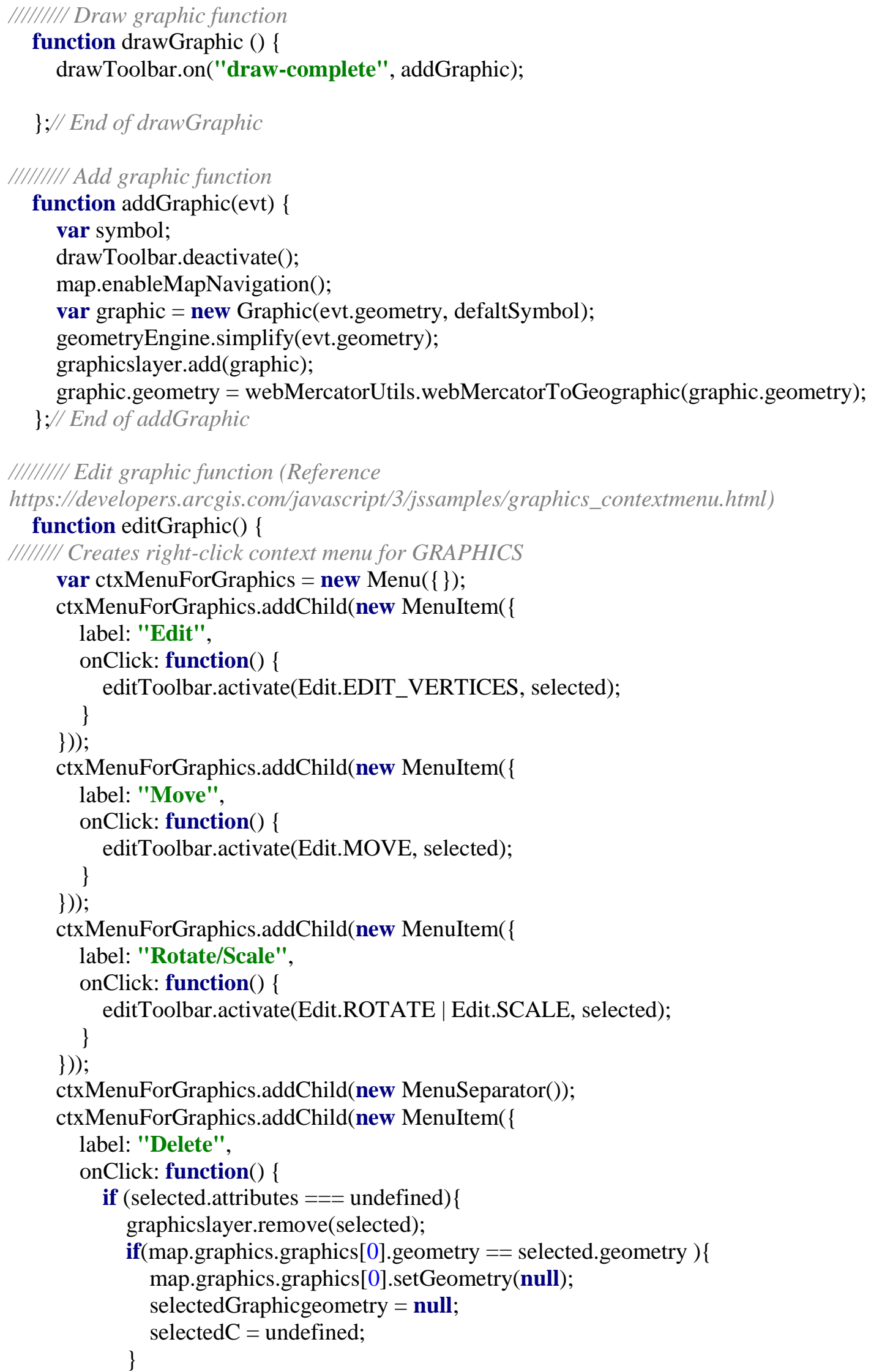




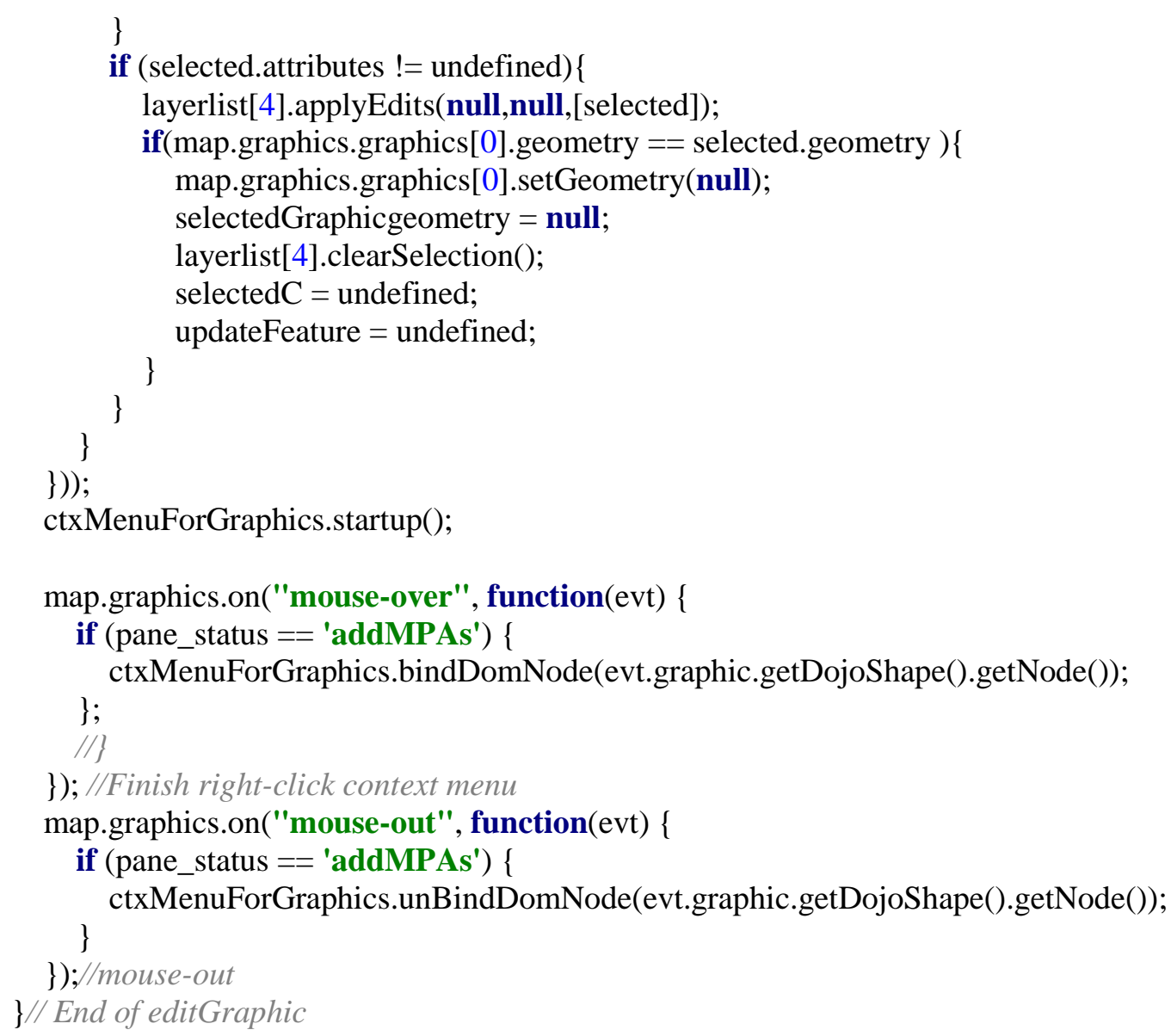

IIIIIIII Click a graphic to calculate its area and perimeter

dojo.connect(layerlist[5],"onClick", function (evt) \{

IIIIIIIII If Calculate is not active, MPA graphic layer can be selected.

if (pane_status === 'addMPAs' $\& \&$ calbut_status == 'deactivate') \{ graphic_status = 'graphicsL';

layerlist[4].clearSelection();

///selectedC = evt.graphic;

selectedG = evt.graphic;

selected $=$ evt.graphic;

selectedGraphicgeometry = evt.graphic.geometry;

calculateGraphic(evt);

dom.byId("CalculateMPAsInfo").innerHTML = "Click 'Calculate' to calculate the

percentages of protected coverages in related MBRs."; registry.byId("CalculateMPAs").setDisabled(false);

registry.byId("SubmitMPAs").setDisabled(false);

\}

\});// End of click a graphic to calculate its area and perimeter

IIIIIIII Click a feature to calculate its area and perimeter

dojo.connect(layerlist[4],"onClick",function (evt)\{

IIIIIIIII If Calculate is not active, Designed MPA features can be selected.

if (pane_status == 'addMPAs' \& \& calbut_status == 'deactivate') \{ graphic_status = 'featureL';

var query = new Query(); 
query.geometry = evt.graphic.geometry;

layerlist[4].selectFeatures(query, layerlist[4].SELECTION_NEW, function (features) \{ selected $=$ features $[0]$;

updateFeature $=$ features $[0]$;

\}$)$

selectedGraphicgeometry = evt.graphic.geometry;

calculateGraphic(evt);

dom.byId("CalculateMPAsInfo").innerHTML = "Click 'Calculate' to calculate the

percentages of protected coverages in related MBRs.";

registry.byId("CalculateMPAs").setDisabled(false);

registry.byId("SubmitMPAs").setDisabled(true);

\}

\});// End of click a feature to calculate its area and perimeter

I/IIIIII/ Calculate area and perimeter of a selected graphic or feature

function calculateGraphic(evt) \{

selectedC=evt.graphic;

//selectedC.setSymbol(selectSymbol);

var temp_area = geometryEngine.geodesicArea(evt.graphic.geometry, "square-

kilometers" );

var temp_length = geometryEngine.geodesicLength(evt.graphic.geometry, "kilometers" ); dom.byId("MPAsArea").innerHTML="Area: "+ temp_area.toFixed(0) + " square kilometers"+

"<br>"+"Perimeter: "+ temp_length.toFixed $(0)+$ " kilometers";

\};/ End of calculateGraphic

IIIIIIII Calculate pertcentage of related MBRs protected in a designed MPA.

gp = new Geoprocessor("https://msgis-webdev-

1.redlands.edu/arcgis/rest/services/qian_song/CalProtectedMEOW/GPServer/Script" )

I/IIIIII Actions of "Calculate" button

registry.byId("CalculateMPAs").on("click", function() \{

calbut_status = 'activate';

layerlist[4].setInfoTemplate(null);

layerlist[5].setInfoTemplate(null);

var features=[];

features.push(selectedC);

var featureSet= new FeatureSet();

featureSet.features=features;

var params $=\{$ "InputNewMPA":featureSet $\}$;

if (featureSet.features[0] == undefined) \{

dom.byId("CalculateMPAsInfo").innerHTML ="Please select an area.";

\}

else if (editstatus $===$ 'true') \{

dom.byId("CalculateMPAsInfo").innerHTML ="Please finish editing.";

\}

else \{

gp.submitJob(params, completeCallback, statusCallback);

\}

\}$)$ 


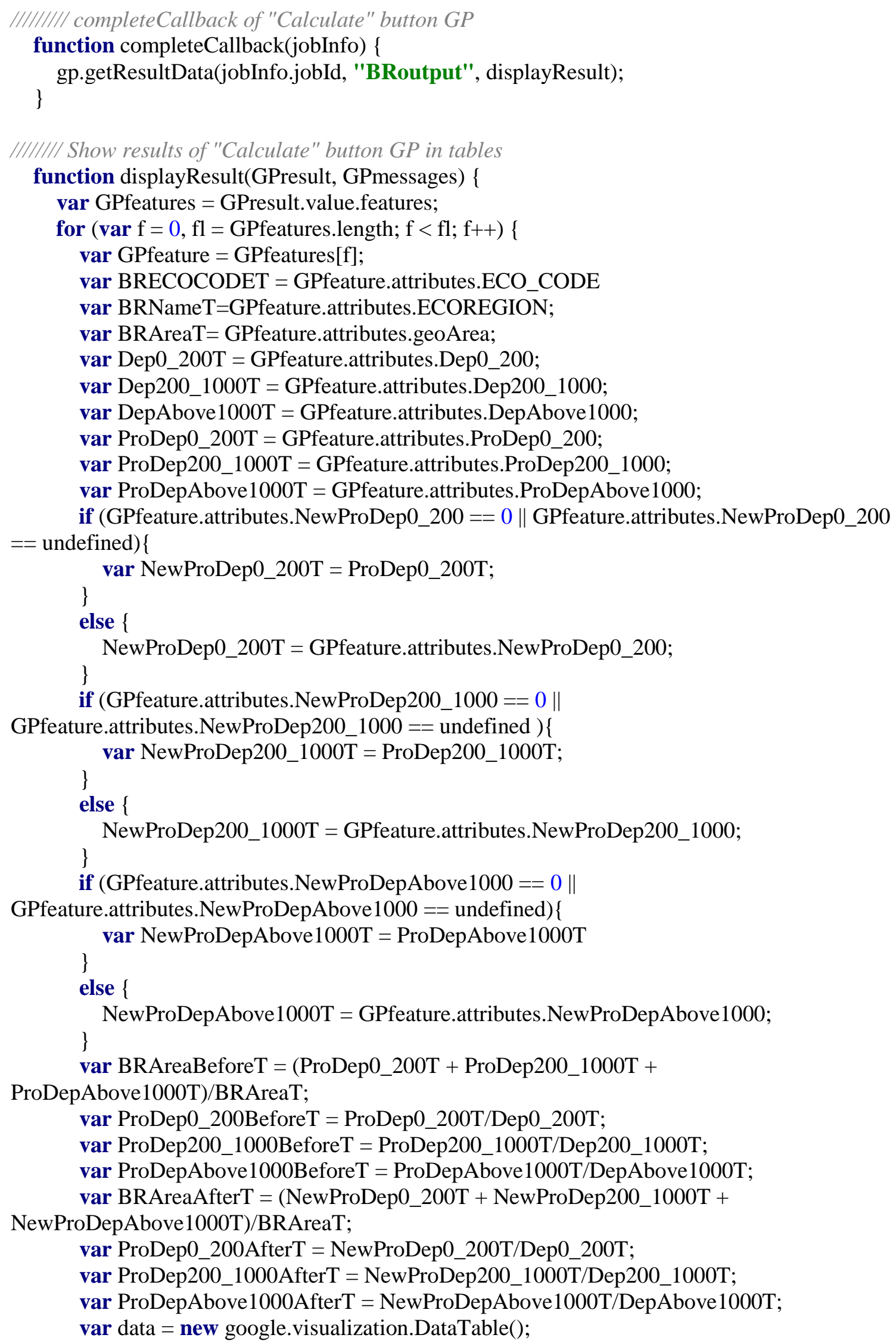




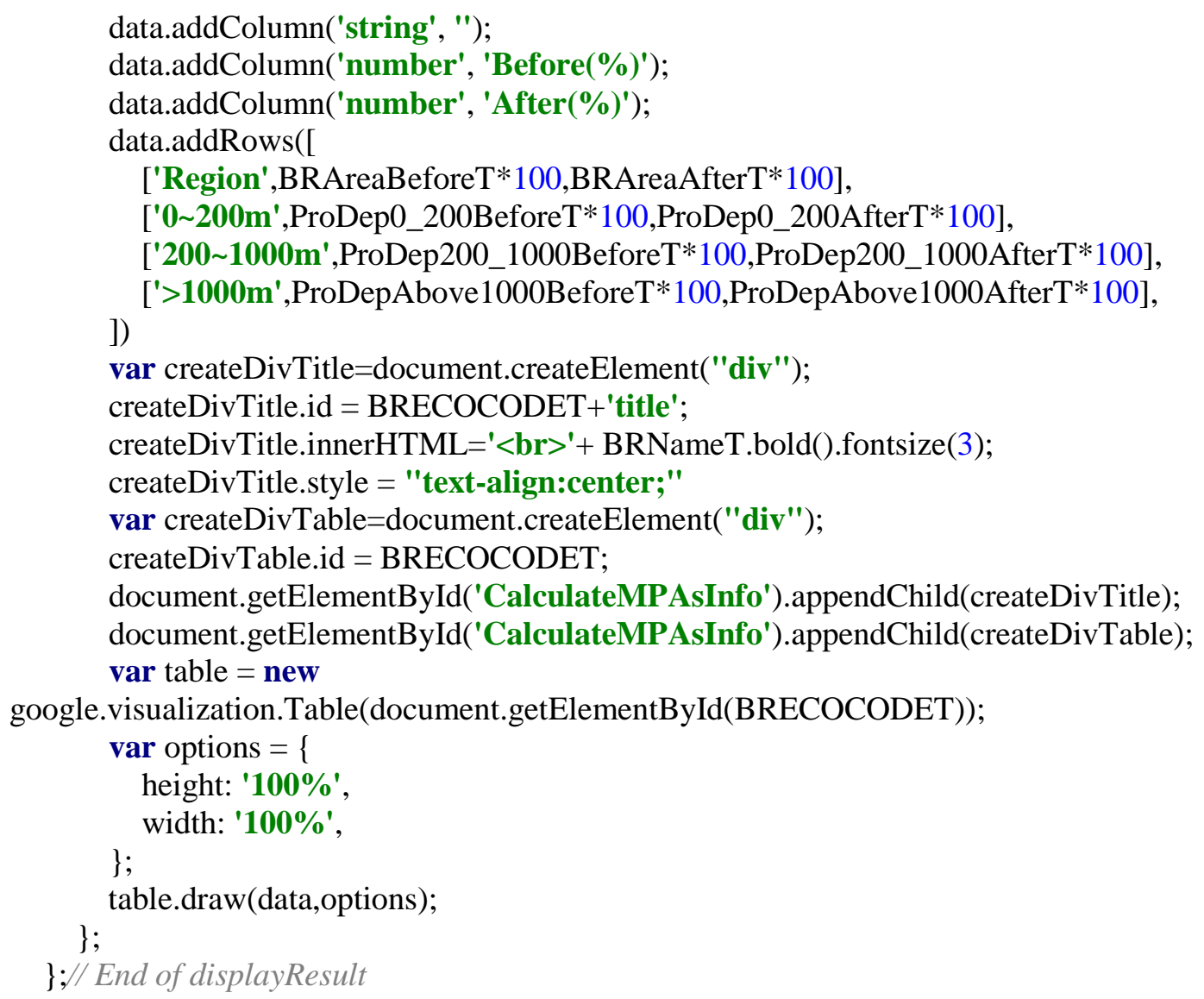




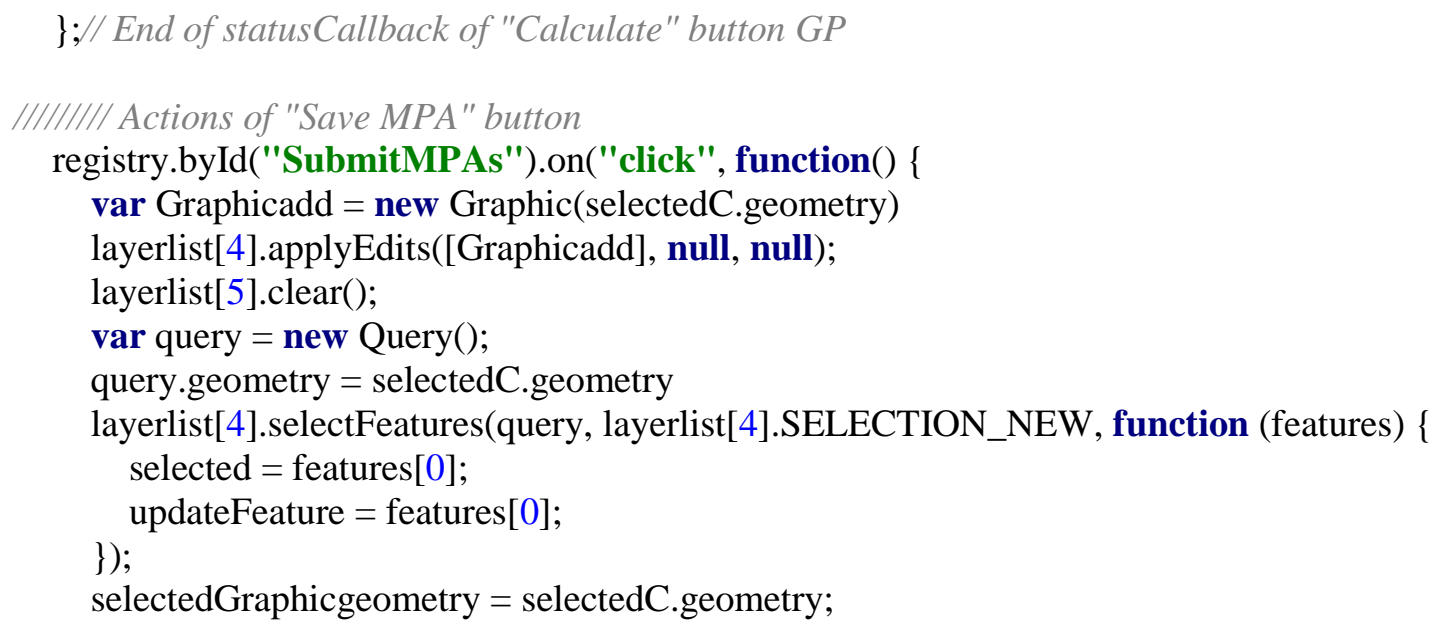


\}

else \{

map.graphics.graphics[0].setGeometry(null);

\}

map.graphics.graphics[0].show();

var updateFeaturelist = layerlist[4].getSelectedFeatures();

updateFeature $=$ updateFeaturelist $[0]$;

IIIIIIIIII If the selected MPA is edited, its attributes are changed, click Save again

if (updateFeature.attributes["status"] === "area_changed") \{

dom.byId("SaveMPAsInfo").innerHTML="The MPA has been edited, please save attributes again ";

\}

else \{

dom.byId("SaveMPAsInfo").innerHTML = " ";

IIIIIIIII If Designed MPA feature doesn't have key attributes, it cannot be updated

if (updateFeature.attributes["name"] != null \&\&

updateFeature.attributes["name"] != "' \&\& updateFeature.attributes["name"] != undefined) \{

if (updateFeature.attributes["mpa_id"] != null \&\&

is $\mathrm{NaN}$ (updateFeature.attributes["mpa_id"]) == false \&\& updateFeature.attributes["mpa_id"] != undefined) \{

if (updateFeature.attributes["Dep0_200"] != undefined \&\&

updateFeature.attributes["Dep200_1000"] != undefined \&\&

updateFeature.attributes["DepAbove1000"] != undefined) \{

registry.byId("UpdateButton").setDisabled(false);

\}

\}

\}

\};// End of (If a Designed MPA is selected, enable "Update" button)

\};// End of

map.on("click", function () \{

if (pane_status == 'update' $\& \&$ savebut_status == 'deactivate' $\& \&$ updatebut_status == 'deactivate')\{

if (popup.getSelectedFeature ()$===$ undefined $)\{$

dom.byId("CalculateMPAsInfo").innerHTML = "Click 'Calculate' to calculate the

percentages of protected coverages in related MBRs.";

dom.byId("MPAsArea").innerHTML="Please click a New MPA or a designed MPA

to calculate the area and the perimeter.";

dom.byId("UpdateMPAsInfo").innerHTML=" ";

dom.byId("SaveMPAsInfo").innerHTML=" ";

selectedC = undefined;

updateFeature $=$ undefined;

selectedGraphicgeometry = null;

layerlist[4].clearSelection();

registry.byId("UpdateButton").setDisabled(true);

registry.byId("CalculateMPAs").setDisabled(true);

registry.byId("SubmitMPAs").setDisabled(true);

\}

\} ); 
IIIIIIII Select a feature of Designed MPA

dojo.connect(layerlist[4],"onClick",function (evt)\{

registry.byId("UpdateButton").setDisabled(true);

IIIIIIIII If the Save and Update are not active, Designed MPA features can be selected.

if (pane_status $==$ 'update' $\& \&$ savebut_status $==$ 'deactivate' $\& \&$ updatebut_status $==$ 'deactivate') \{

var query = new Query();

query.geometry = evt.graphic.geometry;

layerlist[4].selectFeatures(query,layerlist[4].SELECTION_NEW,function (features) \{

updateFeature $=$ features[0];

selected $=$ features $[0]$;

console.log('showupdateF',updateFeature.attributes["mpa_id"])

IIIIIIIII If the selected MPA is edited, its attributes are changed, click Save again

if (updateFeature.attributes["status"] === "area_changed") \{

dom.byId("SaveMPAsInfo").innerHTML="The MPA has been edited, please

save attributes again ";

\}

else \{

dom.byId("SaveMPAsInfo").innerHTML = " ";

IIIIIIIII If Designed MPA feature doesn't have key attributes, it cannot be updated

if (updateFeature.attributes["name"] != null \&\&

updateFeature.attributes["name"] != "" \&\& updateFeature.attributes["name"] != undefined) \{

if (updateFeature.attributes["mpa_id"] != null \&\&

isNaN(updateFeature.attributes["mpa_id"]) == false \&\& updateFeature.attributes["mpa_id"] != undefined) \{

if (updateFeature.attributes["Dep0_200"] != undefined \&\&

updateFeature.attributes["Dep200_1000"] != undefined \&\&

updateFeature.attributes["DepAbove1000"] != undefined) \{ registry.byId("UpdateButton").setDisabled(false);

\}

\}

\}

\}

\}$)$

selectedGraphicgeometry = evt.graphic.geometry;

calculateGraphic(evt);

dom.byId("CalculateMPAsInfo").innerHTML = "Click 'Calculate' to calculate the

percentages of protected coverages in related MBRs.";

\}; //InitialUpdate

\});// End of (Select a feature of Designed MPA)

IIIIIIII Initialize Attribute Inspector (Reference

http://gis23.fortlauderdale.gov/jsapi/sdk312/jssamples/ed_attribute_inspector.html)

var layerInfos $=[$

\{

'featureLayer': layerlist[4],

'showAttachments': false,

'isEditable': true,

'fieldInfos': [

\{'fieldName': 'name', 'isEditable': true, 'label': '* Name:'\}, 


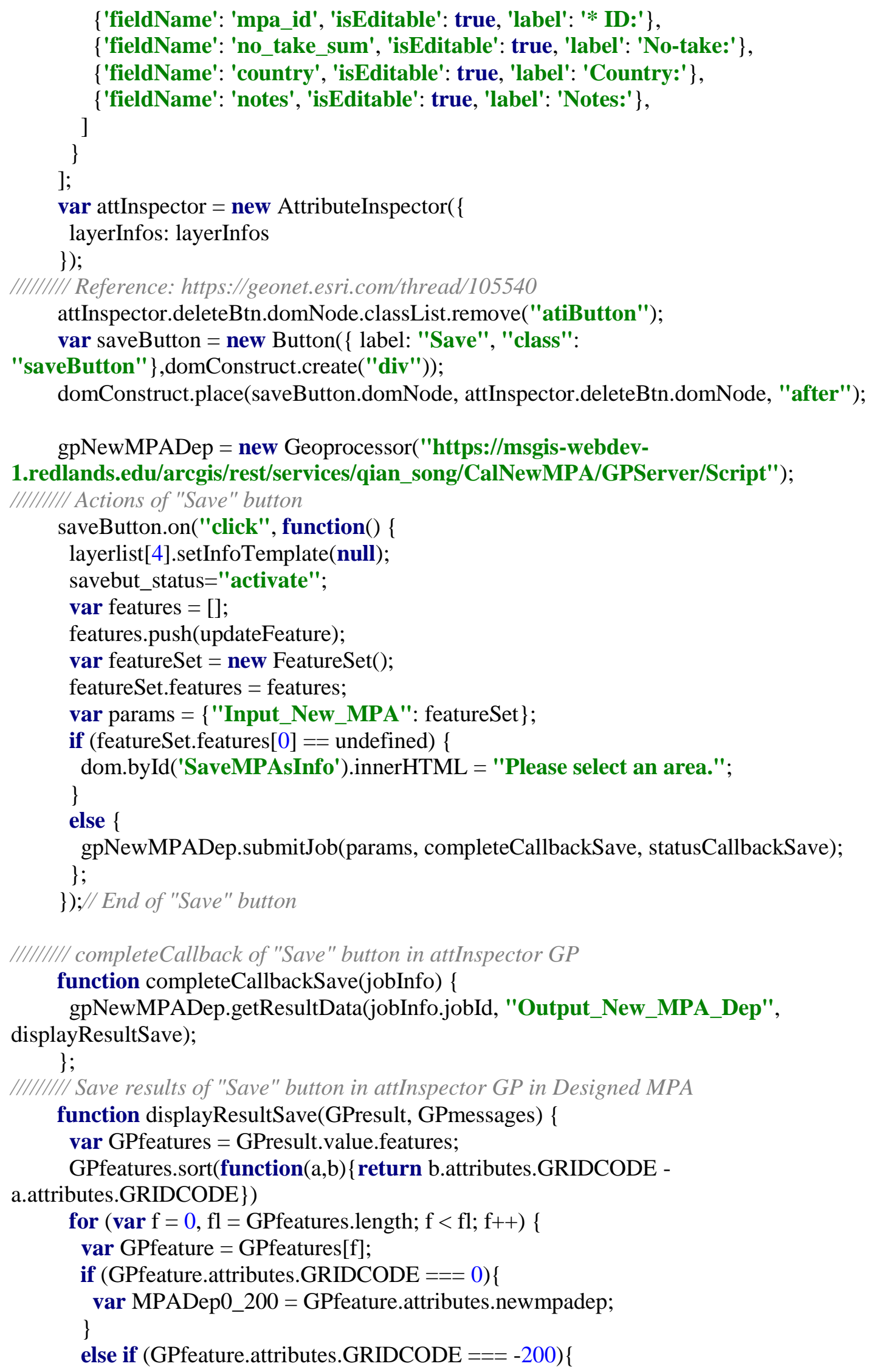




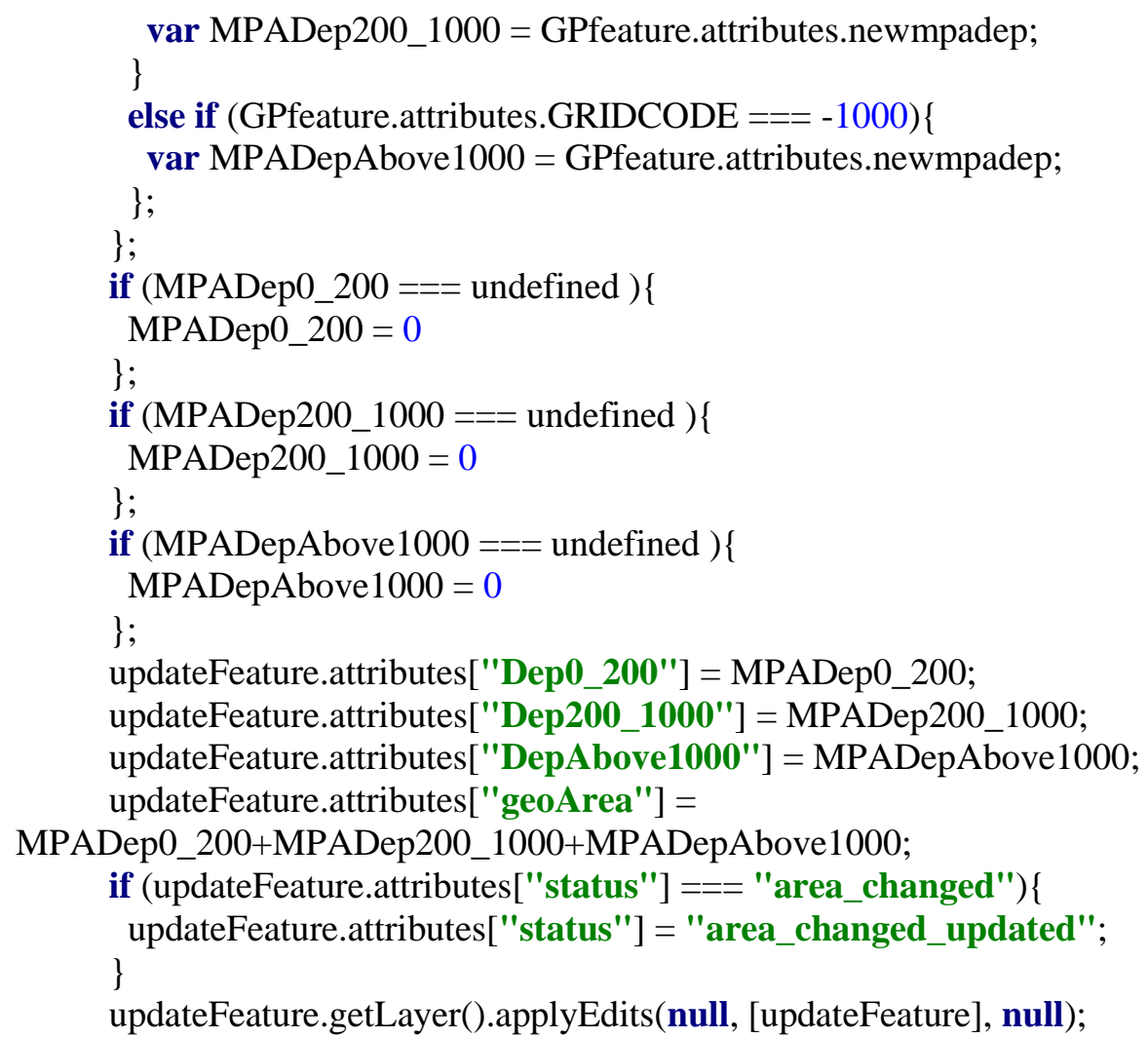




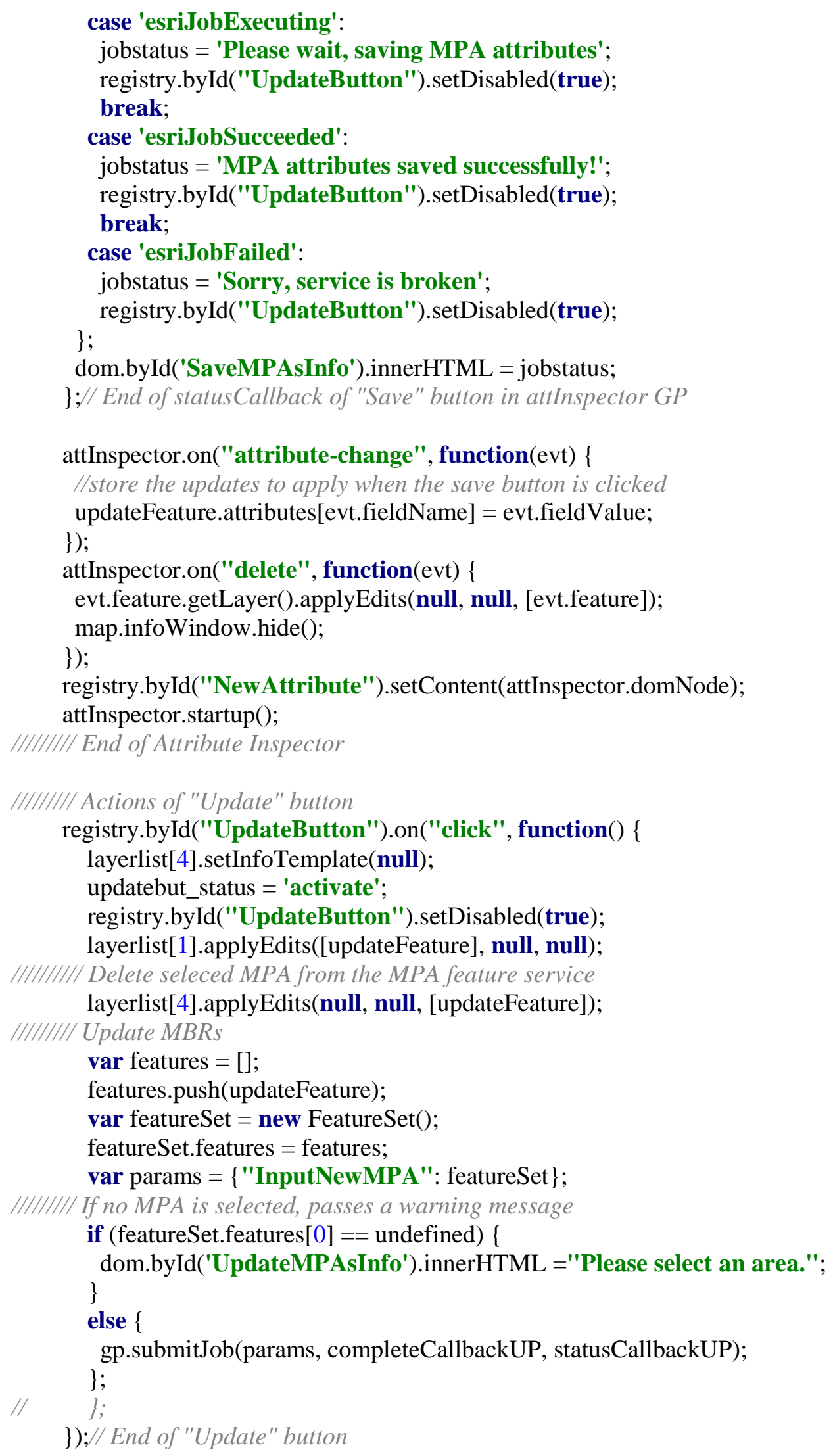




\section{I/IIIIII completeCallback of Update MBR GP}

function completeCallbackUP(jobInfo) \{

gp.getResultData(jobInfo.jobId, "BRoutput", displayResultUP);

;

IIIIIIIII Defined lists to save the new protected area in depth zones

var NewProDep0_200Tuplist=[]

var NewProDep200_1000Tuplist=[]

var NewProDepAbove1000Tuplist=[]

var BRECOCODETuplist=[]

var resultslist $=[]$

var listlen

IIIIIIII/ Save results of "Update" button GP in MBR feature

function displayResultUP(GPresult, GPmessages) \{

NewProDep0_200Tuplist $=$ []

NewProDep200_1000Tuplist $=$ []

NewProDepAbove1000Tuplist = []

BRECOCODETuplist=[]

resultslist $=[]$

listlen $=0$

var GPfeatures = GPresult.value.features;

I/IIIIII Sort GPfeatures in ascending order

GPfeatures.sort(function(a,b)\{return a.attributes.ECO_CODE -

b.attributes.ECO_CODE\})

listlen=GPfeatures.length

var query1 = new Query ();

query1.returnGeometry = true;

query1.outFields =

["ECO_CODE","ProDep0_200",,"ProDep200_1000","ProDepAbove1000"];

for $(\operatorname{var} \mathrm{f}=0, \mathrm{fl}=$ GPfeatures.length; $\mathrm{f}<\mathrm{fl} ; \mathrm{f}++)\{$

var GPfeature = GPfeatures[f];

var BRECOCODETup = GPfeature.attributes.ECO_CODE

var BRNameT=GPfeature. attributes.ECOREGION;

BRECOCODETuplist.push(BRECOCODETup)

var ProDep0_200T = GPfeature.attributes.ProDep0_200;

var ProDep200_1000T = GPfeature. attributes.ProDep200_1000;

var ProDepAbove1000T = GPfeature. attributes.ProDepAbove1000;

if (GPfeature.attributes.NewProDep0_200 == 0 || GPfeature.attributes.NewProDep0_200

$==$ undefined $)\{$

var NewProDep0_200Tup = ProDep0_200T;

\}

else \{

NewProDep0_200Tup = GPfeature.attributes.NewProDep0_200; \};

NewProDep0_200Tuplist.push(NewProDep0_200Tup)

if (GPfeature.attributes.NewProDep200_1000 == $0 \|$

GPfeature.attributes.NewProDep200_1000 == undefined ) \{

var NewProDep200_1000Tup = ProDep200_1000T;

\}

else \{

NewProDep200_1000Tup = GPfeature.attributes.NewProDep200_1000; 


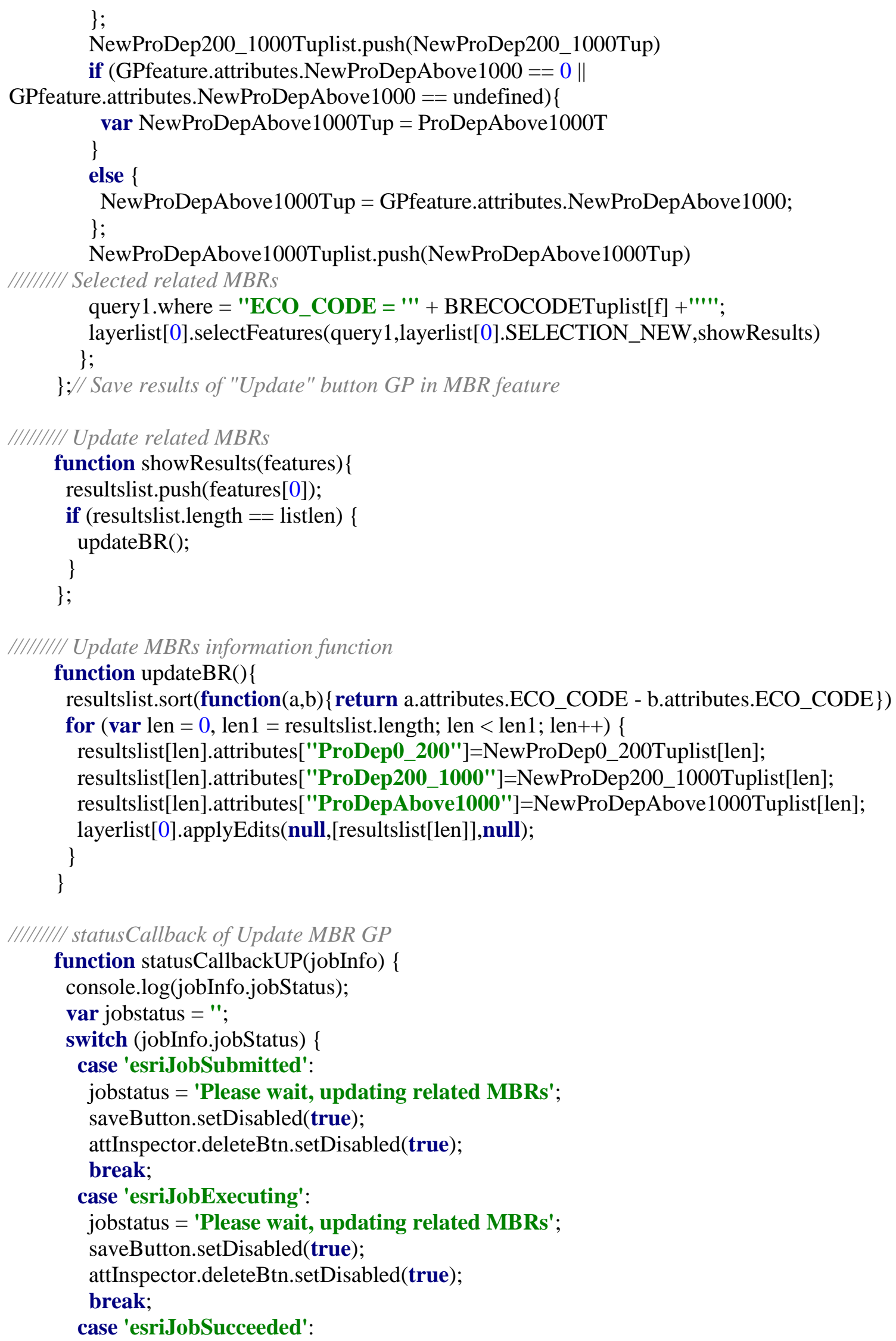




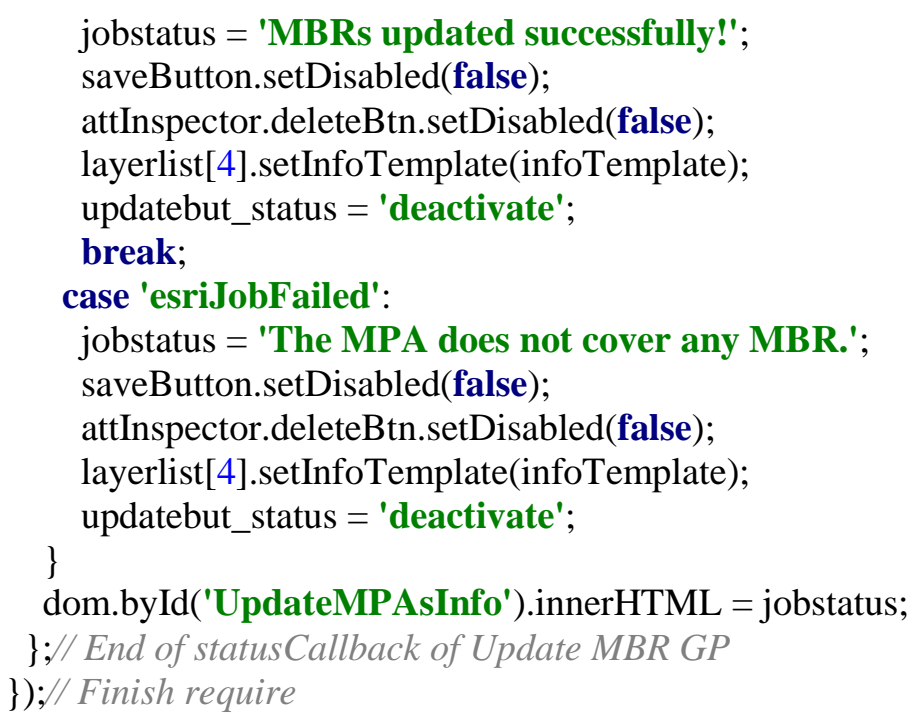




\section{Appendix C. CSS Code}

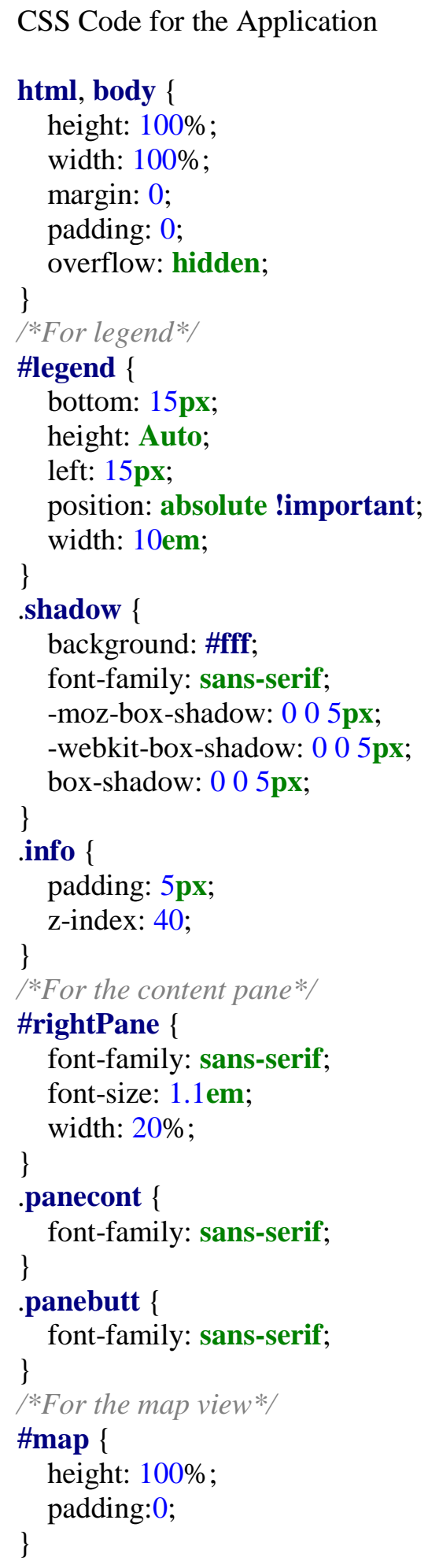

